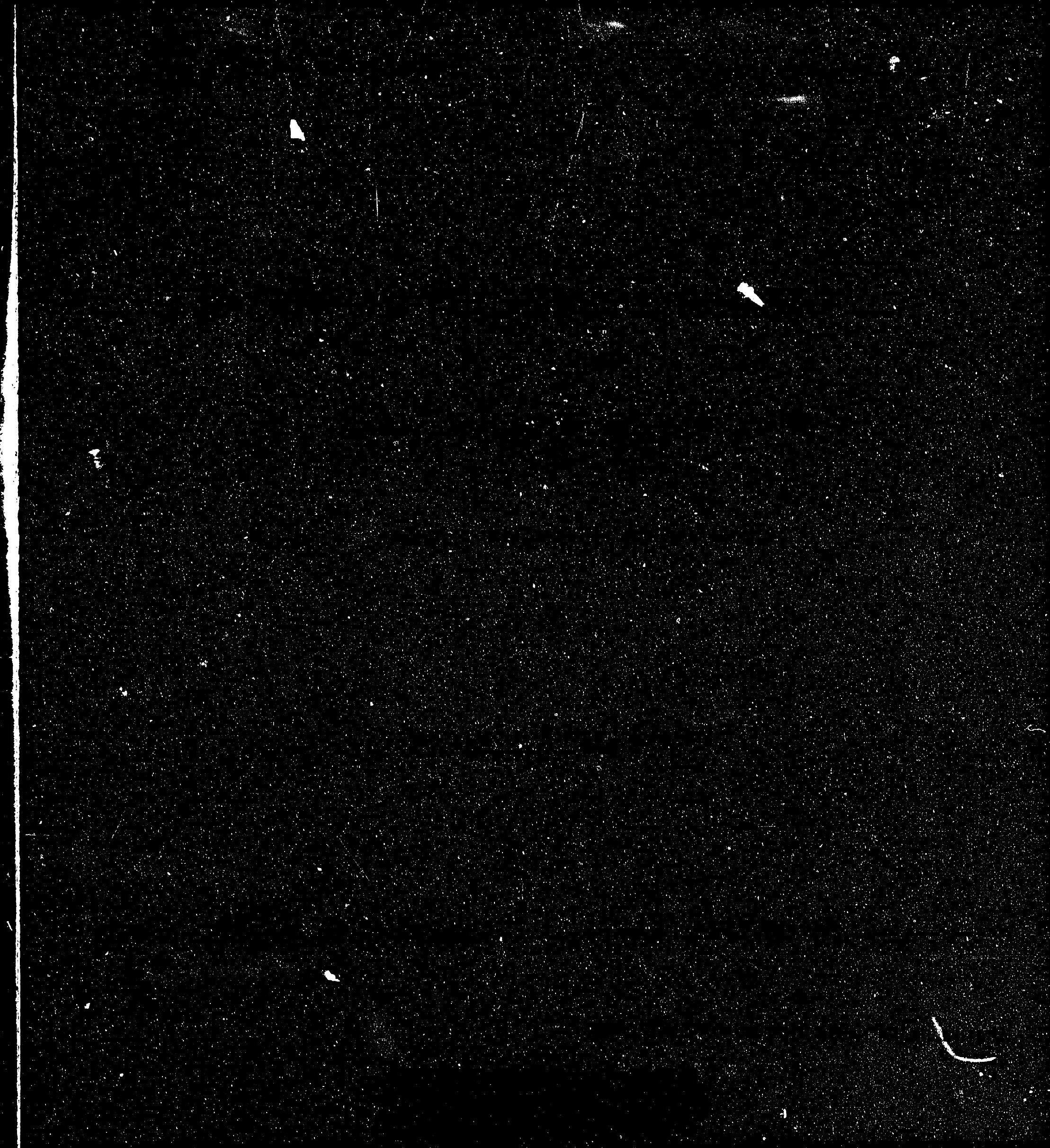


MEASURED THERMAL AND FAST NEUTRON FLUENCE RATES

ATR CYCLE $100-A$

$3 / 15 / 93$ Thru $4 / 22 / 93$

R. K. Murray and JW Rogers

This report contains the thermal $(2200 \mathrm{~m} / \mathrm{s})$ and fast (E>1MeV) neutron fluence rate data for ATR Cycle 100-A which were measured by the Radiation Measurements Laboratory (RML) as requested by the Power Reactor Programs (ATR Experiments) Radiation Measurements Work Order.

This report contains fluence rate values corresponding to the particular elevations (relative to the $80 \mathrm{ft}$. core elevation) where the measurements were taken. The data in this report consists of (1) a table of the ATR power history and distribution, (2) a hard copy listing of all thermal and fast neutron fluence rates, (3) plots of both the thermal and fast neutron fluence rates, and (4) a magnetic record ( 3.5 inch diskette) containing a listing of only the fast neutron fluence rates, their assigned elevations and proper header identification of all monitor positions contained herein.

The fluence rates reported are for the average power levels given in the table of power history and distribution.

All "H" holder monitor wires for this cycle are 54 inches long.

All "SR" holder monitor wires for this cycle are 55 inches long. This lengch allows measurement of the full core region and makes the first count elevation 24.73 inches above core midplane.

Due to the safety rod problems in the west lobe, "BR" holders were used in the $W-1,2,3$, and 4 positions. All "BR" holder monitor wires for this c.ycle are 56.25 inches long.

The distance from the end of the wires to the first count position was 4.25 inches for all wires counted from this cycle.

The results from the measurements in the $W-1,2,3,4$ mionitor positions indicate that the safety rod followers were rotated to a different azimuthal orientation relative to the normal orientation. The results indicate that the rotation was counterclockwise from their normal orientation. This is the same condition observed in cycle 99-B. 


\section{ON PRINTED PACIES}

1. CYCLE: The identification code given to a specific period of operation of the ATR for which the data in this report applies.

2. POSITION: The identification code given to a specific location in the ATR core area where neutron monitors were located and for which the data associated with the code apply. The letter code identifies the lobe and the number code identifies a monitor position.

3. MONITOR ID: The identification code given to a specific neutron monitor holder. The code is stamped on the holder. The letter code identifies the type of holder and the number is unique to allow tracking of the neutron monitors and their data.

4. EFPD: The acronym for, "Effective Full Power Days" which is the effective number of days the ATR operated at the average total core power level of the cycle. This value is obtained from the ATR power history and distribution.

5. AVERAGE MW: The average power level (in megawatts) at which the ATR lobe operated during the cycle. The values used for NW, NE, C, SW, and SE lobes are obtained from the ATR power history and distribution. The values used for the other lobes and core positions are determined as follows:

$$
\begin{aligned}
& N=(N E+N W+C) / 3 \\
& E=(N E+S E+C) / 3 \\
& S=(S E+S W+C) / 3 \\
& W=(N W+S W+C) / 3
\end{aligned}
$$

Al through $A 8=C$

$$
\begin{aligned}
A 9 & =N E \\
A 10 & =S E \\
A 11 & =S W \\
A 12 & =N W
\end{aligned}
$$
$B 1$ or $B 2=N E$
$B 3$ or $B 4=S E$
$B 5$ or $B 6=S W$
$B 7$ or $B 8=N W$

$$
\begin{aligned}
& B 9=N \\
& B 10=E \\
& B 11=S \\
& B 12=W
\end{aligned}
$$

6. DATE: The calendar dates corresponding to the outage and operating time of the cycle. These dates include the forced outage time associated with the cycle as well as the operating time. 
7. ELEVATION: The vertical position associated with the neutron fluence rate measurement value relative to the $80 \mathrm{ft}$. elevation of the ATR core. The elevation values are in inches and negative values are below the $80 \mathrm{ft}$. elevation (commonly referred to as centerline). The elevation values are established reference elevation points specified as follows:

\begin{tabular}{ccc} 
Holder Type & Reference Elevation & Drawing Numbers \\
\cline { 2 - 3 } & $77.948 \mathrm{ft}$. & 424852 \\
SR & $77.768 \mathrm{ft}$. & 417424 \\
BR-WEST POSITIONS & $81.771 \mathrm{ft}$. & 035561
\end{tabular}

Using the dimensions of the monitor holders, the location of the monitor wire in the holder, the length of the wire and the distance between the measurements along the wire an elevation value for each fluence rate value is determined. An evaluation of the elevation tolerances is listed as follows:

Holder Fabrication

Cutting the Monitor Wire to Length

Wire Alignment in the Holder

Mcunting the Wire to the Scanner

Scanning Control

Scanner Home Positioning

Stackup of the Safety Rod

Components (estimated)

Position of the Safety Rod Relative

to $80 \mathrm{ft}$. (core centerline)

during Operation

95\% Confidence Bound

Maximum Error
$S R$ \& BR

Holders

\pm 0.11 inch

\pm 0.01 inch

\pm 0.13 inch

\pm 0.13 inch

\pm 0.01 inch

\pm 0.06 inch

\pm 0.33 inch*

\pm 0.75 inch $\star \star$

\pm 0.78 inch

\pm 1.53 inch
H

$\frac{\text { Holders }}{ \pm 0.11 \text { inch }}$

\pm 0.01 inch

\pm 0.06 inch

\pm 0.13 inch

\pm 0.01 inch

\pm 0.06 inch

These elevation tolerances include only what are considered to be random errors. Systematic errors or biases may also occur due to information input. However, procedural controls are used to minimize this information input error.

*This estimate will be revised after an actual measurement is made.

$\star \star T h i s$ estimate does not include variations due to thermal and hydraulic effects. 
8. "THERMAL": The equivalent $2200 \mathrm{~m} / \mathrm{s}(0.025 \mathrm{eV})$ neutron fluence rate assuming a Maxwellian distribution for the thermalized neutrons at $20.40 \mathrm{C}$. The fluence rate is determined from the Co-59 $(n, \gamma) \mathrm{Co}-60$ reaction rate assuming a 37 barn $2200 \mathrm{~m} / \mathrm{s}$ cross section for the reaction. Corrections for the epithermal reaction rate are made based on cadmium ratio measurements at each position of the fluence rate measurement. All fluence rate values are in units of neutroris per sqcm per second.

9. "FAST":" The equivalent $>1$ MeV fission neutron energy fluence rate assuming a pure U-235 fission spectrum neutron energy distribution. The fluence rate is determined from the $\mathrm{Ni}-58(n, p) \mathrm{Co-58}$ reaction rate assuming a 0.092 barn fission spectrum averaged cross section for the reaction and a fission spectrum fraction of 0.692 for $>1 \mathrm{MeV}$ neutrons. Corrections for the burnout of the Co-58 isomers are made based on the measured $2200 \mathrm{~m} / \mathrm{s}$ fluence rate, the exposure duration and the burnout cross sections of the isomers [ 1650 barns ( 71 days), 1.7 $E+5$ barns ( 9.1 hours)] at each position of the fluence rate measurement.

10. CENTERLINE FLU. RATE: The neutron fluence rate at the elevation assignment nearest to the ATR core centerline elevation $(0.0)$.

11. AVG. MAX. FLU. RATE: The average of the highest three adjacent values.

12. MAX. FLU. RATE/MW (AVG.): The average maximum fluence rate divided $D$ the average power level (MW) of the lobe or position.

13. K FACTOR: The conversion factor determined from the neutron monitor activity for converting the net count rate at each elevation to the absolute reaction product $(60 \mathrm{Co}$ or $58 \mathrm{Co})$ radioactivity per unit mass of the monitor material $(\mathrm{d} / \mathrm{s} / \mathrm{mg})$.

14. RESONANCE CORRECTION: The fractional correction factor used to correct the $C_{0}-59(n, y) C_{0}-60$ reaction rate for the epithermal neutron response at the position where the neutron monitor was located during irradiation. This correction factor was determined from cobalt "cadmium ratio" measurements at the same spatial location for the monitor geometry and atom density.

15. C/L BURNOUT CORRECTION: The correction factor for the burnout (transmutation) of the reaction products ( $\left.\mathrm{Co}-60, \mathrm{Co}_{0}-58, \mathrm{Co}-58 \mathrm{~m}\right)$ of the neutron monitors during the cycle irradiation at the centerline fluence rate elevation. The burnout correction factors for other elevation assignments will vary (nonlinearly) depending on the "thermal" neutron fluence rates and cycle durations.

16. BACKGROUND (counts/sec.): The wire scanner detector background environment counting rate at the time the neutron monitor wires were scanned. 
17. CHECK SOURCE (counts/sec.)

Date Hour: The wire scanner detector check source count rate on the day the neutron monitor wires were scanned. The check source is a radioactive $\left({ }^{60} \mathrm{Co}\right)$ cobalt-aluminum wire mounted in a fixture which reproducibly positions it over the detector collimator. This check source is used to test counting functions (gain and stability) of the scanner counting system. The count rate is corrected for background and decay corrected to 05/01/88, 12:00.

\section{ON PLOTS}

18. REACTOR: Identification of the reactor with which the data are associated (normally ATR).

19. CYCLE POWER: The total megawatt days of power produced during the reactor cycle. This value is normally obtained from the ATR power history and distribution information supplied by PRP.

20. MATERIAL: The element name of the neutron monitor material. The "thermal" neutron monitor material is normally an alloy (COAl) of cobalt (CO) and aluminum (AI) where the weight percent is given preceding the percent (\%) symbol.

21. EXPERIMENT: The identification code given to the experiment located in the loop. This information is provided by PRP. 


\section{POWER HISTORY AND DISTRIBUTION}

CYCLE $100-A$

$3 / 15 / 93$ TO $4 / 22 / 93$

\begin{tabular}{|c|c|c|c|c|c|c|}
\hline & $\mathrm{NW}$ & $\mathrm{NE}$ & $c$ & SW & $\begin{array}{l}\text { TOTAL } \\
\text { SE }\end{array}$ & MWD \\
\hline MWd & 490 & 475 & 673 & 613 & 774 & 3024 \\
\hline $\begin{array}{l}\text { EFPD } \\
\text { Eff. Te. } \\
\text { PF }\end{array}$ & $\begin{array}{l}20.6 \\
11.14 \\
0.54\end{array}$ & $\begin{array}{l}10.79 \\
0.52\end{array}$ & $\begin{array}{l}15.29 \\
0.74\end{array}$ & $\begin{array}{l}13.93 \\
0.68\end{array}$ & $\begin{array}{l}17.59 \\
0.85\end{array}$ & \\
\hline $\begin{array}{l}\text { Average Power } \\
\text { Level in MW }\end{array}$ & 23.8 & 23.0 & 32.7 & 29.8 & 37.5 & \\
\hline
\end{tabular}

Due to significant down time during this cyle, the following power history was used for fluence rate determinations:

0.5 days $072 \mathrm{MW}=0.490$ P.F.

0.5 days $0144 \mathrm{MW}=0.981$ P.F.

4.4 days $145.5 \mathrm{MW}=0.991$ P.F.

2.4 days $0 \mathrm{MW}=0.0$ P.F.

11.6 days $0148 \mathrm{MW}=1.008$ P.F.

2.5 days $00 \mathrm{MW}=0.0 \mathrm{P} . \mathrm{F}$.

3.6 days $146 \mathrm{MW}=0.994$ P.F.

34 days $0 \mathrm{MW}=0.0$ P.F. 
CYCLE: $100-A$

EFPD: 20.6

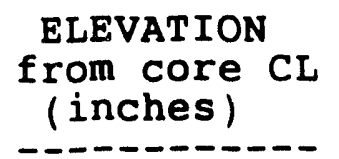

24.73

22.75

20.75

18.75

16.75

14.75

12.75

10.75

8.75

6.75

4.75

2.75

0.75

$-1.25$

$-3.25$

$-5.25$

$-7.25$

$-9.25$

$-11.25$

$-13.25$

$-15.25$

$-17.25$

$-19.25$

$-21.25$

$-23.25$

$-25.25$
POSITION : $\mathrm{N}-1$

AVERAGE MW: 26.5

"THERMAL"

$2200 \mathrm{~m} / \mathrm{s} \mathrm{CO}$

$(\mathrm{n} / \mathrm{cm} * \star 2 / \mathrm{sec})$

$1.49 E+13$

2. $52 \mathrm{E}+13$

$6.69 \mathrm{E}+13$

1. $12 \mathrm{E}+14$

$1.48 \mathrm{E}+14$

$1.70 \mathrm{E}+14$

1. $91 \mathrm{E}+14$

2.11E+14

$2.25 E+14$

$2.33 E+14$

$2.40 \mathrm{E}+14$

$2.46 \mathrm{E}+14$

$2.46 \mathrm{E}+14$

$2.47 \mathrm{E}+14$

$2.40 E+14$

$2.37 E+14$

2. $28 \mathrm{E}+14$

$2.21 E+14$

$2.10 E+14$

1. $85 \mathrm{E}+14$

1. $66 \mathrm{E}+14$

1. $44 \mathrm{E}+14$

1. $18 \mathrm{E}+14$

$9.48 \mathrm{E}+13$

$7 \cdot 38 \mathrm{E}+13$

$5 \cdot 28 E+13$
MONITOR ID: SR-463

DATE: $03 / 15 / 93$ to $04 / 22 / 93$

"FAST"

$>1 \mathrm{MeV}$ NI

$(\mathrm{n} / \mathrm{cm} * \star 2 / \mathrm{sec})$

1. $51 \mathrm{E}+13$

$2.42 E+13$

$3.99 \mathrm{E}+13$

$5.06 \mathrm{E}+13$

$6.24 \mathrm{E}+13$

$6.95 \mathrm{E}+13$

$7.81 \mathrm{E}+13$

$8.64 \mathrm{E}+13$

$9.10 \mathrm{E}+13$

$9.36 \mathrm{E}+13$

$9.12 \mathrm{E}+13$

$9.57 \mathrm{E}+13$

$9.89 \mathrm{E}+13$

$1.02 \mathrm{E}+14$

9. $69 \mathrm{E}+13$

$9.50 \mathrm{E}+13$

9. $43 \mathrm{E}+13$

$9.00 \mathrm{E}+13$

8. $72 E+13$

7. $91 \mathrm{E}+13$

7. $25 \mathrm{E}+13$

$6.26 E+13$

5. $25 E+13$

4. $13 E+13$

$2.73 E+13$

1. $48 \mathrm{E}+13$
CENTER LINE FLU. RATE

AVG. MAX. FLU. RATE

MAX . FLU. RATE/MW (AVG.)

K FACTOR

RESONANCE CORRECTION

C/L. BURNOUT CORRECTION

BACKGROUND (COUNTS/SEC.)

CHECK SOURCE (COUNTS/SEC.)

$05 / 14 / 9308: 51$
$2.46 \mathrm{E}+14$

$2.46 \mathrm{E}+14$

$9.30 \mathrm{E}+12$

292.48

0.76

1.001

1.37

1757.
9. $89 \mathrm{E}+13$

9. $91 E+13$

3. $74 \mathrm{E}+12$

3434.68

NA

1.776

1.37

1757 . 
CYCLE: 100-A

EFPD: 20.6

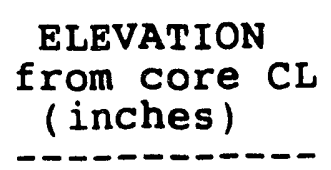

24.73

22.75

20.75

18.75

16.75

14.75

12.75

10.75

8.75

$6 .-5$

4. 5

$2 . .5$

0.75

$-1.25$

$-3.25$

$-5.25$

$-7.25$

$-9.25$

$-11.25$

$-13.25$

$-15.25$

$-17.25$

$-19.25$

$-21.25$

$-23.25$

$-25.25$
POSITION: $\mathrm{N}-3$

AVERAGE MW: 26.5

"THERMAL"

$2200 \mathrm{~m} / \mathrm{s} \mathrm{CO}$

$(\mathrm{n} / \mathrm{cm} * \star 2 / \mathrm{sec})$

$1.71 \mathrm{E}+13$

$2.73 E+13$

$6.92 \mathrm{E}+13$

$1.14 \mathrm{E}+14$

$1.54 \mathrm{E}+14$

$1.78 \mathrm{E}+14$

$2.00 \mathrm{E}+14$

$2.14 \mathrm{E}+14$

$2.19 \mathrm{E}+14$

$2.31 \mathrm{E}+14$

$2.37 \mathrm{E}+14$

$2.40 \mathrm{E}+14$

$2.40 \mathrm{E}+14$

2 . $1 \mathrm{E}+14$

2. $39 \mathrm{E}+14$

2. $32 \mathrm{E}+14$

$2.27 \mathrm{E}+14$

$2.17 \mathrm{E}+14$

$2.07 E+14$

1. $84 \mathrm{E}+14$

$1.64 \mathrm{E}+14$

$1.43 \mathrm{E}+14$

$1.18 \mathrm{E}+14$

9. $52 \mathrm{E}+13$

$7.88 \mathrm{E}+13$

$6.26 \mathrm{E}+13$
MONITOR ID: SR-464

DATE: $03 / 15 / 93$ to $04 / 22 / 93$

"FAST"

$>1 \mathrm{MeV}$ NI

$(\mathrm{n} / \mathrm{cm} * * 2 / \mathrm{sec})$

$2.66 \mathrm{E}+1 j$

$3.93 E+13$

$6.57 \mathrm{E}+13$

8. $36 \mathrm{E}+13$

$1.01 \mathrm{E}+14$

$1.13 \mathrm{E}+14$

$1.23 \mathrm{z}+14$

$1.28 \mathrm{E}+14$

$1.31 \mathrm{E}+14$

$1.38 \mathrm{E}+14$

$1.4 \mathrm{CE}+14$

$1.39 E+14$

1. $39 E+14$

1. $38 \mathrm{E}+14$

1. $37 \mathrm{E}+14$

1. $35 \mathrm{E}+14$

1. $31 \mathrm{E}+14$

1. $31 \mathrm{E}+14$

1. $17 \mathrm{E}+14$

1. $18 \mathrm{E}+14$

1. $09 \mathrm{E}+14$

9. $38 \mathrm{E}+13$

$7.68 \mathrm{E}+13$

$5.85 \mathrm{E}+13$

$3.54 \mathrm{E}+13$

1. $83 \mathrm{E}+13$
CENTER LINE FLU. RATE

AVG • MAX . FLU . RATE

MAX. FLU. RATE/MW (AVG.)

K FACTOR

RESONANCE CORRECTION

C/L. BURNOUT CORRECTION

BACKGROUND (COUNTS/SEC.)

CHECK SOURCB (COUNTS/SEC.) 05/13/93 08:56
$2.40 \mathrm{E}+14$

$2.40 \mathrm{E}+14$

$9.07 \mathrm{E}+12$

291.71

0.76

1.001

1.01

1764.
1. $39 E+14$

1. $39 E+14$

$5.26 \mathrm{E}+12$

3551.84

NA

1.757

1.01

1764 . 
CYCLE: $1.00-\mathrm{A}$

EFPD: $\quad 20.6$

ELEVATION
from core CL
(inches)

24.73

22.75

20.75

18.75

16.75

14.75

12.75

10.75

8.75

6.75

4.75

2.75

0.75

$-1.25$

$-3.25$

$-5.25$

$-7.25$

$-9.25$

$-11.25$

$-13.25$

$-15.25$

$-17.25$

$-19.25$

$-21.25$

$-23.25$

$-25.25$
POSITION: $\mathrm{N}-4$

AVERAGE MW: 26.5

"THERMAL"

$2200 \mathrm{~m} / \mathrm{s} \mathrm{CO}$

$(\mathrm{n} / \mathrm{cm} * \star 2 / \mathrm{sec})$

1. $38 \mathrm{E}+13$

2. $53 \mathrm{E}+13$

$7.06 \mathrm{E}+13$

1. $17 \mathrm{E}+14$

1. $55 \mathrm{E}+14$

1. $79 \mathrm{E}+14$

$2.02 \mathrm{E}+14$

$2.22 \mathrm{E}+14$

$2.29 E+14$

$2.37 E+14$

$2.44 \mathrm{E}+14$

$2.49 \mathrm{E}+14$

$2.51 E+14$

$2.49 \mathrm{E}+14$

$2.49 E+14$

$2.42 \mathrm{E}+14$

$2.31 \mathrm{E}+14$

2. $29 E+14$

$2.12 E+14$

$1.90 \mathrm{E}+14$

$1.72 \mathrm{E}+14$

1. $52 \mathrm{E}+14$

1. $25 \mathrm{E}+14$

9.57E+13

$7.11 \mathrm{E}+13$

4. $80 E+13$
MONITOR ID: SR-465

DATE : $03 / 15 / 93$ to $04 / 22 / 93$

"FAST"

$>1 \mathrm{MeV} N \mathrm{NI}$

$(\mathrm{n} / \mathrm{cm} \star \star 2 / \mathrm{sec})$

1. $34 \mathrm{E}+13$

$2.08 \mathrm{E}+13$

$2.95 E+13$

$3.76 \mathrm{E}+13$

4. $50 \mathrm{E}+13$

$5.18 \mathrm{E}+13$

$5.67 E+13$

$6.15 \mathrm{E}+13$

$6.37 \mathrm{E}+13$

$6.60 E+13$

$6.61 \mathrm{E}+13$

$6.73 E+13$

$6.75 \mathrm{E}+13$

$6.59 E+13$

$6.35 \mathrm{E}+13$

$6.62 \mathrm{E}+13$

$6.35 \mathrm{E}+13$

$6.00 \mathrm{E}+13$

$5.83 E+13$

$5.28 \mathrm{E}+13$

$4.74 \mathrm{E}+13$

$4.33 E+13$

3. $59 \mathrm{E}+13$

$2.71 \mathrm{E}+13$

$1.93 \mathrm{E}+13$

1. $13 \mathrm{E}+13$
CENTER LINE FLU. RATE

AVG. MAX. FLU. RATE

MAX . FLU. RATE/MW (AVG.)

$\mathrm{K}$ FACTOR

RESONANCE CORRECTION

C $/$ L. BURNOUT CORRECTION

BACKGROUND (COUNTS/SEC.)

CHECK SOURCE (COUNTS/SEC.)

$05 / 12 / 9306: 55$

\section{$2.51 E+14$}

$2.50 \mathrm{E}+14$

$9.43 \mathrm{E}+12$

293.50

0.81

1.001

1.36

1761 .
$6.75 \mathrm{E}+13$

$6.70 \mathrm{E}+13$

$2.53 E+12$

3679.69

NA

1.791

1.36

1761 . 
CYCLE: 100-A

EFPD: 20.6

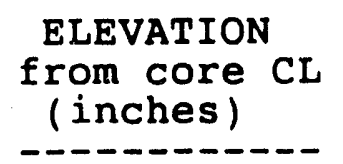

24.73

22.75

20.75

18.75

16.75

14.75

12.75

10.75

8.75

6.75

4.75

2.75

0.75

$-1.25$

$-3.25$

$-5.25$

$-7.25$

$-9.25$

$-11.25$

$-13.25$

$-15.25$

$-17.25$

$-19.25$

$-21.25$

$-23.25$

$-25.25$
POSITION: E-1

AVERAGE MW: 31.1

"THERMAL"

$2200 \mathrm{~m} / \mathrm{s} \mathrm{CO}$

$(\mathrm{n} / \mathrm{cm} * \star 2 / \mathrm{sec})$

$1.46 \mathrm{E}+13$

$2.70 E+13$

7. $65 \mathrm{E}+13$

$1.27 \mathrm{E}+14$

1. $74 \mathrm{E}+14$

$2.01 \mathrm{E}+14$

$2.35 E+14$

$2.54 E+14$

$2.72 E+14$

$2.85 E+14$

$2.90 E+14$

$2.99 E+14$

3. $03 E+14$

$3.03 E+14$

$2.99 \mathrm{E}+14$

$2.90 E+14$

$2.82 E+14$

$2.65 E+14$

$2.51 \mathrm{E}+14$

$2.30 E+14$

$1.96 \mathrm{E}+14$

$1.70 E+14$

$1.44 \mathrm{E}+14$

$1.14 E+14$

8. $30 E+13$

$5.52 E+13$
MONITOR ID: SR-466

DATE : $03 / 15 / 93$ to $04 / 22 / 93$

"FAST"

$>1 \mathrm{MeV} N \mathrm{NI}$

$(\mathrm{n} / \mathrm{cm} * \star 2 / \mathrm{sec})$

$1.50 \mathrm{E}+13$

$2.41 \mathrm{E}+13$

$3.44 \mathrm{E}+13$

$4.43 \mathrm{E}+13$

$5.47 \mathrm{E}+13$

$6.15 \mathrm{E}+13$

$6.87 \mathrm{E}+13$

$7.29 \mathrm{E}+13$

$7.80 \mathrm{E}+13$

8. $16 \mathrm{E}+13$

$8.36 \mathrm{E}+13$

8. $49 \mathrm{E}+13$

$8.67 \mathrm{E}+13$

$8.02 \mathrm{E}+13$

8. $29 \mathrm{E}+13$

8. $47 \mathrm{E}+13$

8. $24 \mathrm{E}+13$

$7.88 \mathrm{E}+13$

$7.68 \mathrm{E}+13$

$7.02 \mathrm{E}+13$

$6.47 \mathrm{E}+13$

$5.74 \mathrm{E}+13$

$4.79 \mathrm{E}+13$

$3.43 E+13$

$2.11 \mathrm{E}+13$

$1.41 \mathrm{E}+13$
CENTER LINE FLU. RATE

AVG. MAX. FLU. RATE

MAX. FLU. RATE/MW (AVG.)

$\mathrm{K}$ FACTOR

RESONANCE CORRECTION

C/L. BURNOUT CORRECTION

BACKGROUND (COUNTS/SEC.)

CHECK SOURCE (COUNTS/SEC.)

\section{$3.03 E+14$ \\ $3.01 E+14$ \\ $9.70 E+12$ \\ 293.70 \\ 0.81 \\ 1.001 \\ 1.39}

1764 .
8. $67 \mathrm{E}+13$

$8.51 \mathrm{E}+13$

2.74E+12

3741.75

NA

1.943

1.39

1764 . 


\begin{tabular}{|c|c|}
\hline $\begin{array}{l}\text { CYCLE: } \\
\text { EFPD: }\end{array}$ & $\begin{array}{l}\begin{array}{l}100-A \\
20.6\end{array} \\
\text { ELEVATION } \\
\text { from Core CI } \\
\text { (inches) } \\
- \\
24.73 \\
22.75 \\
20.75 \\
18.75 \\
16.75 \\
14.75 \\
12.75 \\
10.75 \\
8.75 \\
6.75 \\
4.75 \\
2.75 \\
0.75 \\
-1.25 \\
-3.25 \\
-5.25 \\
-7.25 \\
-9.25 \\
-11.25 \\
-13.25 \\
-15.25 \\
-17.25 \\
-19.25 \\
-21.25 \\
-23.25 \\
-25.25\end{array}$ \\
\hline
\end{tabular}

POSITION: E-2 AVERAGE MW: 31.1

"THERMAL" $2200 \mathrm{~m} / \mathrm{s} \mathrm{CO}$ $(\mathrm{n} / \mathrm{cm} * \star 2 / \mathrm{sec})$

$1.84 \mathrm{E}+13$

3. $12 \mathrm{E}+13$

8. $05 E+13$

1. $30 \mathrm{E}+14$

$1.72 \mathrm{E}+14$

$2.08 \mathrm{E}+14$

2. $33 E+14$

2. $54 \mathrm{E}+14$

$2.70 E+14$

2. $81 \mathrm{E}+14$

$2.90 E+14$

$2.98 E+14$

$2.98 \mathrm{E}+14$

$3.01 \mathrm{E}+14$

$2.98 \mathrm{E}+14$

$2.86 \mathrm{E}+14$

$2.82 E+14$

$2.61 \mathrm{E}+14$

$2.52 \mathrm{E}+14$

$2.25 \mathrm{E}+14$

$2.03 \mathrm{E}+14$

$1.71 \mathrm{E}+14$

1. $44 \mathrm{E}+14$

1. $16 \mathrm{E}+14$

$9.14 \mathrm{E}+13$

$6.60 E+13$
MONITOR ID: SR-467

DATE: $03 / 15 / 93$ to $04 / 22 / 93$

"FAST"

$>1 \mathrm{MeV} N \mathrm{NI}$

$(\mathrm{n} / \mathrm{cm} * * 2 / \mathrm{sec})$

$2.31 \mathrm{E}+13$

4. $02 E+13$

$6.15 E+13$

8. $01 \mathrm{E}+13$

9. $91 \mathrm{E}+13$

1. $15 \mathrm{E}+14$

1. $28 \mathrm{E}+14$

1. $37 \mathrm{E}+14$

1. $44 \mathrm{E}+14$

1. $52 \mathrm{E}+14$

1. $59 \mathrm{E}+14$

$1.64 \mathrm{E}+14$

1. $60 \mathrm{E}+14$

1. $62 \mathrm{E}+14$

1. $68 \mathrm{E}+14$

1. $63 \mathrm{E}+14$

$1.61 \mathrm{E}+14$

$1.50 \mathrm{E}+14$

1. $30 \mathrm{E}+14$

1. $26 \mathrm{E}+14$

$1.17 E+14$

1. $00 \mathrm{E}+14$

$7.85 \mathrm{E}+13$

$5.22 E+13$

$3.73 E+13$

2. $05 \mathrm{E}+13$
CENTER IINE FLU. RATE

AVG . MAX . FLU . RATE

MAX. FLU. RATE/MW (AVG.)

K FACTOR

RESONANCE CORRECTION

C/L. BURNOUT CORRECTION

BACKGROUND (COUNTS/SEC.)

CHECK SOURCE (COUNTS/SEC.) 05/13/93 08:56
$2.98 E+14$

$2.99 E+14$

9. $62 \mathrm{E}+12$

290.45

0.76

1.001

1.62

1764 .
1. $60 \mathrm{E}+14$

1. $64 \mathrm{E}+14$

5. $29 \mathrm{E}+12$

3563.02

NA

1.929

1.62

1764 . 
$\begin{array}{ll}\text { CYCLE: } & 100-A \\ \text { EFPD: } & 20.6\end{array}$

ELEVATION
from core CL
(inches)

24.73

22.75

20.75

18.75

16.75

14.75

12.75

10.75

8.75

6.75

4.75

2.75

0.75

$-1.25$

$-3.25$

$-5.25$

$-7.25$

$-9.25$

$-11.25$

$-13.25$

$-15.25$

$-17.25$

$-19.25$

$-21.25$

$-23.25$

$-25.25$
POSITION: E-3

AVERAGE MW: 31.1

"THERMAL"

$2200 \mathrm{~m} / \mathrm{s} \mathrm{CO}$

$(\mathrm{n} / \mathrm{cm} * \star 2 / \mathrm{sec})$

1. $88 \mathrm{E}+13$

$3.09 E+13$

$7.81 \mathrm{E}+13$

$1.26 \mathrm{E}+14$

$1.71 \mathrm{E}+14$

$2.02 \mathrm{E}+14$

$2.23 E+14$

$2.43 E+14$

$2.613+14$

$2.66 \mathrm{E}+14$

$2.80 E+14$

$2.90 \mathrm{E}+14$

$2.94 \mathrm{E}+14$

$2.90 E+14$

$2.89 E+14$

$2.81 \mathrm{E}+14$

$2.70 E+14$

$2.58 \mathrm{E}+14$

$2.45 E+14$

$2.21 E+14$

$1.98 \mathrm{E}+14$

$1.65 \mathrm{E}+14$

1. $39 \mathrm{E}+14$

$1.13 E+14$

$9.41 \mathrm{E}+13$

$7.54 \mathrm{E}+13$
MONITOR ID: SR-468

DATE: $03 / 15 / 93$ to $04 / 22 / 93$

"YAST"

$>1 \mathrm{MeV}$ NI

$(\mathrm{n} / \mathrm{cm} \star \star 2 / \mathrm{sec})$

3. $10 E+13$

4. $12 \mathrm{E}+13$

8.17E+13

1. $05 \mathrm{E}+14$

1. $27 \mathrm{E}+14$

$1.48 \mathrm{E}+14$

$1.58 \mathrm{E}+14$

$1.77 \mathrm{E}+14$

$1.83 E+14$

$1.86 \mathrm{E}+14$

1. $98 \mathrm{E}+14$

$1.98 \mathrm{E}+14$

$1.96 \mathrm{E}+14$

$2.02 E+14$

$2.02 E+14$

$2.01 E+14$

1. $93 \mathrm{E}+14$

1. $91 \mathrm{E}+14$

1.83E+14

$1.73 E+14$

1. $52 E+14$

1. $34 E+14$

$1.07 \mathrm{E}+14$

8. $31 \mathrm{E}+13$

4. $97 \mathrm{E}+13$

$2.52 E+13$
CENTER LINE FLU. RATE

AVG. MAX . FLU. RATE

MAX FLU. RATE/MW (AVG.)

K FACTOR

RESONANCE CORRECTION

C/L. BURNOUT CORRECTION

BACKGROUND (COUNTS/SEC.)

CHECK SOURCB (COUNTS/SEC.) 05/12/93 06:55
$2.94 \mathrm{E}+14$

$2.91 \mathrm{E}+14$

9.37E+12

287.15

0.76

1.001

1.26

1761 .
$1.96 \mathrm{E}+14$

$2.02 \mathrm{E}+14$

$6.50 \mathrm{E}+12$

3521.64

NA

1.917

1.26

1761 . 
CYCLE: 100-A

EFPD: 20.6

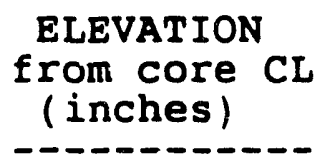

24.73

22.75

20.75

18.75

16.75

14.75

12.75

10.75

8.75

6.75

4.75

2.75

0.75

$-1.25$

$-3.25$

$-5.25$

$-7.25$

$-9.25$

$-11.25$

$-13.25$

$-15.25$

$-17.25$

$-19.25$

$-21.25$

$-23.25$

$-25.25$
POSITION: E-4

AVERAGE MW: 31.1

"THERMAL"

$2200 \mathrm{~m} / \mathrm{s} \mathrm{CO}$

$(\mathrm{n} / \mathrm{cm} * \star 2 / \mathrm{sec})$

$1.72 \mathrm{E}+13$

$2.75 E+13$

$6.98 E+13$

1. $12 \mathrm{E}+14$

1. $52 \mathrm{E}+14$

$1.79 E+14$

$2.05 E+14$

$2.21 \mathrm{E}+14$

$2.34 E+14$

$2.43 E+14$

$2.54 \mathrm{E}+14$

$2.59 E+14$

$2.61 E+14$

$2.65 E+14$

$2.57 E+14$

$2.48 \mathrm{E}+14$

$2.39 E+14$

$2.28 E+14$

2. $14 E+14$

1. $97 \mathrm{E}+14$

$1.69 \mathrm{E}+14$

1. $45 \mathrm{E}+14$

1. $24 \mathrm{E}+14$

$9.87 \mathrm{E}+13$

8. $02 E+13$

$6.38 E+13$
MONITOR ID: SR-469

DATE : $03 / 15 / 93$ to $04 / 22 / 33$

"FAST"

$>1 \mathrm{MeV}$ NI

$(\mathrm{n} / \mathrm{cm} \star \star 2 / \mathrm{sec})$

$2.72 \mathrm{E}+13$

4. $19 E+13$

$6.77 \mathrm{E}+13$

8. $47 \mathrm{E}+13$

$1.07 \mathrm{E}+14$

$1.17 E+14$

1. $33 \mathrm{E}+14$

$1.43 \mathrm{E}+14$

1. $50 \mathrm{E}+14$

1. $49 E+14$

$1.61 \mathrm{E}+14$

$1.62 \mathrm{E}+14$

$1.56 \mathrm{E}+14$

$1.63 \mathrm{E}+14$

$1.55 \mathrm{E}+14$

$1.56 \mathrm{E}+14$

$1.55 \mathrm{E}+14$

$1.53 E+14$

$1.46 \mathrm{E}+14$

1. $35 E+14$

$1.17 \mathrm{E}+14$

$1.08 \mathrm{E}+14$

$9.08 \mathrm{E}+13$

$6.84 \mathrm{E}+13$

4. $11 \mathrm{E}+13$

$2.08 E+13$
CENTER LINE FLU. RATE

AVG. MAX. FLU. RATE

MAX. FLU. RATE/MW (AVG.)

K FACTOR

RESONANCE CORRECTION

C/L. BURNOUT CORRECTION

BACKGROUND (COUNTS/SEC .)

CHECK SOURCE (COUNTS/SEC.)

$05 / 18 / 9308: 37$
$2.61 E+14$

$2.62 \mathrm{E}+14$

$8.42 E+12$

290.13

0.76

1.001

1.95

1745 .
$1.56 \mathrm{E}+14$

1. $60 \mathrm{E}+14$

5. $16 \mathrm{E}+12$

3574.18

NA

1.819

1.95

1745. 
CYCLE: 100-A

EFPD: 20.6

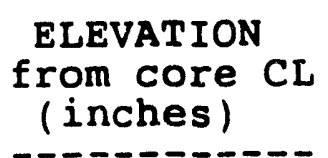

24.73

22.75

20.75

18.75

16.75

14.75

12.75

10.75

8.75

6.75

4.75

2.75

0.75

$-1.25$

$-3.25$

$-5.25$

$-7.25$

$-9.25$

$-11.25$

$-13.25$

$-15.25$

$-17.25$

$-19.25$

$-21.25$

$-23.25$

$-25.25$
POSITION: SE-1

AVERAGE MW: 37.5

"THERMAL"

$2200 \mathrm{~m} / \mathrm{s} \mathrm{CO}$

$(\mathrm{n} / \mathrm{cm} * \star 2 / \mathrm{sec})$

1. $44 \mathrm{E}+13$

$2.52 \mathrm{E}+13$

7. $00 \mathrm{E}+13$

1. $17 \mathrm{E}+14$

1. $58 \mathrm{E}+14$

$1.83 E+14$

$2.20 E+14$

$2.47 E+14$

$2.51 \mathrm{E}+14$

$2.61 E+14$

$2.70 \mathrm{E}+14$

$2.88 E+14$

$2.97 E+14$

$2.97 E+14$

3. $06 \mathrm{E}+14$

3. $18 \mathrm{E}+14$

3. $10 E+14$

3. $01 E+14$

$2.83 E+14$

$2.58 \mathrm{E}+14$

$2.28 \mathrm{E}+14$

$1.85 \mathrm{E}+14$

$1.55 E+14$

$1.20 \mathrm{E}+14$

9. $01 \mathrm{E}+13$

$7.21 E+13$
MONITOR ID: $S R-470$

DATE: $03 / 15 / 93$ to $04 / 22 / 93$

"FAST"

$>1 \mathrm{MeV}$ NI

$(\mathrm{n} / \mathrm{cm} * \star 2 / \mathrm{sec})$

\section{$2.73 E+13$}

$4.64 \mathrm{E}+13$

$7.75 \mathrm{E}+13$

$1.03 \mathrm{E}+14$

$1.29 \mathrm{E}+14$

1. $50 \mathrm{E}+14$

1. $74 \mathrm{E}+14$

1. $90 \mathrm{E}+14$

$2.02 \mathrm{E}+14$

$2.18 \mathrm{E}+14$

$2.05 E+14$

$2.24 \mathrm{E}+14$

$2.27 E+14$

$2.31 E+14$

2. $27 E+14$

$2.22 E+14$

$2.14 \mathrm{E}+14$

$2.04 E+14$

$1.96 \mathrm{E}+14$

$1.73 E+14$

$1.55 E+14$

1. $34 \mathrm{E}+14$

1. $09 \mathrm{E}+14$

8. $15 E+13$

4. $70 \mathrm{E}+13$

$2.57 E+13$
CENTER LINE FLU. RATE

AVG . MAX . FLU . RATE

MAX. 'FLU. RATE/MW (AVG.)

K FACTOR

RESONANCE CORRECTION

C/L. BURNOUT CORRECTION

BACKGROUND (COUNTS/SEC.)

CHECK SOURCE (COUNTS/SEC.) $05 / 12 / 9306: 55$

\section{2. $97 \mathrm{E}+14$}

3. $11 \mathrm{E}+14$

$8 \cdot 30 \mathrm{E}+12$

286.32

0.76

1.001

1.43

1761.
$2.27 \mathrm{E}+14$

$2.28 \mathrm{E}+14$

$6.08 E+12$

3571.82

NA

1.925

1.43

1761 . 
ATR NEUTRON MONITOR RESULTS

$\begin{array}{ll}\text { CYCLE: } & 100-A \\ \text { EFPD: } & 20.6\end{array}$

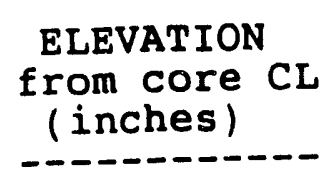

24.73

22.75

20.75

18.75

16.75

14.75

12.75

10.75

8.75

6.75

4.75

2.75

0.75

$-1.25$

$-3.25$

$-5.25$

$-7.25$

$-9.25$

$-11.25$

$-13.25$

$-15.25$

$-17.25$

$-19.25$

$-21.25$

$-23.25$

$-25.25$
POSITION: SE-2

AVERAGE MW: 37.5

"THERMAL"

$2200 \mathrm{~m} / \mathrm{s} \mathrm{CO}$

$(\mathrm{n} / \mathrm{cm} \star \star 2 / \mathrm{sec})$

1. $52 \mathrm{E}+13$

$2.49 E+13$

$6.98 \mathrm{E}+13$

1. $16 \mathrm{E}+14$

1. $60 \mathrm{E}+14$

$1.96 \mathrm{E}+16$

$2.27 E+14$

2. $57 \mathrm{E}+14$

$2.59 E+14$

$2.68 \mathrm{E}+14$

$2.86 \mathrm{E}+14$

3. $01 \mathrm{E}+14$

$3.07 \mathrm{E}+14$

3. $13 E+14$

3. $19 \mathrm{E}+14$

3. $28 E+14$

3. $26 E+14$

$3.13 E+14$

$2.95 \mathrm{E}+14$

$2.70 E+14$

2. $36 \mathrm{E}+14$

$2.02 E+14$

$1.61 \mathrm{E}+14$

1. $24 \mathrm{E}+14$

$9.46 \mathrm{E}+13$

$7.52 \mathrm{E}+13$
MONITOR ID: SR-471

DATE: $03 / 15 / 93$ to $04 / 22 / 93$

"FAST"

$>1 \mathrm{MeV}$ NI

$(\mathrm{n} / \mathrm{cm} \star \star 2 / \mathrm{sec})$

$2.48 \mathrm{E}+13$

$4.72 \mathrm{E}+13$

7. $31 \mathrm{E}+13$

$9.67 \mathrm{E}+13$

1. $23 \mathrm{E}+14$

$1.37 \mathrm{E}+14$

$1.59 \mathrm{E}+14$

$1.81 \mathrm{E}+14$

$1.93 \mathrm{E}+14$

$1.99 \mathrm{E}+14$

$2.03 E+14$

2. $05 E+14$

2. $20 \mathrm{E}+14$

$2.22 \mathrm{E}+14$

$2.19 E+14$

2. $12 E+14$

$2.00 \mathrm{E}+14$

$1.98 \mathrm{E}+14$

$1.80 \mathrm{E}+14$

$1.63 \mathrm{E}+14$

$1.49 \mathrm{E}+14$

1. $28 \mathrm{E}+14$

$1.06 \mathrm{E}+14$

$7.41 \mathrm{E}+13$

4. $20 \mathrm{E}+13$

$2.40 \mathrm{E}+13$
CENTER LINE FLU. RATE

AVG. MAX. FLU. RATE

MAX. FLU. RATE/MW (AVG.)

K FACTOR

RESONANCE CORRECTION

C/L. BURNOUT CORRECTION

BACKGROUND (COUNTS/SEC.)

CHECK SOURCE (COUNTS/SEC.) $05 / 14 / 9308: 51$
3. $07 \mathrm{E}+14$

$3.25 \mathrm{E}+14$

$8.66 E+12$

288.41

0.76

1.001

1.52

1757 .
$2.20 E+14$

$2.21 \mathrm{E}+14$

$5.88 \mathrm{E}+12$

3415.04

NA

1.956

1.52

1757. 
CYCLE: $100-A$

EFPD: 20.6

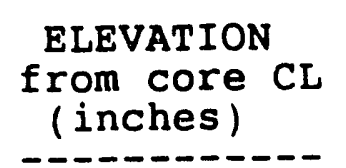

24.73

22.75

20.75

18.75

16.75

14.75

12.75

10.75

8.75

6.75

4.75

2.75

0.75

$-1.25$

$-3.25$

$-5.25$

$-7.25$

$-9.25$

$-11.25$

$-13.25$

$-15.25$

$-17.25$

$-19.25$

$-21.25$

$-23.25$

$-25.25$
POSITION: SE-3

AVERAGE MW: 37.5

"THERMAL"

$2200 \mathrm{~m} / \mathrm{s} \mathrm{CO}$

$(\mathrm{n} / \mathrm{cm} * \star 2 / \mathrm{sec})$

1. $58 \mathrm{E}+13$

$2.48 \mathrm{E}+13$

$7.07 \mathrm{E}+13$

1. $23 \mathrm{E}+14$

$1.67 \mathrm{E}+14$

$2.01 E+14$

2. $30 \mathrm{E}+14$

$2.60 E+14$

2. $69 \mathrm{E}+14$

$2.77 \mathrm{E}+14$

$2.91 \mathrm{E}+14$

3. $05 \mathrm{E}+14$

3. $11 \mathrm{E}+14$

3. $16 \mathrm{E}+14$

3. $20 \mathrm{E}+14$

$3.31 \mathrm{E}+14$

3. $32 E+14$

3. $19 \mathrm{E}+14$

$3.02 \mathrm{E}+14$

$2.76 \mathrm{E}+14$

$2.43 \mathrm{E}+14$

$2.12 \mathrm{E}+14$

$1.67 \mathrm{E}+14$

1. $33 \mathrm{E}+14$

$1.02 \mathrm{E}+14$

8. $58 \mathrm{E}+13$
MONITOR ID: SR-472

DATE: $03 / 15 / 93$ to $04 / 22 / 93$

"FAST"

$>1 \mathrm{MeV}$ NI

$(\mathrm{n} / \mathrm{cm} \star \star 2 / \mathrm{sec})$

$2.97 \mathrm{E}+13$

$5.44 \mathrm{E}+13$

$8.06 \mathrm{E}+13$

1. $09 \mathrm{E}+14$

1. $36 \mathrm{E}+14$

$1.58 \mathrm{E}+14$

$1.81 \mathrm{E}+14$

$1.99 \mathrm{E}+14$

$2.17 E+14$

$2.28 E+14$

$2.29 \mathrm{E}+14$

$2.43 E+14$

$2.48 \mathrm{E}+14$

$2.40 \mathrm{E}+14$

$2.40 \mathrm{E}+14$

$2.31 E+14$

$2.27 \mathrm{E}+14$

2. $09 \mathrm{E}+14$

$2.11 \mathrm{E}+14$

1.79E+14

$1.67 \mathrm{E}+14$

1. $47 \mathrm{E}+14$

1. $17 \mathrm{E}+14$

8. $70 \mathrm{E}+13$

4. $59 E+13$

2. $61 E+13$
CENTER LINE FLU. RATE

AVG. MAX. FLU. RATE

MAX . FLU. RATE/MW (AVG.)

K FACTOR

RESONANCE CORRECTION

C/L. BURNOUT CORRECTION

BACKGROUND (COUNTS/SEC .)

CHECK SOURCE (COUNTS/SEC.) $05 / 13 / 9308: 56$
3. $11 E+14$

3. $28 \mathrm{E}+14$

$8.73 \mathrm{E}+12$

296.28

0.76

1.001

1.19

1764 .
$2.48 \mathrm{E}+14$

$2.44 \mathrm{E}+14$

$6.50 E+12$

3555.16

NA

1.967

1.19

1764 . 
CYCLE: 100-A

EFPD: 20.6

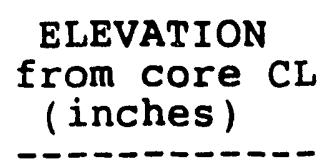

24.73

22.75

20.75

18.75

16.75

14.75

12.75

10.75

8.75

6.75

4.75

2.75

0.75

$-1.25$

$-3.25$

$-5.25$

$-7.25$

$-9.25$

$-11.25$

$-13.25$

$-15.25$

$-17.25$

$-19.25$

$-21.25$

$-23.25$

$-25.25$
POSITION: SE-4

AVERAGE MW: 37.5

"THERMAL"

$2200 \mathrm{~m} / \mathrm{s} \mathrm{CO}$

$(\mathrm{n} / \mathrm{cm} * \star 2 / \mathrm{sec})$

$1.69 \mathrm{E}+13$

$2.75 E+13$

$7.36 E+13$

1. $24 \mathrm{E}+14$

1. $66 \mathrm{E}+14$

$2.00 E+14$

$2.29 E+14$

$2.56 \mathrm{E}+14$

2. $60 \mathrm{E}+14$

$2.67 E+14$

$2.74 \mathrm{E}+14$

$2.83 E+14$

$2.93 E+14$

3. $01 \mathrm{E}+14$

$3.06 \mathrm{E}+14$

$3.11 \mathrm{E}+14$

$3.15 \mathrm{E}+14$

$3.06 \mathrm{E}+14$

$2.85 \mathrm{E}+14$

2. $64 \mathrm{E}+14$

$2.32 E+14$

$2.01 \mathrm{E}+14$

1. $60 \mathrm{E}+14$

1. $30 E+14$

$1.05 \mathrm{E}+14$

8. $35 E+13$
MONITOR ID: SR-473

DATE: $03 / 15 / 93$ to $04 / 22 / 93$

"FAST"

$>1 \mathrm{MeV} N \mathrm{NI}$

$(\mathrm{n} / \mathrm{cm} * 22 / \mathrm{sec})$

$2.95 E+13$

$4 \cdot 55 E+13$

$7.75 \mathrm{E}+13$

$1.02 \mathrm{E}+14$

1. $26 \mathrm{E}+14$

$1.51 \mathrm{E}+14$

$1.68 \mathrm{E}+14$

$1.87 \mathrm{E}+14$

$2.03 E+14$

$2.17 \mathrm{E}+14$

$2.21 \mathrm{E}+14$

$2.18 E+14$

$2.30 \mathrm{E}+14$

$2.24 \mathrm{E}+14$

$2.24 \mathrm{E}+14$

$2.11 \mathrm{E}+14$

2.17E+14

1. $95 \mathrm{E}+14$

$1.88 \mathrm{E}+14$

$1.72 \mathrm{E}+14$

$1.57 \mathrm{E}+14$

1. $39 \mathrm{E}+14$

$1.13 \mathrm{E}+14$

8. $42 \mathrm{E}+13$

$4.83 E+13$

$2.89 E+13$
CENTER IINE FLU. RATE

AVG. MAX. FLU. RATE

MAX . FLU. RATE/MW (AVG.)

$K$ FACTOR

RESONANCE CORRECTION

C/L . BURNOUT CORRECTION

BACKGROUND (COUNTS/SEC.)

CHECK SOURCE (COUNTS/SEC.)

$$
\text { 05/17/93 08:29 }
$$

$2.93 E+14$

3. $11 E+14$

$8.29 E+12$

292.28

0.76

1.001

1.53

1783 .
2. $30 \mathrm{E}+14$

2. $26 \mathrm{E}+14$

$6.03 E+12$

3487.97

NA

1.914

1.53

1783. 
CYCLE: $100-A$

EFPD: 20.6

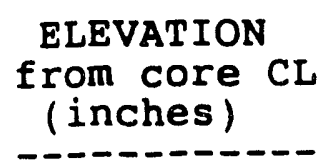

24.73

22.75

20.75

18.75

16.75

14.75

12.75

10.75

8.75

6.75

4.75

.75

75

$-1.25$

$-3.25$

$-5.25$

$-7.25$

$-9.25$

$-11.25$

$-13.25$

$-15.25$

$-17.25$

$-19.25$

$-21.25$

$-23.25$

$-25.25$
POSITION: $\mathrm{S}-1$

AVERAGE MW: 33.3

"THERMAL"

$2200 \mathrm{~m} / \mathrm{s} \mathrm{CO}$

$(\mathrm{n} / \mathrm{cm} * \star 2 / \mathrm{sec})$

$1.86 \mathrm{E}+13$

$2.82 \mathrm{E}+13$

$7.01 \mathrm{E}+13$

$1.24 \mathrm{E}+14$

$1.72 \mathrm{E}+14$

2. $10 E+14$

$2.39 \mathrm{E}+14$

$2.60 \mathrm{E}+14$

$2.78 E+14$

$2.93 E+14$

3. $00 \mathrm{E}+14$

3. $10 \mathrm{E}+14$

$3.19 \mathrm{E}+14$

3. $24 \mathrm{E}+14$

3. $20 \mathrm{E}+14$

3. $20 \mathrm{E}+14$

3. $26 \mathrm{E}+14$

3. $16 \mathrm{E}+14$

$2.99 E+14$

$2.78 \mathrm{E}+14$

$2.45 E+14$

$2.08 \mathrm{E}+14$

1. $68 \mathrm{E}+14$

1. $37 \mathrm{E}+14$

$1.15 E+14$

9. $80 \mathrm{E}+13$
MONITOR ID: $S R-474$

DATE : $03 / 15 / 93$ to $04 / 22 / 93$

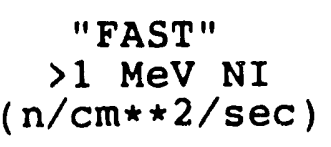

$2.77 E+13$

$4.91 \mathrm{E}+13$

$7.72 \mathrm{E}+13$

$9.93 E+13$

$1.25 \mathrm{E}+14$

$1.50 \mathrm{E}+14$

$1.65 \mathrm{E}+14$

$1.75 \mathrm{E}+14$

$1.95 \mathrm{E}+14$

$2.06 \mathrm{E}+14$

$2.10 \mathrm{E}+14$

$2.18 \mathrm{E}+14$

2. $09 \mathrm{E}+14$

2. $23 E+14$

$2.21 \mathrm{E}+14$

$2.18 \mathrm{E}+14$

2. $20 \mathrm{E}+14$

1. $97 \mathrm{E}+14$

$1.91 \mathrm{E}+14$

1. $77 \mathrm{E}+14$

1. $66 \mathrm{E}+14$

1. $43 E+14$

1. $17 \mathrm{E}+14$

8. $97 \mathrm{E}+13$

$5.73 E+13$

$2.97 \mathrm{E}+13$
CENTER LINE FLU. RATE

AVG. MAX . FLU. RATE

MAX. FLU. RATE/MW (AVG.)

K FACTOR

RESONANCE CORRECTION

C/L. BURNOUT CORRECTION

BACKGROUND (COUNTS/SEC .)

CHECK SOURCE (COUNTS/SEC.) 05/17/93 08:29

\section{3. $19 E+14$ \\ 3. $22 \mathrm{E}+14$ \\ $9.65 E+12$ \\ 296.16 \\ 0.76 \\ 1.001 \\ 1.71}

1783 .
$2.09 \mathrm{E}+14$

$2.21 E+14$

$6.62 \mathrm{E}+12$

3489.36

NA

1.992

1.71

1783 . 
CYCLE: 100-A

EFPD: $\quad 20.6$

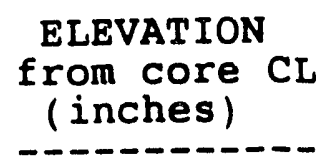

24.73

22.75

20.75

18.75

16.75

14.75

12.75

10.75

8.75

6.75

4.75

2.75

0.75

$-1.25$

$-3.25$

$-5.25$

$-7.25$

$-9.25$

$-11.25$

$-13.25$

$-15.25$

$-17.25$

$-19.25$

$-21.25$

$-23.25$

$-25.25$
POSITION: S-2

AVERAGE MW: 33.3

"THERMAL"

$2200 \mathrm{~m} / \mathrm{s} \mathrm{CO}$

$(\mathrm{n} / \mathrm{cm} \star \star 2 / \mathrm{sec})$

$1.45 \mathrm{E}+13$

2. $67 \mathrm{E}+13$

8. $12 \mathrm{E}+13$

1. $37 \mathrm{E}+14$

1. $90 \mathrm{E}+14$

$2.36 E \div 14$

$2.64 \mathrm{E}+14$

$2.84 \mathrm{E}+14$

$2.96 E+14$

$3.08 \mathrm{E}+14$

3. $24 \mathrm{E}+14$

$3.35 \mathrm{E}+14$

3. $49 \mathrm{E}+14$

$3.50 \mathrm{E}+14$

$3.45 \mathrm{E}+14$

$3.43 \mathrm{E}+14$

3. $49 \mathrm{E}+14$

3. $42 \mathrm{E}+14$

3. $20 \mathrm{E}+14$

2. $99 E+14$

$2.64 \mathrm{E}+14$

$2.22 \mathrm{E}+14$

$1.81 \mathrm{E}+14$

$1.44 \mathrm{E}+14$

$1.13 \mathrm{E}+14$

$7.52 \mathrm{E}+13$
MONITOR ID: SR-475

DATE : $03 / 15 / 93$ to $04 / 22 / 93$

"FAST"

$>1 \mathrm{MeV}$ NI

$(\mathrm{n} / \mathrm{cm} \star \star 2 / \mathrm{sec})$

$1.76 \mathrm{E}+13$

$2.47 E+13$

$3.95 \mathrm{E}+13$

$5.21 \mathrm{E}+13$

$6.41 \mathrm{E}+13$

$7.79 \mathrm{E}+13$

$8.46 \mathrm{E}+13$

8. $77 \mathrm{E}+13$

$9.19 \mathrm{E}+13$

$1.01 \mathrm{E}+14$

9.71E+13

$1.07 \mathrm{E}+14$

$1.06 \mathrm{E}+14$

$1.12 \mathrm{E}+14$

$1.14 \mathrm{E}+14$

$1.08 \mathrm{E}+14$

$9.71 \mathrm{E}+13$

$9.90 E+13$

9. $29 \mathrm{E}+13$

$9.06 \mathrm{E}+13$

$7.90 \mathrm{E}+13$

$6.51 \mathrm{E}+13$

$5.16 \mathrm{E}+13$

$4.22 \mathrm{E}+13$

$2.68 E+13$

1. $60 \mathrm{E}+13$
CENTER LINE FLU. RATE

AVG. MAX. FLU. RATE

MAX. FLU. RATE/MW (AVG.)

$\mathrm{K}$ FACTOR

RESONANCE CORRECTION

$C / L$. BURNOUT CORRECTION

BACKGROUND (COUNTS/SEC.)

CHECK SOURCE (COUNTS/SEC.) $05 / 18 / 9308: 37$
$3.49 \mathrm{E}+14$

$3.48 \mathrm{E}+14$

$1.04 \mathrm{E}+13$

296.97

0.81

1.001

1.57

1745 .
$1.06 \mathrm{E}+14$

1. $11 \mathrm{E}+14$

3. $34 \mathrm{E}+12$

3566.44

NA

2.081

1.57

1745 . 
CYCLE : 100-A

EFPD: 20.6

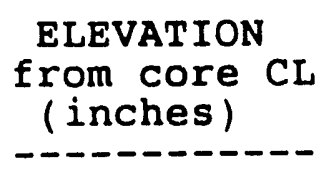

24.73

22.75

20.75

18.75

16.75

14.75

12.75

10.75

8.75

6.75

4.75

2.75

0.75

$-1.25$

$-3.25$

$-5.25$

$-7.25$

$-9.25$

$-11.25$

$-13.25$

$-15.25$

$-17.25$

$-19.25$

$-21.25$

$-23.25$

$-25.25$
POSITION: $\mathrm{S}-3$

AVERAGE MW: 33.3

"THERMAL"
$2200 \mathrm{~m} / \mathrm{s} \mathrm{CO}$
$\mathrm{n} / \mathrm{cm} * \star 2 / \mathrm{sec}$ )

$1.52 \mathrm{E}+13$

2. $53 \mathrm{E}+13$

$7.31 \mathrm{E}+13$

$1.29 \mathrm{E}+14$

$1.68 \mathrm{E}+14$

$2.14 \mathrm{E}+14$

$2.36 \mathrm{E}+14$

$2.52 \mathrm{E}+14$

$2.68 \mathrm{E}+14$

$2.89 \mathrm{E}+14$

$2.98 E+14$

$3.09 \mathrm{E}+14$

3. $12 \mathrm{E}+14$

$3.08 \mathrm{E}+14$

3. $11 \mathrm{E}+14$

3. $10 \mathrm{E}+14$

3. $15 \mathrm{E}+14$

3. $05 \mathrm{E}+14$

$2.83 E+14$

$2.63 \mathrm{E}+14$

$2.27 \mathrm{E}+14$

$1.84 \mathrm{E}+14$

$1.56 \mathrm{E}+14$

$1.28 \mathrm{E}+14$

$1.02 \mathrm{E}+14$

$7.77 \mathrm{E}+13$
MONITJR ID: SR-476

DATE - $03 / 15 / 93$ to $04 / 22 / 93$

$$
\begin{gathered}
\text { "F ST" } \\
>1: \text { eV NI } \\
(\mathrm{n} / \mathrm{cm} * * 2 / \mathrm{sec})
\end{gathered}
$$

$2 \cdot 25 \mathrm{E}+13$

$3.27 \mathrm{E}+13$

$5.24 \mathrm{E}+13$

$7.14 \mathrm{E}+13$

8. $71 E+13$

$1.04 \mathrm{E}+14$

$1.15 \mathrm{E}+14$

$1.21 \mathrm{E}+14$

$1.27 \mathrm{E}+14$

1. $32 \mathrm{E}+14$

1. $35 \mathrm{E}+14$

1. $39 \mathrm{E}+14$

$1.37 \mathrm{E}+14$

$1.38 \mathrm{E}+14$

$1.40 \mathrm{E}+14$

$1.29 E+14$

1. $29 \mathrm{E}+14$

1. $27 \mathrm{E}+14$

1. $21 \mathrm{E}+14$

$1.15 \mathrm{E}+14$

$1.04 \mathrm{E}+14$

$8.94 \mathrm{E}+13$

$7.43 \mathrm{E}+13$

$5.21 E+13$

$3.65 E+13$

$1.85 \mathrm{E}+13$
CENTER LINE FLU. RATE

AVG. MAX. FLU. RATE

MAX. FLU. RATE/MW (AVG.)

K FACTOR

RESONANCE CORRECTION

$\mathrm{C} / \mathrm{L}$. BURNOUT CORRECTION

BACKGROUND (COUNTS/SEC. )

CHECK SOURCE (COUNTS/SEC.) $05 / 14 / 9308: 51$
$3.12 \mathrm{E}+14$

3. $12 \mathrm{E}+14$

$9.35 \mathrm{E}+12$

289.48

0.76

1.001

1.46

1757 .
1. $37 \mathrm{E}+14$

1. $38 \mathrm{E}+14$

$4.15 E+12$

3513.92

NA

1.971

1.46

1757 . 
CYCLE : 100-A

EFPD: 20.6

ELEVATION
from core CL
(inches)

24.73

22.75

20.75

18.75

16.75

14.75

12.75

10.75

8.75

6.75

4.75

2.75

0.75

$-1.25$

$-3.25$

$-5.25$

$-7.25$

$-9.25$

$-11.25$

$-13.25$

$-15.25$

$-17.25$

$-19.25$

$-21.25$

$-23.25$

$-25.25$
POSITION: $\mathrm{S}-4$

AVERAGE MW: 33.3

"THERMAL"

$2200 \mathrm{~m} / \mathrm{s} \mathrm{CO}$

$(\mathrm{n} / \mathrm{cm} \star \star 2 / \mathrm{sec})$

$2.18 \mathrm{E}+13$

$3.62 \mathrm{E}+13$

$9.35 \mathrm{E}+13$

$1.44 \mathrm{E}+14$

$1.89 \mathrm{E}+14$

$2.26 \mathrm{E}+14$

$2.52 \mathrm{E}+14$

$2.73 E+14$

$2.88 \mathrm{E}+14$

$2.95 E+14$

3. $06 \mathrm{E}+14$

3. $17 E+14$

3. $22 \mathrm{E}+14$

3. $17 \mathrm{E}+14$

3. $19 \mathrm{E}+14$

3. $20 \mathrm{E}+14$

3. $22 \mathrm{E}+14$

$3.10 \mathrm{E}+14$

$2.91 E+14$

$2.66 E+14$

$2.31 E+14$

$1.87 \mathrm{E}+14$

1. $56 \mathrm{E}+14$

1. $27 \mathrm{E}+14$

$1.11 \mathrm{E}+14$

8. $94 \mathrm{E}+13$
MONITOR ID: $S R-477$

DATE : $03 / 15 / 93$ to $04 / 22 / 93$

\section{"FAST" \\ $>1 \mathrm{MeV}$ NI \\ $(\mathrm{n} / \mathrm{cm} \star \star 2 / \mathrm{sec})$}

$3.44 \mathrm{E}+13$

$4.56 \mathrm{E}+13$

8. $88 \mathrm{E}+13$

$1.11 \mathrm{E}+14$

1. $33 E+14$

$1.53 \mathrm{E}+14$

$1.75 \mathrm{E}+14$

$1.89 E+14$

$1.98 E+14$

$2.02 \mathrm{E}+14$

$2.12 \mathrm{E}+14$

$2.11 \mathrm{E}+14$

$2.14 \mathrm{E}+14$

$2.03 \mathrm{E}+14$

$2.06 \mathrm{E}+14$

$2.08 \mathrm{E}+14$

$2.05 \mathrm{E}+14$

1. $97 \mathrm{E}+14$

1. $87 \mathrm{E}+14$

1. $70 \mathrm{E}+14$

1. $55 \mathrm{E}+14$

1. $35 \mathrm{E}+14$

1. $09 \mathrm{E}+14$

8. $35 E+13$

5. $18 \mathrm{E}+13$

$2.48 E+13$
CENTER LINE FLU. RATE

AVG . MAX . FLU . RATE

MAX FLU . RATE/MW (AVG.)

K FACTOR

RESONANCE CORRECTION

C/L. BURNOUT CORRECTION

BACKGROUND (COUNTS/SEC.)

CHECK SOURCE (COUNTS/SEC.)

05/12/93 06:55

\section{3. $22 \mathrm{E}+14$ \\ 3. $20 \mathrm{E}+14$ \\ 9. $60 \mathrm{E}+12$ \\ 296.03 \\ 0.76 \\ 1.001 \\ 1.33}

1761 .
2. $14 \mathrm{E}+14$

2. $12 \mathrm{E}+14$

6. $36 \mathrm{E}+12$

3432.66

NA

2.001

1.33

1761 . 
$\begin{array}{ll}\text { CYCLE: } & 100-A \\ \text { EFPD: } & 20.6\end{array}$

ELEVATION
from core CL
(inches)

24.73

22.75

20.75

18.75

16.75

14.75

12.75

10.75

8.75

6.75

4.75

2.75

0.75

$-1.25$

$-3.25$

$-5.25$

$-7.25$

$-9.25$

$-11.25$

$-13.25$

$-15.25$

$-17.25$

$-19.25$

$-21.25$

$-23.25$

$-25.25$
POSITION: SW-1

AVERAGE MW: 29.8

"THERMAL"

$2200 \mathrm{~m} / \mathrm{s} \mathrm{CO}$

$(\mathrm{n} / \mathrm{cm} * * 2 / \mathrm{sec})$

1. $56 \mathrm{E}+13$

2. $53 E+13$

$6.37 \mathrm{E}+13$

1. $10 \mathrm{E}+14$

1. $52 \mathrm{E}+14$

$1.77 \mathrm{E}+14$

$2.07 E+14$

$2.26 \mathrm{E}+14$

$2.40 E+14$

$2.59 \mathrm{E}+14$

$2.76 \mathrm{E}+14$

$2.96 \mathrm{E}+14$

3. $03 E+14$

3. $00 E+14$

$2.97 \mathrm{E}+14$

$2.81 E+14$

$2.57 E+14$

$2.46 E+14$

$2.36 \mathrm{E}+14$

$2.25 E+14$

$2.10 \mathrm{E}+14$

$1.77 \mathrm{E}+14$

$1.57 \mathrm{E}+14$

$1.27 \mathrm{E}+14$

$1.05 \mathrm{E}+14$

$8.48 E+13$
MONITOR ID: $S R-478$

DATE : $03 / 15 / 93$ to $04 / 22 / 93$

"FAST"

$>1 \mathrm{MeV} N \mathrm{NI}$

$(\mathrm{n} / \mathrm{cm} \star \star 2 / \mathrm{sec})$

$2.63 \mathrm{E}+13$

$4.64 \mathrm{E}+13$

$6.69 \mathrm{E}+13$

8. $95 \mathrm{E}+13$

1. $11 \mathrm{E}+14$

$1.27 \mathrm{E}+14$

$1.45 \mathrm{E}+14$

$1.58 \mathrm{E}+14$

$1.68 \mathrm{E}+14$

$1.65 \mathrm{E}+14$

$1.68 \mathrm{E}+14$

$1.79 \mathrm{E}+14$

$1.74 \mathrm{E}+14$

$1.80 \mathrm{E}+14$

$1.77 \mathrm{E}+14$

$1.83 \mathrm{E}+14$

$1.73 \mathrm{E}+14$

$1.75 E+14$

$1.60 \mathrm{E}+14$

$1.47 \mathrm{E}+14$

1. $39 \mathrm{E}+14$

$1.17 E+14$

$1.01 E+14$

8. $00 \mathrm{E}+13$

$4.88 \mathrm{E}+13$

$2.76 \mathrm{E}+13$
CENTER LINE FLU. RATE

AVG. MAX . FLU. RATE

MAX. FLU. RATE/MW (AVG.)

$\mathrm{K}$ FACTOR

RESONANCE CORRECTION

C/L. BURNOUT CORRECTION

BACKGROUND (COUNTS/SEC. )

CHECK SOURCE (COUNTS/SEC.)

05/14/93 08:51

\section{$3.03 E+14$ \\ $3.00 \mathrm{E}+14$ \\ $1.01 \mathrm{E}+13$ \\ 291.25 \\ 0.76 \\ 1.001 \\ 1.26}

1757 .
1. $74 \mathrm{E}+14$

$1.80 \mathrm{E}+14$

$6.03 E+12$

3557.53

NA

1.945

1.26

1757 . 
CYCLE: 100-A

EFPD: 20.6

ELEVATION
from core CL
(inches)

POSITION: SW-2

AVERAGE MW: 29.8

"THERMAL"
$2200 \mathrm{~m} / \mathrm{s} \mathrm{CO}$
$(\mathrm{n} / \mathrm{cm} * \star 2 / \mathrm{sec})$

24.73

22.75

20.75

18.75

16.75

14.75

12.75

10.75

8.75

6.75

4.75

2.75

0.75

$-1.25$

$-3.25$

$-5.25$

$-7.25$

$-9.25$

$-11.25$

$-13.25$

$-15.25$

$-17.25$

$-19.25$

$-21.25$

$-23.25$

$-25.25$
1. $40 \mathrm{E}+13$

2. $44 \mathrm{E}+13$

$6.37 \mathrm{E}+13$

1. $06 \mathrm{E}+14$

1. $43 \mathrm{E}+14$

1. $70 \mathrm{E}+14$

$2.00 \mathrm{E}+14$

2. $17 \mathrm{E}+14$

$2.28 E+14$

$2.47 E+14$

$2.70 E+14$

$2.89 \mathrm{E}+14$

$2.91 \mathrm{E}+14$

$2.91 E+14$

$2.87 E+14$

$2.66 \mathrm{E}+14$

$2.46 \mathrm{E}+14$

2 . $32 \mathrm{E}+14$

$2.21 E+14$

2. $10 E+14$

1. $88 \mathrm{E}+14$

1. $66 \mathrm{E}+14$

1. $40 \mathrm{E}+14$

1. $10 \mathrm{E}+14$

8. $89 \mathrm{E}+13$

$6.89 E+13$
MONITOR ID: SR-479

DATE: $03 / 15 / 93$ to $04 / 22 / 93$

"FAST"

$>1 \mathrm{MeV} \mathrm{NI}$

$(\mathrm{n} / \mathrm{cm} \star \star 2 / \mathrm{sec})$

$2.48 \mathrm{E}+13$

$4.47 \mathrm{E}+13$

$6.78 \mathrm{E}+13$

$8.79 \mathrm{E}+13$

$1.08 \mathrm{E}+14$

1. $23 E+14$

1. $38 \mathrm{E}+14$

$1.50 \mathrm{E}+14$

$1.56 \mathrm{E}+14$

$1.63 \mathrm{E}+14$

$1.66 \mathrm{E}+14$

$1.68 \mathrm{E}+14$

$1.61 \mathrm{E}+14$

$1.74 \mathrm{E}+14$

$1.72 \mathrm{E}+14$

$1.71 \mathrm{E}+14$

$1.68 \mathrm{E}+14$

$1.61 \mathrm{E}+14$

1. $50 \mathrm{E}+14$

1. $39 E+14$

1. $25 \mathrm{E}+14$

$1.02 \mathrm{E}+14$

9. $01 \mathrm{E}+13$

$6.62 \mathrm{E}+13$

$3.82 \mathrm{E}+13$

$2.01 E+13$
CENTER LINE FLU. RATE

AVG . MAX - FLU . RATE

MAX. FLU. RATE/MW (AYG.)

K FACTOR

RESONANCE CORRECTION

C/L . BURNOUT CORRECTION

BACKGROUND (COUNTS/SEC .)

CHECK SOURCB (COUNTS/SEC.) 05/12/93 06:55
$2.91 \mathrm{E}+14$

$2.91 E+14$

$9.75 E+12$

295.88

0.76

1.001

1.32

1761 .
$1.61 \mathrm{E}+14$

$1.73 E+14$

$5.79 \mathrm{E}+12$

3532.64

NA

1.909

1.32

1761 . 


$$
\begin{array}{ll}
\text { CYCLE: } & 100-A \\
\text { EFPD: } & 20.6
\end{array}
$$

$$
\begin{aligned}
& \text { ELEVATION } \\
& \text { from core CL } \\
& \text { (inches) }
\end{aligned}
$$

24.73
22.75
20.75
18.75
16.75
14.75
12.75
10.75
8.75
6.75
4.75
2.75
0.75
-1.25
-3.25
-5.25
-7.25
-9.25
-11.25
-13.25
-15.25
-17.25
-19.25
-21.25
-23.25
-25.25

POSI ION: SW-3

AVER JE MW: 29.8

"THERMAL"

$2200 \mathrm{~m} / \mathrm{s} \mathrm{CO}$

$(\mathrm{n} / \mathrm{cm} \star 2 / \mathrm{sec})$

1. $26 \mathrm{E}+13$

$2.11 \mathrm{E}+13$

$5.56 \mathrm{E}+13$

$9.50 \mathrm{E}+13$

1. $29 \mathrm{E}+14$

$1.53 \mathrm{E}+14$

$1.73 E+14$

$1.98 \mathrm{E}+14$

$2 \cdot 16 \mathrm{E}+14$

$2.33 E+14$

$2.52 E+14$

$2.67 E+14$

$2.75 \mathrm{E}+14$

$2.77 \mathrm{E}+14$

$2.68 \mathrm{E}+14$

$2 \cdot 52 E+14$

$2.31 \mathrm{E}+14$

$2.13 E+14$

$2.05 E+14$

$1.96 \mathrm{E}+14$

$1.74 \mathrm{E}+14$

$1.53 \mathrm{E}+14$

1. $32 \mathrm{E}+14$

i. $03 E+14$

$8.15 E+13$

$6.46 \mathrm{E}+13$
MONITOR ID: SR-480

DATE: $03 / 15 / 93$ to $04 / 22 / 93$

\author{
"FAST" \\ $>1 \mathrm{MeV}$ NI \\ $(\mathrm{n} / \mathrm{cm} \star * 2 / \mathrm{sec})$
}

1. $98 \mathrm{E}+13$

$3.83 E+13$

$5.73 \mathrm{E}+13$

$7.98 \mathrm{E}+13$

$9.74 \mathrm{E}+13$

$1.14 \mathrm{E}+14$

1. $25 E+14$

1. $35 E+14$

$1.49 \mathrm{E}+14$

$1.46 \mathrm{E}+14$

$1.55 \mathrm{E}+14$

$1.6: 5+14$

$1.6 \mathrm{C} \mathrm{\Xi}+14$

$1.62 \mathrm{E}+14$

1. $50 \mathrm{E}+14$

$1.60 \mathrm{E}+14$

$1.55 \mathrm{E}+14$

1. $43 \mathrm{E}+14$

$1.35 \mathrm{E}+14$

1. $24 \mathrm{E}+14$

$1.19 \mathrm{E}+14$

$1.02 \mathrm{E}+14$

$8 \cdot 58 \mathrm{E}+13$

$6.27 \mathrm{E}+13$

4. $29 E+13$

$1.97 \mathrm{E}+13$
CENTER LINE FLU. RATE

AVG. MAX. FLU. RATE

MAX. 'FLU. RATE/MW (AVG.)

K FACTOR

RESONANCE CORRECTION

C/L. BURNOUT CORRECTION

BACKGROUND (COUNTS/SEC.)

CHECK SOURCE (COUNTS/SEC.) $05 / 18 / 9308: 37$
$2.75 \mathrm{E}+14$

$2.73 \mathrm{E}+14$

$9.16 \mathrm{E}+12$

288.86

0.76

1.001

1.62

1745 .
$1.60 \mathrm{E}+14$

$1.61 \mathrm{E}+14$

$5.40 \mathrm{E}+12$

3578.24

NA

1.860

1.62

1745 . 


\section{CYCLE: 100-A \\ EFPD: 20.6}

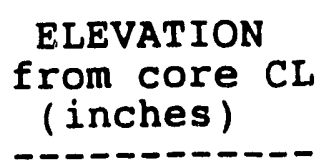

24.73

22.75

20.75

18.75

16.75

14.75

12.75

10.75

8.75

6.75

4.75

2.75

0.75

$-1.25$

$-3.25$

$-5.25$

$-7.25$

$-9.25$

$-11.25$

$-13.25$

$-15.25$

$-17.25$

$-19.25$

$-21.25$

$-23.25$

$-25.25$
POSITION: SW-4

AVERAGE MW: 29.8

"THERMAL"

$2200 \mathrm{~m} / \mathrm{s} \mathrm{CO}$

$(\mathrm{n} / \mathrm{cm} * \star 2 / \mathrm{sec})$

$1.52 \mathrm{E}+13$

$2.46 \mathrm{E}+13$

$6.56 \mathrm{E}+13$

$1.07 \mathrm{E}+14$

$1.45 \mathrm{E}+14$

1. $70 \mathrm{E}+14$

1. $90 \mathrm{E}+14$

$2.16 \mathrm{E}+14$

$2.29 E+14$

$2.52 \mathrm{E}+14$

$2.73 \mathrm{E}+14$

$2.83 \mathrm{E}+14$

$2.87 E+14$

$2.90 \mathrm{E}+14$

$2.83 E+14$

$2.62 E+14$

$2.41 \mathrm{E}+14$

$2.31 E+14$

$2.15 E+14$

$2.07 E+14$

$1.86 \mathrm{E}+14$

$1.63 \mathrm{E}+14$

$1.41 \mathrm{E}+14$

1. $13 \mathrm{E}+14$

9. $20 \mathrm{E}+13$

7. $26 \mathrm{E}+13$
MONITOR ID: SR-481

DATE : $03 / 15 / 93$ to $04 / 22 / 93$

"FAST"

$>1 \mathrm{MeV}$ NI

$(\mathrm{n} / \mathrm{cm} * \star 2 / \mathrm{sec})$

$2.80 \mathrm{E}+13$

$4.32 \mathrm{E}+13$

$7.33 E+13$

9. $40 \mathrm{E}+13$

$1.16 \mathrm{E}+14$

$1.31 \mathrm{E}+14$

$1.46 \mathrm{E}+14$

$1.61 \mathrm{E}+14$

$1.70 \mathrm{E}+14$

$1.75 \mathrm{E}+14$

$1.77 \mathrm{E}+14$

$1.76 \mathrm{E}+14$

$1.81 \mathrm{E}+14$

$1.83 \mathrm{E}+14$

$1.81 \mathrm{E}+14$

$1.77 \mathrm{E}+14$

$1.73 \mathrm{E}+14$

$1.65 \mathrm{E}+14$

$1.57 \mathrm{E}+14$

1. $49 \mathrm{E}+14$

$1.28 \mathrm{E}+14$

$1.15 E+14$

$9.85 \mathrm{E}+13$

$7.31 E+13$

$4.48 \mathrm{E}+13$

$1.97 \mathrm{E}+13$
CENTER LINE FLU. RATE

AVG. MAX. FLU. RATE

MAX. FLU. RATE/MW (AVG.)

K FACTOR

RESONANCE CORRECTION

C/L. BURNOUT CORRECTION

BACKGROUND (COUNTS/SEC.)

CHECK SOURCE (COUNTS/SEC.) 05/12/93 06:55
$2.87 \mathrm{E}+14$

$2.87 \mathrm{E}+14$

$9.62 \mathrm{E}+12$

295.65

0.76

1.001

1.39

1761 .
$1.81 \mathrm{E}+14$

$1.81 \mathrm{E}+14$

$6.09 \mathrm{E}+12$

3644.82

NA

1.897

1.39

1761 . 
CYCLE: 100-A

EFPD: 20.6

ELEVATION
from core CL
(inches)

20.50

19.50

17.50

15.50

13.50

11.50

9.50

7.50

5.50

3.50

1.50

$-0.50$

$-2.50$

$-4.50$

$-6.50$

$-8.50$

$-10.50$

$-12.50$

$-14.50$

$-16.50$

$-18.50$

$-20.50$

$-22.50$

$-24.50$

.26 .50

$-28.50$

$-30.48$
POSITION: $W-1$

AVERAGE MW: 28.8

"THERMAL"

$2200 \mathrm{~m} / \mathrm{s} \mathrm{CO}$

$(\mathrm{n} / \mathrm{cm} * * 2 / \mathrm{sec})$

$1.20 \mathrm{E}+14$

1. $34 \mathrm{E}+14$

1. $70 \mathrm{E}+14$

$2.13 \mathrm{E}+14$

$2.37 E+14$

$2.56 \mathrm{E}+14$

$2.72 \mathrm{E}+14$

$2.84 E+14$

$2.95 \mathrm{E}+14$

3. $10 \mathrm{E}+14$

3. $34 \mathrm{E}+14$

3. $26 \mathrm{E}+14$

3. $14 \mathrm{E}+14$

3. $08 \mathrm{E}+14$

$3.01 E+14$

$2.91 E+14$

$2.76 \mathrm{E}+14$

$2.56 \mathrm{E}+14$

$2.31 \mathrm{E}+14$

$1.92 E+14$

$1.63 E+14$

1. $34 \mathrm{E}+14$

$1.07 \mathrm{E}+14$

$9.24 \mathrm{E}+13$

5. $95 E+13$

3. $13 \mathrm{E}+13$

$1.41 \mathrm{E}+13$
MONITOR ID: BR-482

DATE : $03 / 15 / 93$ to $04 / 22 / 93$

"FAST"

$>1 \mathrm{MeV}$ NI

$(\mathrm{n} / \mathrm{cm} * \star 2 / \mathrm{sec})$

$8.42 E+13$

$9 \cdot 13 \mathrm{E}+13$

$1.15 \mathrm{E}+14$

1. $36 \mathrm{E}+14$

$1.46 \mathrm{E}+14$

$1.47 \mathrm{E}+14$

$1.61 \mathrm{E}+14$

$1.65 \mathrm{E}+14$

$1.72 \mathrm{E}+14$

$1.69 \mathrm{E}+14$

$1.67 \mathrm{E}+14$

$1.69 \mathrm{E}+14$

$1.71 \mathrm{E}+14$

$1.76 \mathrm{E}+14$

$1.76 \mathrm{E}+14$

$1.53 E+14$

$1.60 E+14$

$1.55 \mathrm{E}+14$

$1.46 \mathrm{E}+14$

1. $21 \mathrm{E}+14$

1. $10 \mathrm{E}+14$

$9.04 \mathrm{E}+13$

6. $64 \mathrm{E}+13$

3. $3 . E+13$

$1.48 \mathrm{E}+13$

$6.89 E+12$

$3.18 E+12$
CENTER LINE FLU. RATE

AVG. MAX. FIU, RATE

MAX. FLU. RATE/HW (AVG.)

K FACTOR

RESONANCE CORRECTION

C/L. BURNOUT CORRECTION

BACKGROUND (CCUNTS/SEC.)

CHECK SOURCE (COUNTS/SEC.) 05/19/93 08:33
$3.26 \mathrm{E}+14$

3. $24 \mathrm{E}+14$

$1.13 \mathrm{E}+13$

301.26

0.76

1.001

1.60

1770 .
$1.69 \mathrm{E}+14$

$1.75 \mathrm{E}+14$

$6.07 \mathrm{E}+12$

3621.40

NA

2.012

1.60

1770 . 
ATR NEUTRON MONITOR RESULTS

CYCLE: 100-A

EFPD: 20.6

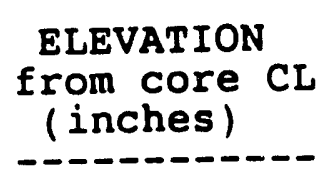

20.50

19.50

17.50

15.50

13.50

11.50

9.50

7.50

5.50

3.50

1.50

$-0.50$

$-2.50$

$-4.50$

$-6.50$

$-8.50$

$-10.50$

$-12.50$

$-14.50$

$-16.50$

$-18.50$

$-20.50$

$-22.50$

$-24.50$

$-26.50$

$-28.50$

$-30.48$
POSITION: $\mathrm{W}-2$

AVERAGE MW: 28.8

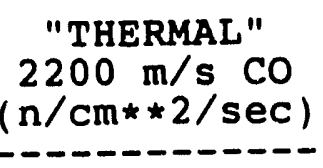

1. $09 \mathrm{E}+14$

1. $21 \mathrm{E}+14$

1. $55 \mathrm{E}+14$

1. $88 \mathrm{E}+14$

$2.18 \mathrm{E}+14$

$2.40 E+14$

$2.52 \mathrm{E}+14$

$2.65 E+14$

$2.75 E+14$

2. $90 \mathrm{E}+14$

$3.05 \mathrm{E}+14$

3. $04 \mathrm{E}+14$

$2.89 E+14$

$2.82 E+14$

$2.77 E+14$

$2.68 E+14$

$2.52 E+14$

$2.34 \mathrm{E}+14$

$2.13 E+14$

$1.78 E+14$

1. $51 \mathrm{E}+14$

1. $24 \mathrm{E}+14$

9. $56 \mathrm{E}+13$

$8.40 \mathrm{E}+13$

$5.44 \mathrm{E}+13$

$2.82 \mathrm{E}+13$

1. $30 \mathrm{E}+13$
MONITOR ID: BR-483

DATE: $03 / 15 / 93$ to $04 / 22 / 93$

"FAST"

$>1 \mathrm{MeV}$ NI

$(\mathrm{n} / \mathrm{cm} * 2 / \mathrm{sec})$

8. $30 \mathrm{E}+13$

$9.04 \mathrm{E}+13$

$1.19 E+14$

$1.41 \mathrm{E}+14$

$1.58 \mathrm{E}+14$

$1.71 \mathrm{E}+14$

$1.77 \mathrm{E}+14$

$1.81 \mathrm{E}+14$

$1.87 \mathrm{E}+14$

$1.82 \mathrm{E}+14$

$1.89 \mathrm{E}+14$

$1.91 \mathrm{E}+14$

$1.86 \mathrm{E}+14$

$1.85 \mathrm{E}+14$

$1.83 \mathrm{E}+14$

$1.84 \mathrm{E}+14$

$1.68 \mathrm{E}+14$

$1.66 \mathrm{E}+14$

$1.55 E+14$

1. $40 \mathrm{E}+14$

$1.21 \mathrm{E}+14$

$1.02 \mathrm{E}+14$

$7.19 \mathrm{E}+13$

$3.86 \mathrm{E}+13$

$1.72 \mathrm{E}+13$

$7.00 \mathrm{E}+12$

$3.61 \mathrm{E}+12$
CENTER LINE FLU. RATE

AVG. MAX. FLU. RATE

MAX . FLU. RATE/MW (AVG.)

K FACIOR

RESONANCE CORRECTION

C/L. BURNOUT CORRECTION

BACKGROUND (COUNTS/SEC.)

CHECK SOURCE (COUNTS/SEC.) $05 / 19 / 93$ OE: 33

\section{$3.04 E+14$ \\ 3. $00 \mathrm{E}+14$ \\ 1. $04 \mathrm{E}+13$ \\ 296.29 \\ 0.76 \\ 1.001 \\ 1.80}

1770 .
$1.91 \mathrm{E}+14$

$1.89 E+14$

$6.57 \mathrm{E}+12$

3706.35

NA

1.947

1.80

1770 . 
CYCLE: $100-\mathbf{A}$
EFPD: $\quad 26 \div$

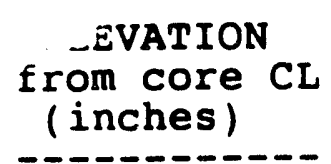

20.50

19.50

17.50

15.50

13.50

11.50

9.50

7.50

5.50

3.50

1.50

$-0.50$

$-2.50$

$-4 \cdot 50$

$-6.50$

$-8.50$

$-10.50$

$-12.50$

$-14.50$

$-16.50$

$-18.50$

$-20.50$

$-22.50$

$-24.50$

$-26.50$

$-28.50$

$-30.48$
POSITION: $W-3$

AVERAGE MW: 28.8

"THERMAL"

$2200 \mathrm{~m} / \mathrm{s} \mathrm{CO}$

$(\mathrm{n} / \mathrm{cm} * \star 2 / \mathrm{sec})$

$1.10 E+14$

$1.23 \mathrm{E}+14$

$1.64 \mathrm{E}+14$

$1.97 \mathrm{E}+14$

$2.33 \mathrm{E}+14$

$2.50 \mathrm{E}+14$

$2.62 \mathrm{E}+14$

$2.74 \mathrm{E}+14$

$2.81 \mathrm{E}+14$

$2.97 \mathrm{E}+14$

$3.08 E+14$

$3.02 \mathrm{E}+14$

$2.86 \mathrm{E}+14$

$2.91 E+14$

$2.81 E+14$

$2.75 \mathrm{E}+14$

$2.62 E+14$

$2.48 \mathrm{E}+14$

$2.28 \mathrm{E}+14$

$1.93 \mathrm{E}+14$

$1.64 \mathrm{E}+14$

$1.34 \mathrm{E}+14$

$1.02 \mathrm{E}+14$

7. $29 \mathrm{E}+13$

4. $26 E+13$

$2.31 \mathrm{E}+13$

$1.23 E+13$
MONITOR ID: BR-484

DATE : $03 / 15 / 93$ to $04 / 22 / 93$

"FAST"

$>1 \mathrm{MeV}$ NI

$(\mathrm{n} / \mathrm{cm} \star \star 2 / \mathrm{sec})$

3. $80 \mathrm{E}+13$

$4.23 E+13$

$5.68 \mathrm{E}+13$

$6.42 \mathrm{E}+13$

$7.18 \mathrm{E}+13$

8. $07 \mathrm{E}+13$

8. $44 \mathrm{E}+13$

$8.73 \mathrm{E}+13$

8. $68 \mathrm{E}+13$

9. $30 E+13$

9. $12 \mathrm{E}+13$

9. $10 \mathrm{E}+13$

8.97E+13

$9.47 \mathrm{E}+13$

$9.21 E+13$

$8.56 \mathrm{E}+13$

8. $43 \mathrm{E}+13$

$7.77 \mathrm{E}+13$

$7.65 \mathrm{E}+13$

$6.70 \mathrm{E}+13$

$5.69 \mathrm{E}+13$

$4.77 \mathrm{E}+13$

$3.44 \mathrm{E}+13$

$2.12 \mathrm{E}+13$

1. $10 \mathrm{E}+13$

$4.97 \mathrm{E}+12$

$2.70 \mathrm{E}+12$
CENTER LINE FLU. RATE

AVG. MAX. FLU. RATE

MAX. FLU. RATE/MW (AVG.)

K FACTOR

RESONANCE CORRECTION

$C / L$. BURNOUT CORRECTION

BACKGROUND (COUNTS/SEC.)

CHECK SOURCE (COUNTS/SEC .)
$3.02 \mathrm{E}+14$

$3.02 \mathrm{E}+14$

$1.05 E+13$

291.83

0.81

1.001

1.80

1757.
9. $10 \mathrm{E}+13$

9. $22 \mathrm{E}+13$

3. $20 \mathrm{E}+12$

3610.89

NA

1.940

1.80

1757. 
CYCLE: 100-A

EFPD: 20.6

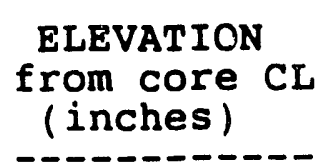

20.50

19.50

17.50

15.50

13.50

11.50

9.50

7.50

5.50

3.50

1.50

$-0.50$

$-2.50$

$-4.50$

$-6.50$

$-8.50$

$-10.50$

$-12.50$

$-14.50$

$-16.50$

$-18.50$

$-20.50$

$-22.50$

$-24.50$

$-26.50$

$-28.50$

$-30.48$
POSITION: W-4

AVERAGE MW: 28.8

"THERMAL"

$2200 \mathrm{~m} / \mathrm{s}$ CO

$(\mathrm{n} / \mathrm{cm} * \star 2 / \mathrm{sec})$

$1.18 \mathrm{E}+14$

1. $32 E+14$

$1.70 \mathrm{E}+14$

2. $10 \mathrm{E}+14$

$2.36 \mathrm{E}+14$

$2.55 \mathrm{E}+14$

$2.69 \mathrm{E}+14$

$2.83 E+14$

$2.91 \mathrm{E}+14$

$3.04 \mathrm{E}+14$

$3.15 \mathrm{E}+14$

3. $12 \mathrm{E}+14$

3. $00 \mathrm{E}+14$

$2.97 \mathrm{E}+14$

$2.92 E+14$

$2.83 E+14$

$2.69 \mathrm{E}+14$

$2.52 \mathrm{E}+14$

$2.24 \mathrm{E}+14$

$1.99 \mathrm{E}+14$

1. $63 \mathrm{E}+14$

1. $30 \mathrm{E}+14$

1. $00 \mathrm{E}+14$

7. $38 \mathrm{E}+13$

4. $36 \mathrm{E}+13$

$2.34 E+13$

1. $20 E+13$
MONITOR ID: BR-485

DATE: $03 / 15 / 93$ to $04 / 22 / 93$

"FAST"

$>1 \mathrm{MeV} N \mathrm{NI}$

$(\mathrm{n} / \mathrm{cm} \star \star 2 / \mathrm{sec})$

4. $29 E+13$

$4.89 \mathrm{E}+13$

$6.36 \mathrm{E}+13$

$7.37 \mathrm{E}+13$

8.09E+13

$8.63 \mathrm{E}+13$

$9.21 \mathrm{E}+13$

$9.27 \mathrm{E}+13$

$9.05 \mathrm{E}+13$

$9.48 \mathrm{E}+13$

$9.48 \mathrm{E}+13$

$9.49 E+13$

$9.59 \mathrm{E}+13$

$9.42 \mathrm{E}+13$

9. $36 \mathrm{E}+13$

$9.28 \mathrm{E}+13$

8. $90 \mathrm{E}+13$

8. $31 \mathrm{E}+13$

8. $00 \mathrm{E}+13$

$7.33 \mathrm{E}+13$

$6.17 \mathrm{E}+13$

4. $90 \mathrm{E}+13$

$3.75 E+13$

$2.19 E+13$

1. $10 \mathrm{E}+13$

$4.98 \mathrm{E}+12$

$2.61 \mathrm{E}+12$
CENTER LINE FLU. RATE

AVG. MAX. FLU. RATE

MAX. FLU. RATE/MW (AVG.)

K FACTOR

RESONANCE CORRECTION

C/L. BURNOUT CORRECTION

BACKGROUND (COUNTS/SEC.)

CHECK SOURCE (COUNTS/SEC.)

05/20/93 08:30
3. $12 \mathrm{E}+14$

3. $10 \mathrm{E}+14$

$1.08 \mathrm{E}+13$

290.57

0.76

1.001

1.67

1757 .
$9.49 \mathrm{E}+13$

$9.52 \mathrm{E}+13$

$3.31 \mathrm{E}+12$

3725.24

NA

1.972

1.67

1757. 
$\begin{array}{ll}\text { CYCLE: } & 100-\mathrm{A} \\ \text { EFPD: } & 20.6\end{array}$

ELEVATION
from core CL
(inches)

25.23

23.25

21.25

19.25

17.25

15.25

13.25

11.25

9.25

7.25

5.25

3.25

1.25

$-0.75$

$-2.75$

$-4.75$

$-6.75$

$-8.75$

$-10.75$

$-12.75$

$-14.75$

$-16.75$

$-18.75$

$-20.75$

$-22.75$

$-23.75$
POSITION: $\mathrm{H}-2$

AVEEAGE MW: 32.7

"THERMAL"

$2200 \mathrm{~m} / \mathrm{s} \mathrm{CO}$

$(\mathrm{n} / \mathrm{cm} * * 2 / \mathrm{sec})$

$7.06 \mathrm{E}+13$

$7.62 \mathrm{E}+13$

8. $99 E+13$

1. $13 \mathrm{E}+14$

1. $38 \mathrm{E}+14$

1. $64 \mathrm{E}+14$

$1.93 \mathrm{E}+14$

$2.12 \mathrm{E}+14$

2. $23 E+14$

$2.35 E+14$

$2.33 E+14$

$2.41 E+14$

$2.38 E+14$

$2.34 \mathrm{E}+14$

$2.36 E+14$

$2.35 \mathrm{E}+14$

$2.33 E+14$

2. $30 \mathrm{E}+14$

$2.20 \mathrm{E}+14$

$2.06 \mathrm{E}+14$

$1.78 \mathrm{E}+14$

1. $58 \mathrm{E}+14$

1. $35 E+14$

1. $11 \mathrm{E}+14$

$9.48 \mathrm{E}+13$

$9.23 E+13$
MONITOR ID: H-200

DATE: $03 / 15 / 93$ to $04 / 22 / 93$

"FAST"

$>1 \mathrm{MeV}$ NI

$(\mathrm{n} / \mathrm{cm} \star \star 2 / \mathrm{sec})$

$2.61 \mathrm{E}+13$

$4.15 E+13$

$6.96 \mathrm{E}+13$

8. $93 E+13$

$1.01 \mathrm{E}+14$

1. $15 \mathrm{E}+14$

1. $35 E+14$

1. $45 E+14$

1. $50 \mathrm{E}+14$

$1.54 \mathrm{E}+14$

$1.58 \mathrm{E}+14$

1. $55 \mathrm{E}+14$

$1.58 \mathrm{E}+14$

$1.60 \mathrm{E}+14$

$1.62 \mathrm{E}+14$

$1.61 \mathrm{E}+14$

$1.59 \mathrm{E}+14$

$1.56 \mathrm{E}+14$

1. $51 \mathrm{E}+14$

1. $34 \mathrm{E}+14$

1. $34 \mathrm{E}+14$

$1.14 \mathrm{E}+14$

$1.02 \mathrm{E}+14$

8. $14 \mathrm{E}+13$

$5.29 \mathrm{E}+13$

$4.44 E+13$
CENTER LINE FLU. RATE

AVG. MAX. FLU. RATE

MAX . FLU. RATE/MW (AVG.)

K FACTOR

RESONANCE CORRECTION

$C / L$. BURNOUT CORRECTION

BACKGROUND (COUNTS/SEC.)

CHECK SOURCE (COUNTS/SEC.)
$2.34 \mathrm{E}+14$

$2.38 \mathrm{E}+14$

7. $27 \mathrm{E}+12$

282.48

0.75

1.001

1.59

1770 .
1. $60 \mathrm{E}+14$

1. $61 \mathrm{E}+14$

$4.92 \mathrm{E}+12$

3605.17

NA

1.739

1.59

1770 . 
CYCLE: $100-\mathrm{A}$
EFPD: 20.6

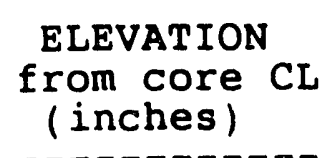

25.23

23.25

21.25

19.25

17.25

15.25

13.25

11.25

9.25

7.25

5.25

3.25

1.25

$-0.75$

$-2.75$

$-4.75$

$-6.75$

$-8.75$

$-10.75$

$-12.75$

$-14.75$

$-16.75$

$-18.75$

$-20.75$

$-22.75$

$-23.75$
POSITION: $\mathrm{H}-10$

AVERAGE MW: 32.7

"THERMAL"
$2200 \mathrm{~m} / \mathrm{s} \mathrm{cO}$
$\mathrm{n} / \mathrm{cm} * 2 / \mathrm{sec})$

$7.64 E+13$

8. $19 \mathrm{E}+13$

9. $64 \mathrm{E}+13$

1. $19 \mathrm{E}+14$

1. $46 \mathrm{E}+14$

$1.72 \mathrm{E}+14$

$2.04 E+14$

$2.19 \mathrm{E}+14$

$2.30 \mathrm{E}+14$

$2.40 \mathrm{E}+14$

$2.39 \mathrm{E}+14$

$2.43 E+14$

$2.41 \mathrm{E}+14$

$2.37 E+14$

$2.41 \mathrm{E}+14$

$2.37 E+14$

$2.39 E+14$

$2.34 E+14$

$2.31 E+14$

$2.20 E+14$

$1.98 \mathrm{E}+14$

$1.69 \mathrm{E}+14$

$1.45 \mathrm{E}+14$

$1.19 \mathrm{E}+14$

$9.99 \mathrm{E}+13$

$1.01 \mathrm{E}+14$
MONITOR ID: H-201

DATE: $03 / 15 / 93$ to $04 / 22 / 93$

"FAST"

$>1 \mathrm{MeV}$ NI

$(\mathrm{n} / \mathrm{cm} \star \star 2 / \mathrm{sec})$

$2.81 E+13$

$4.64 \mathrm{E}+13$

$7.88 \mathrm{E}+13$

$1.02 \mathrm{E}+14$

1. $22 \mathrm{E}+14$

1. $38 \mathrm{E}+14$

1. $54 \mathrm{E}+14$

1. $64 \mathrm{E}+14$

$1.71 \mathrm{E}+14$

$1.85 \mathrm{E}+14$

$1.88 \mathrm{E}+14$

2. $00 E+14$

1. $93 \mathrm{E}+14$

1. $94 \mathrm{E}+14$

1. $92 \mathrm{E}+14$

1. $92 \mathrm{E}+14$

1. $88 \mathrm{E}+14$

1. $70 \mathrm{E}+14$

1. $57 \mathrm{E}+14$

1. $64 \mathrm{E}+14$

1. $49 \mathrm{E}+14$

1. $32 E+14$

1. $12 \mathrm{E}+14$

$9.13 E+13$

$6.64 \mathrm{E}+13$

$5.06 \mathrm{E}+13$
CENTER LINE FLU. RATE

AVG. MAX. FLU. RATE

MAX.FLU. RATE/MW (AVG.)

K FACTOR

RESONANCE CORRECTION

$\mathrm{C} / \mathrm{L}$. BURNOUT CORRECTION

BACKGROUND (COUNTS/SEC.)

CHECK SOURCE (COUNTS/SEC.)

05/19/93 08:33
$2.37 \mathrm{E}+14$

$2.41 \mathrm{E}+14$

$7.36 \mathrm{E}+12$

290.13

0.75

1.001

1.85

1770 .
$1.94 \mathrm{E}+14$

$1.96 \mathrm{E}+14$

$5.98 \mathrm{E}+12$

3452.97

NA

1.749

1.85

1770 . 

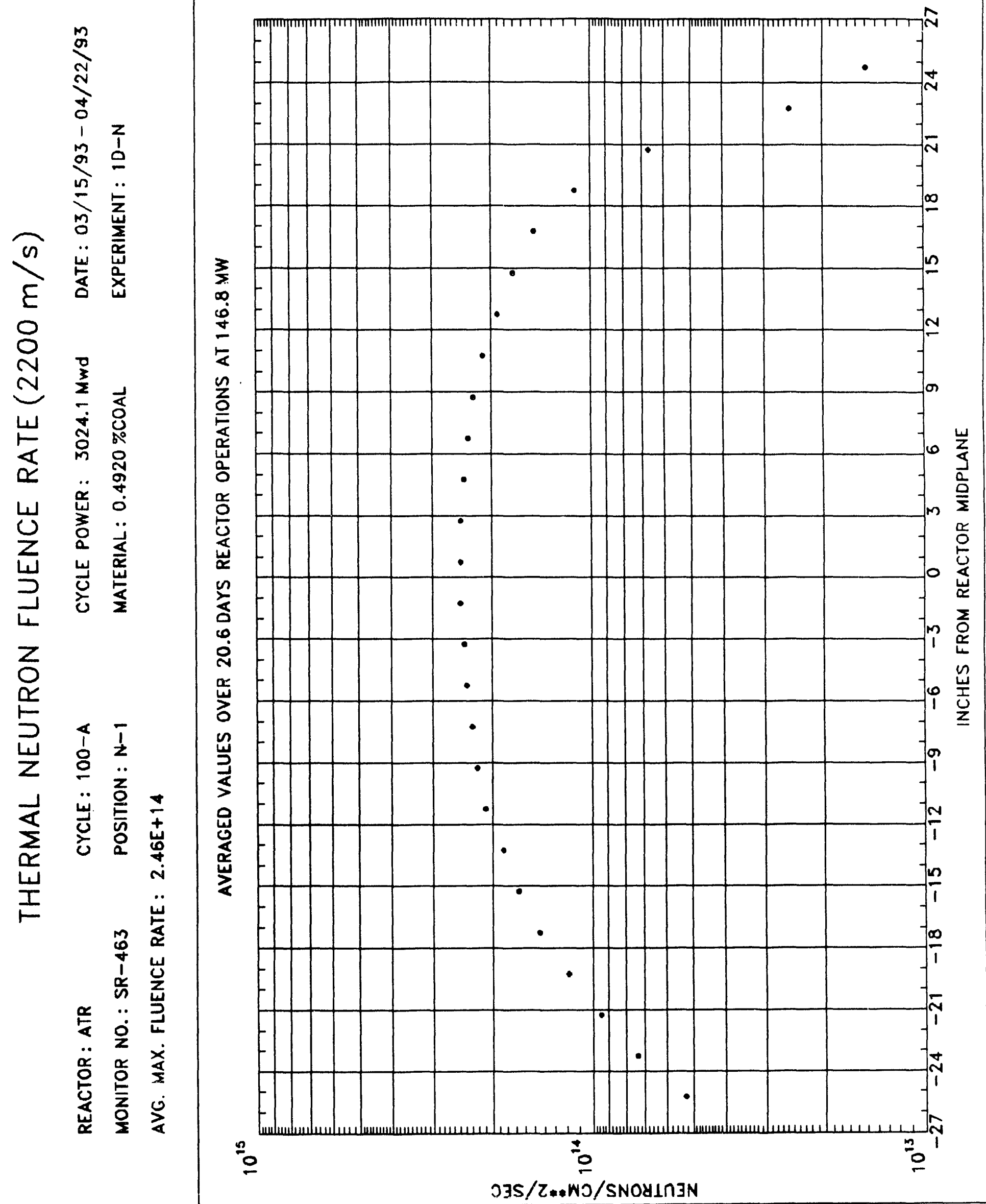

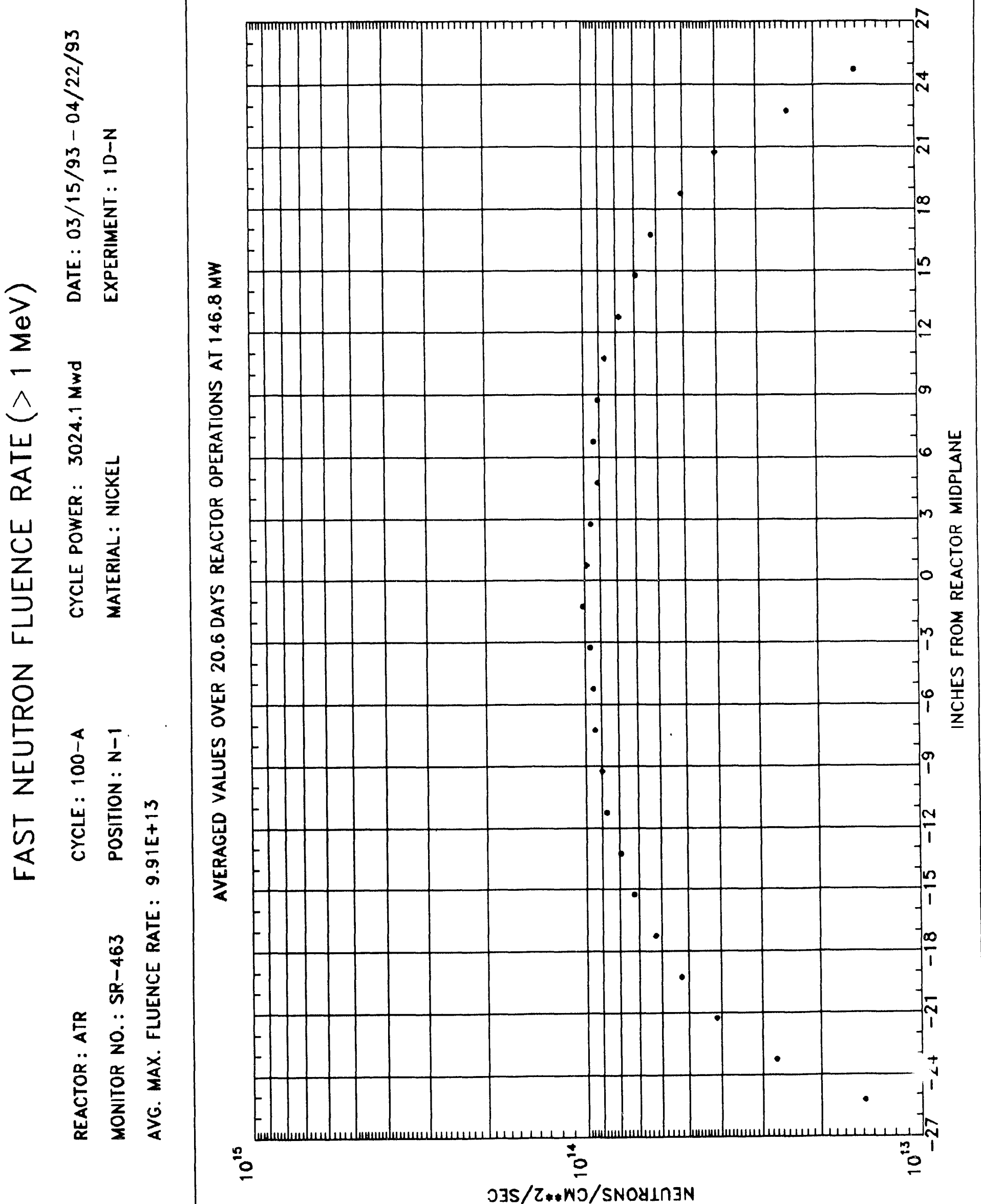


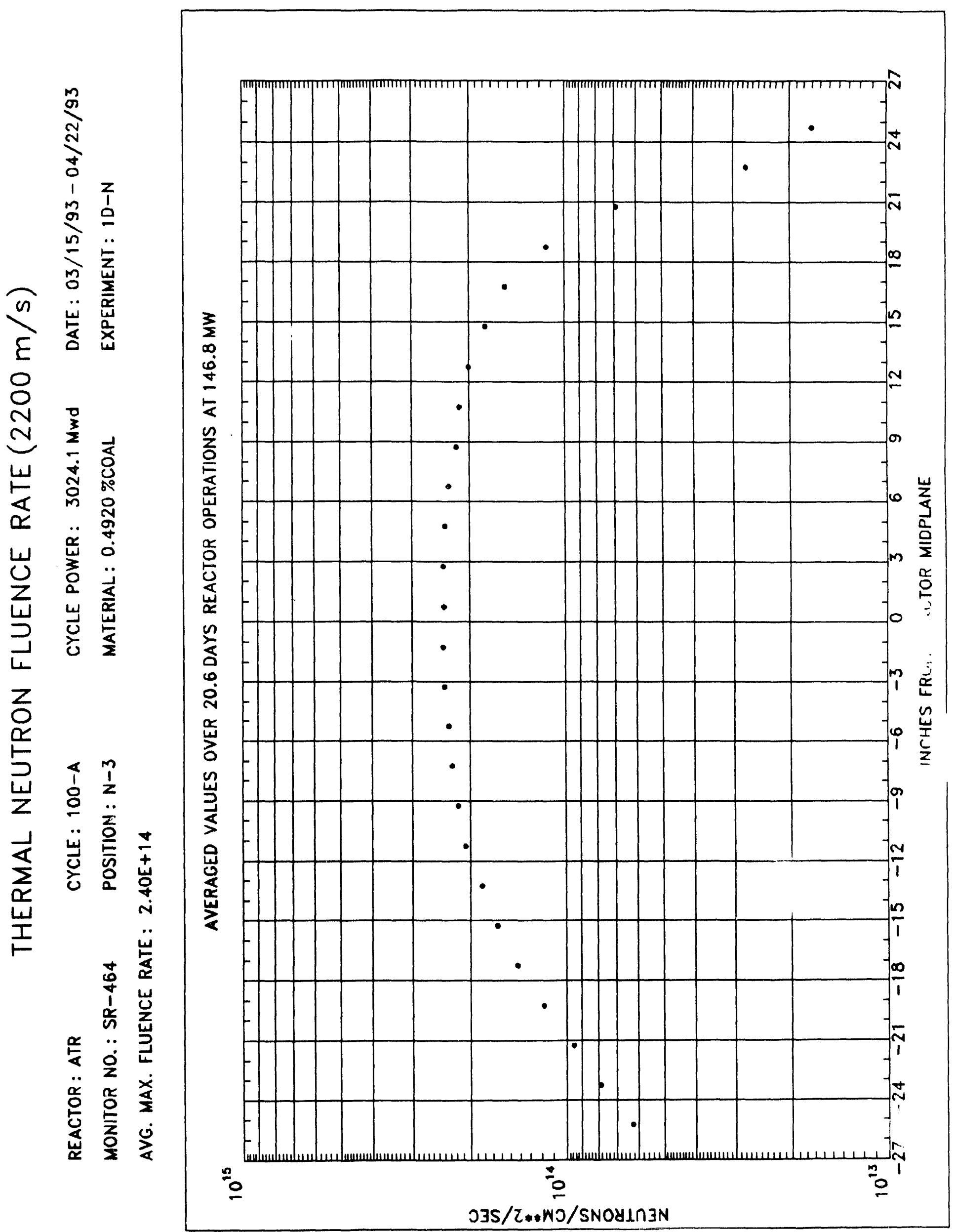




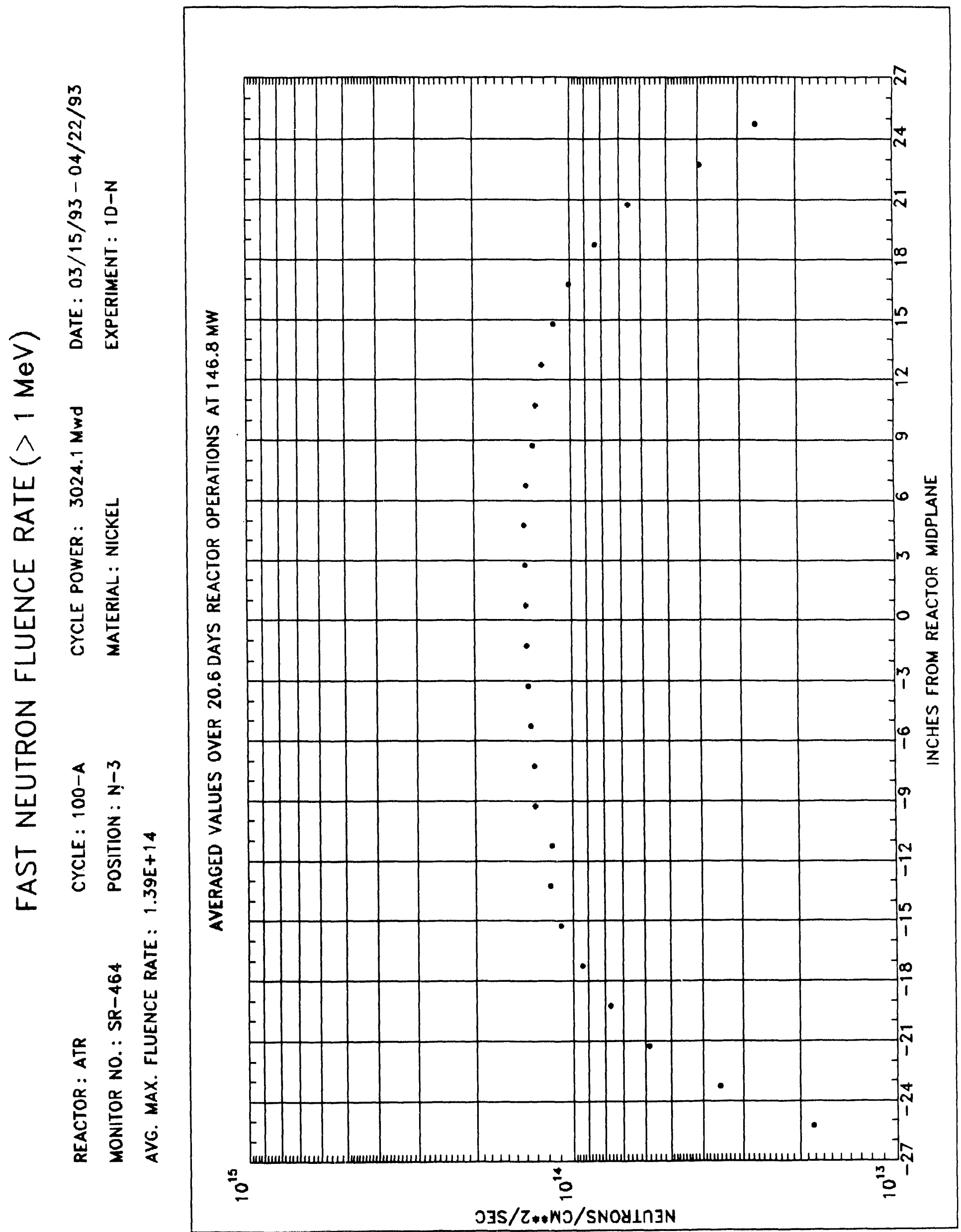




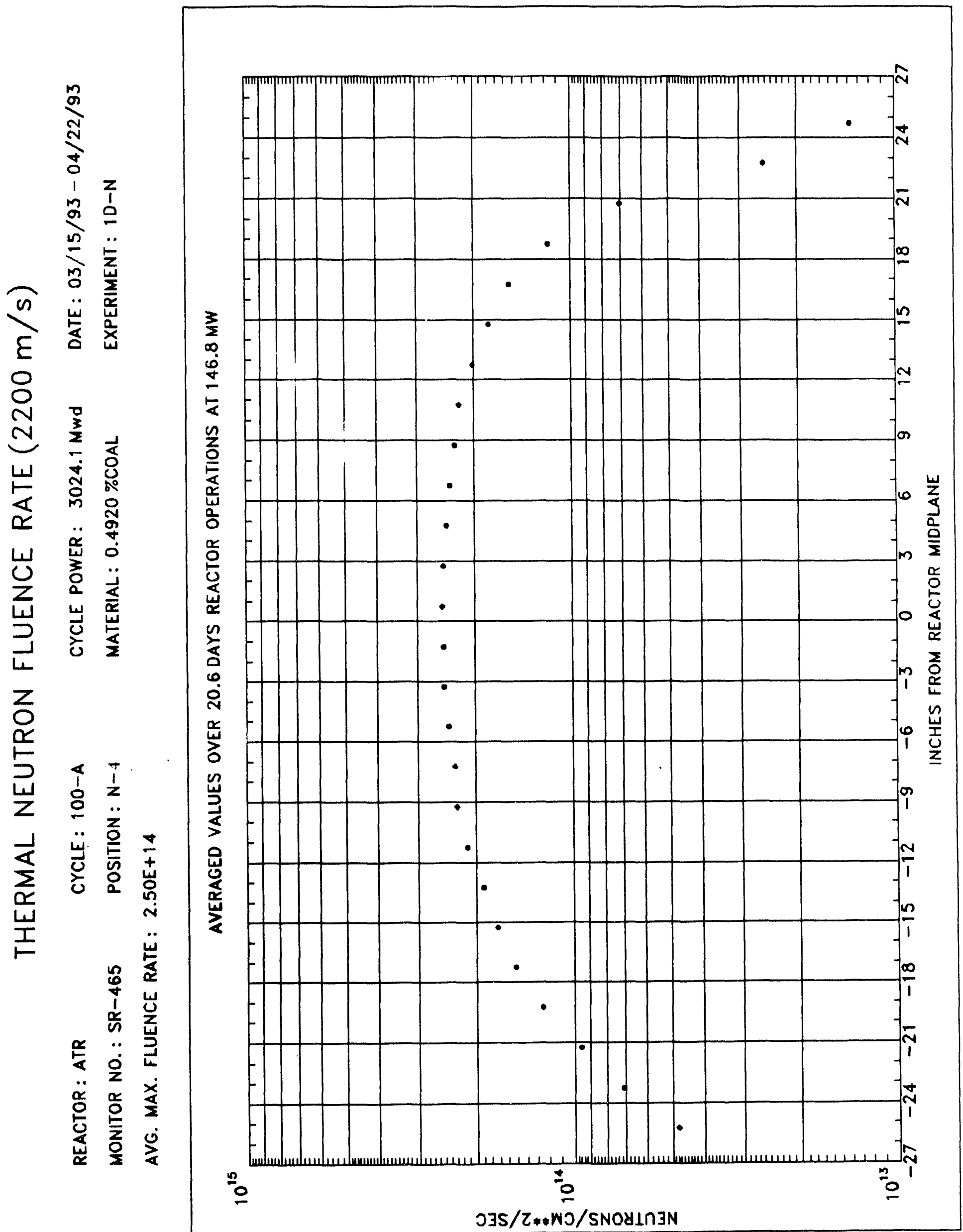



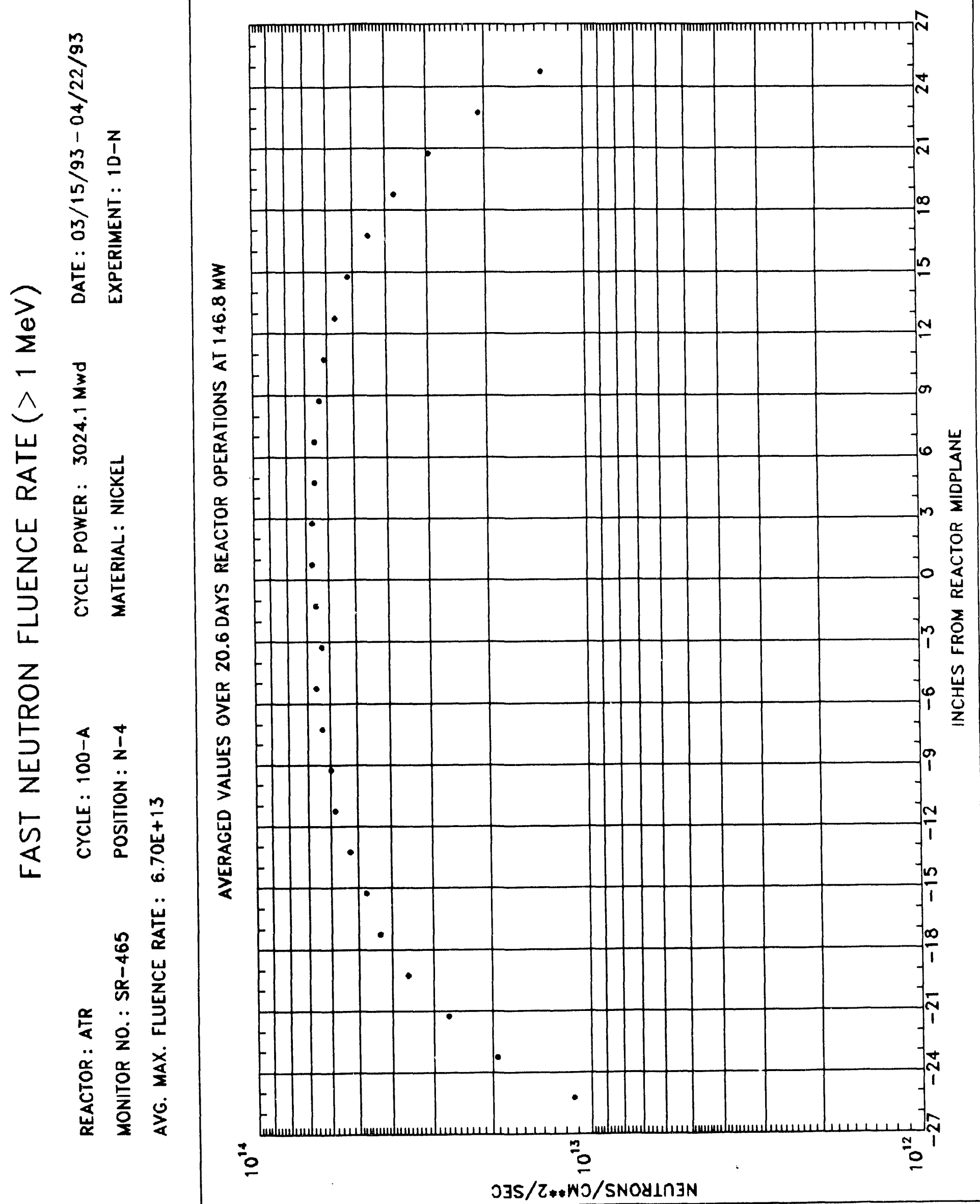


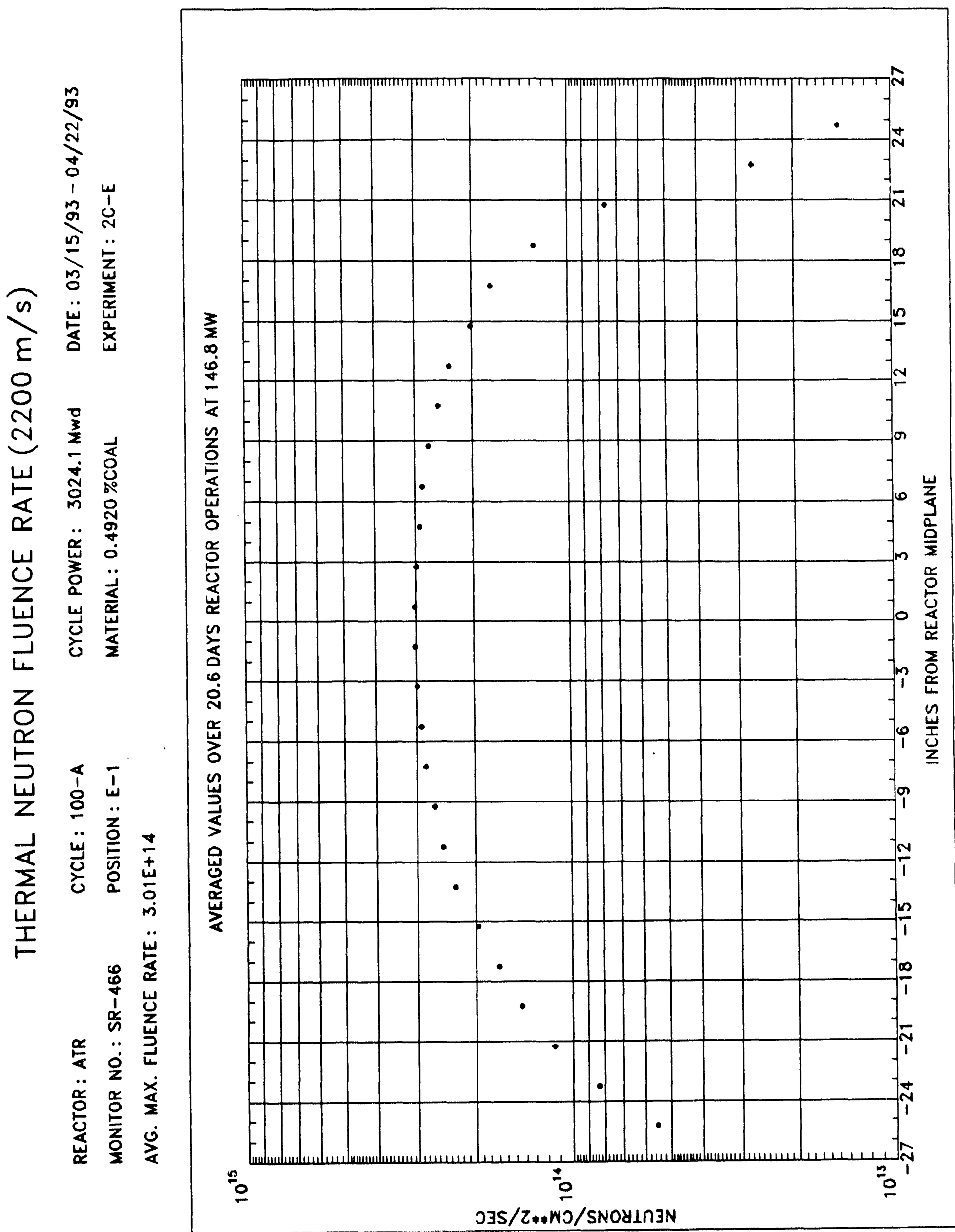




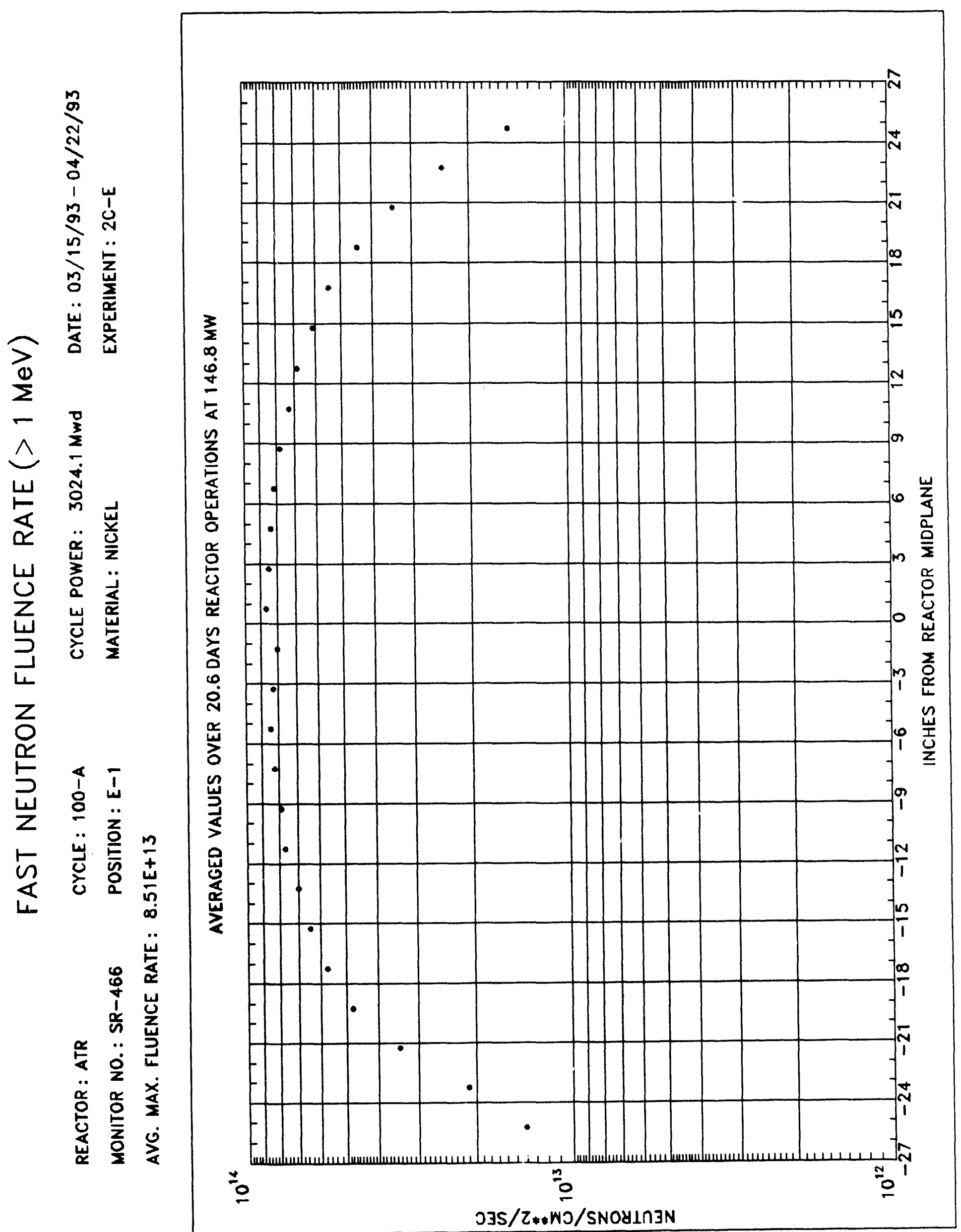




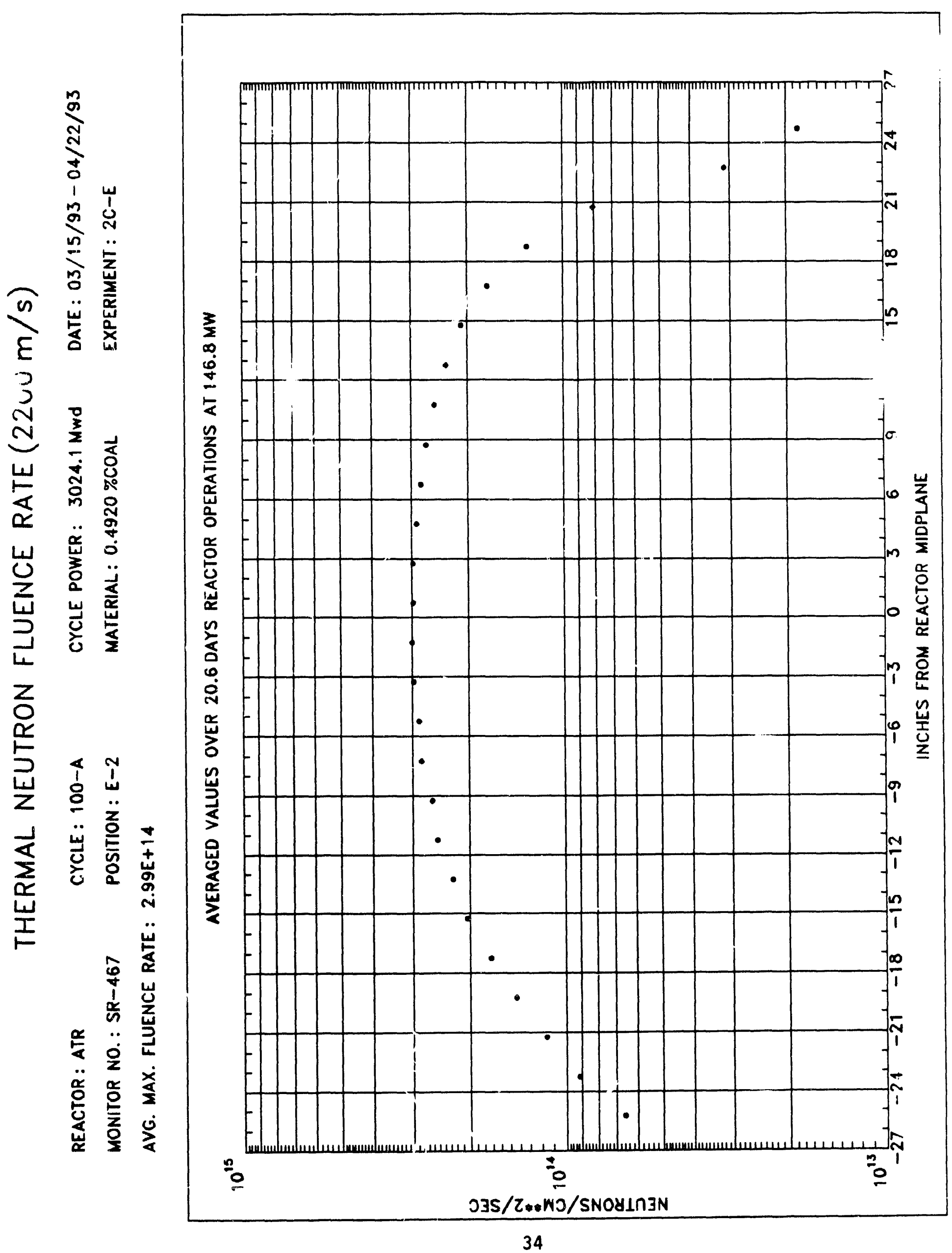




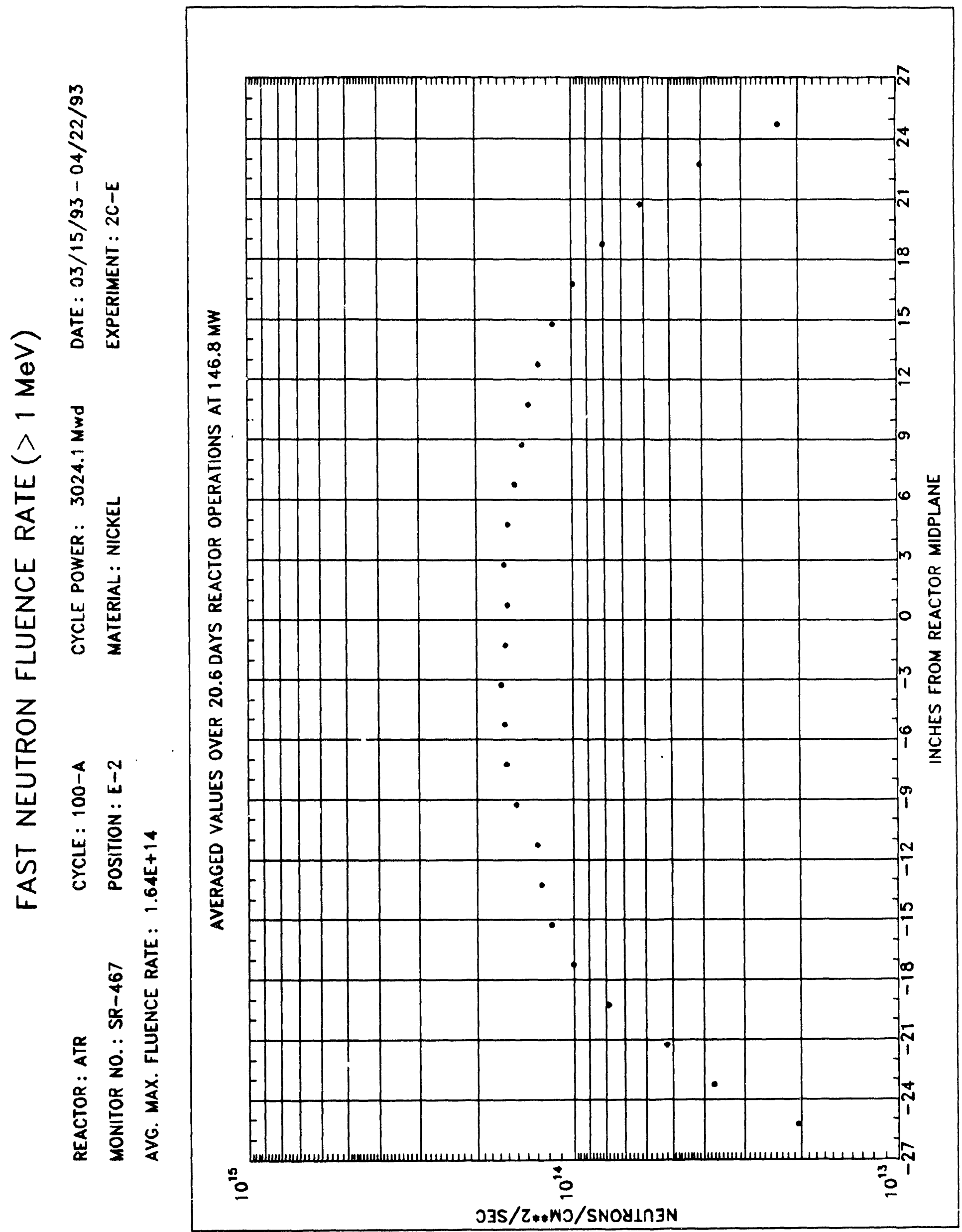



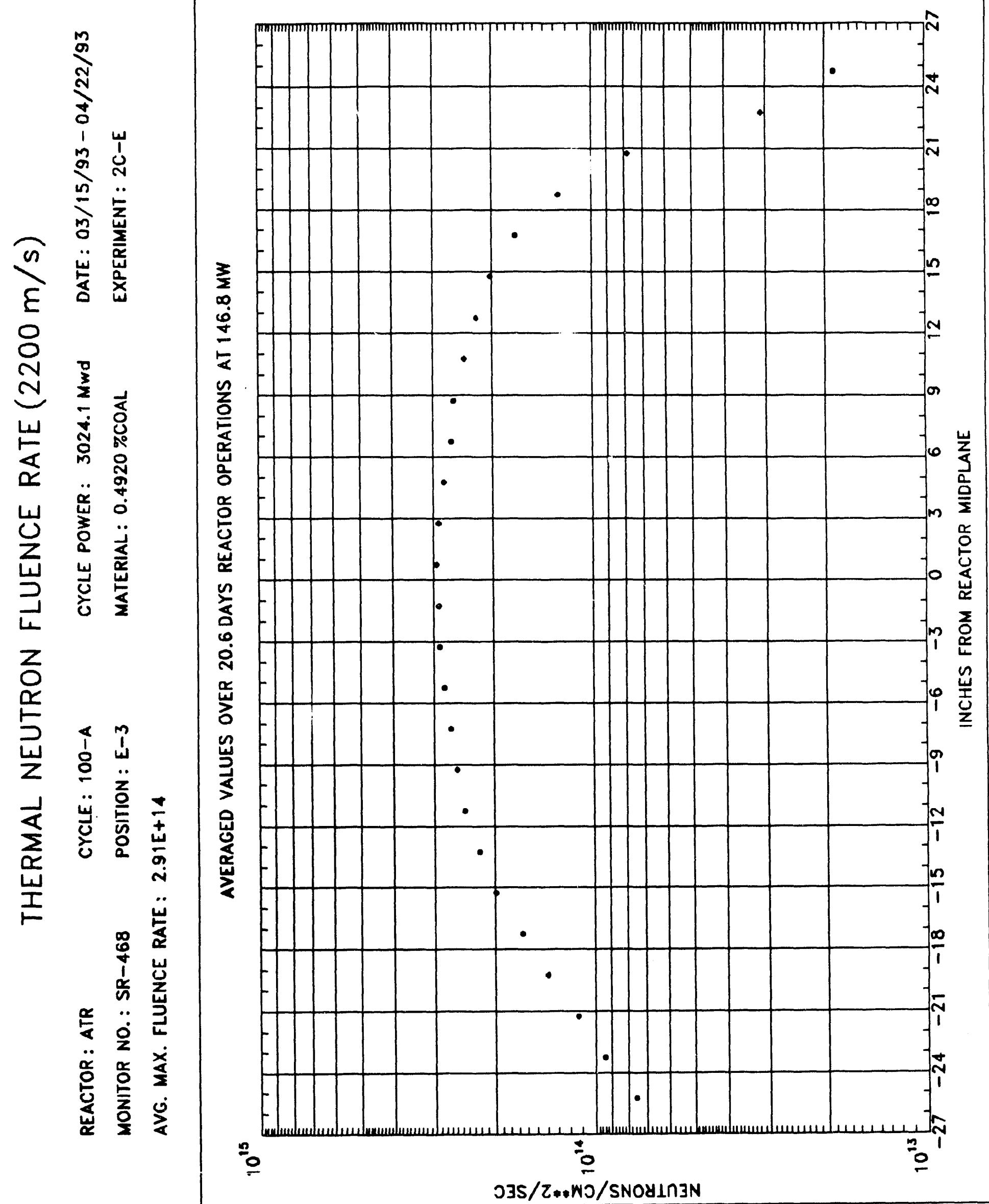

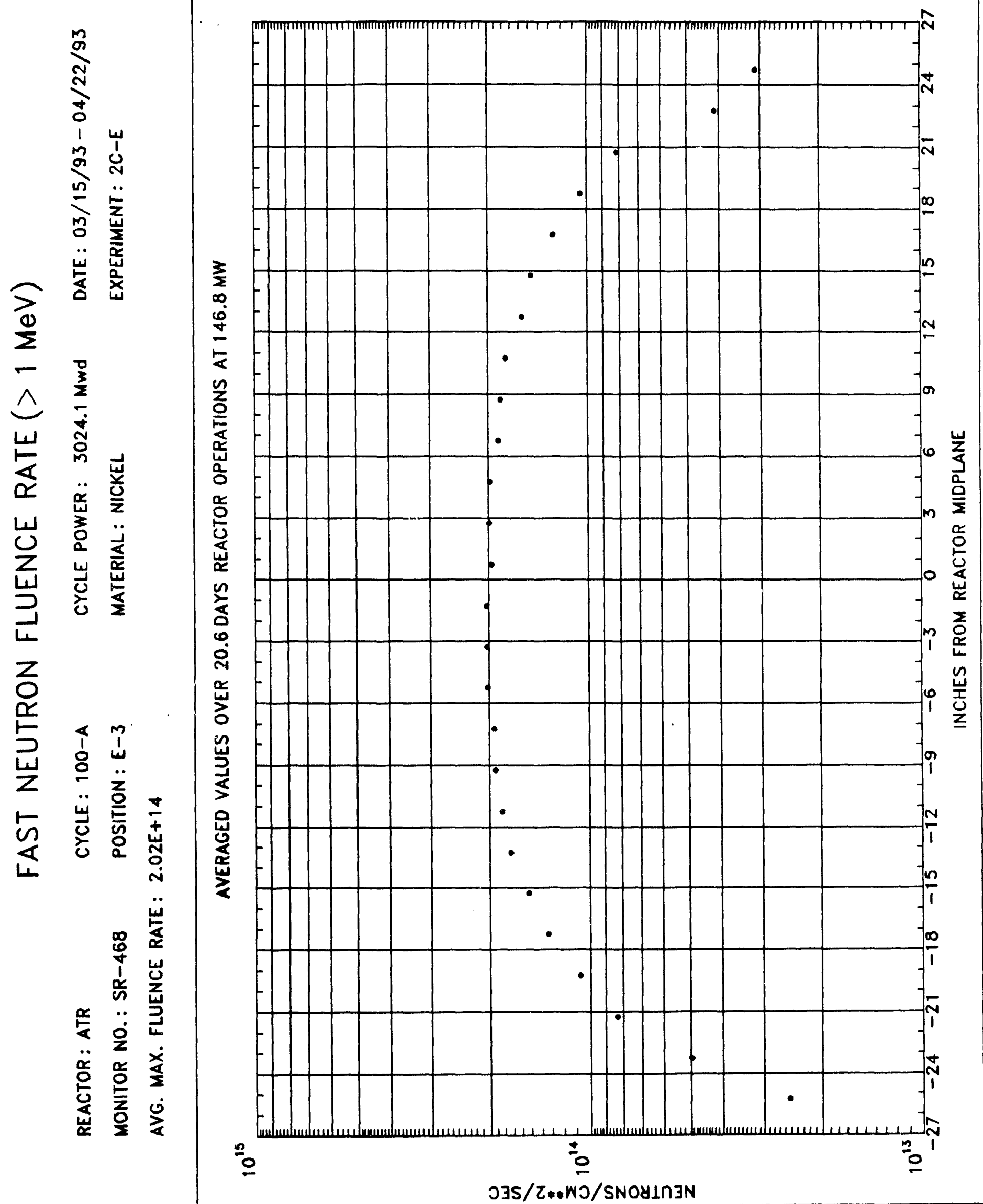

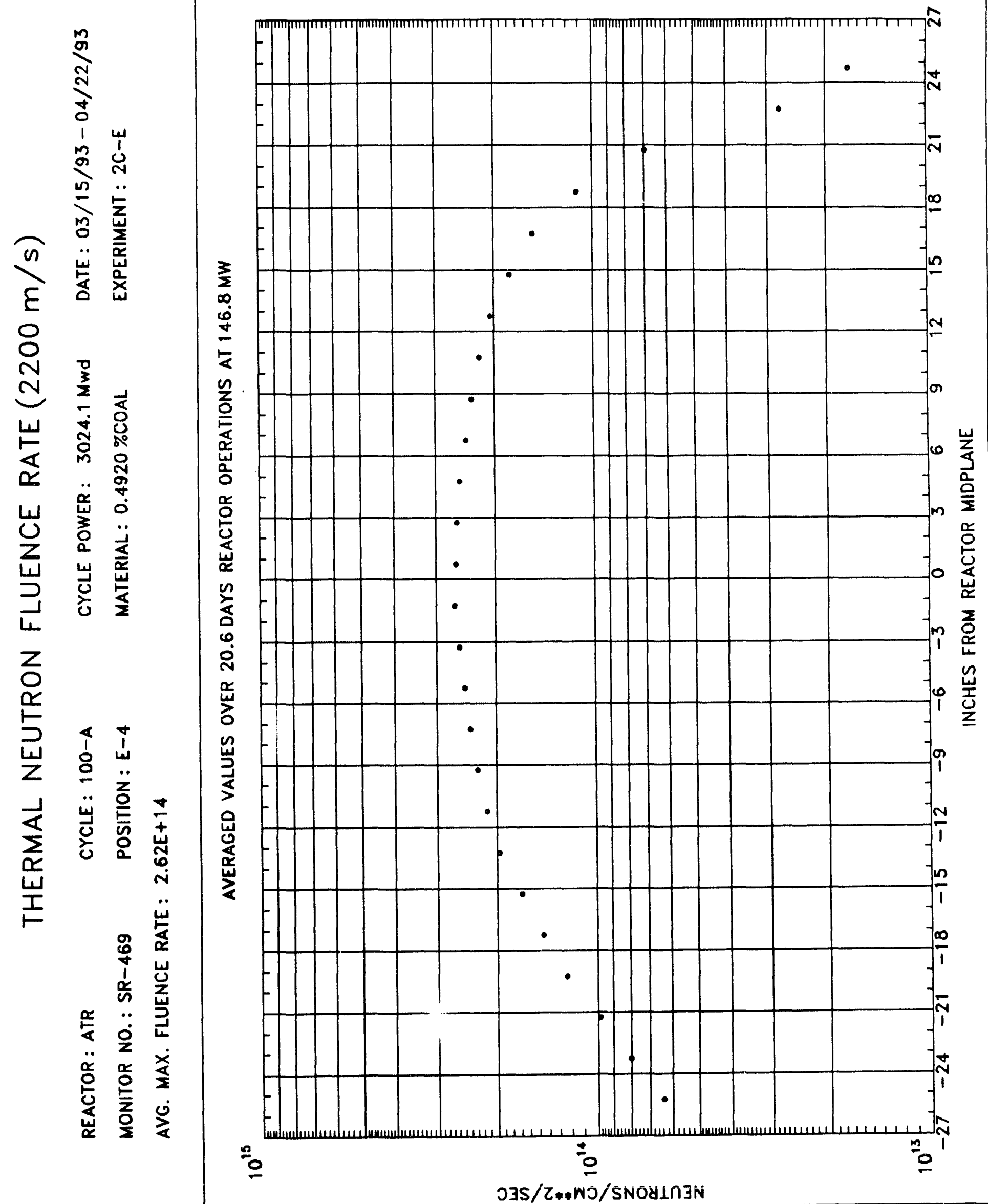

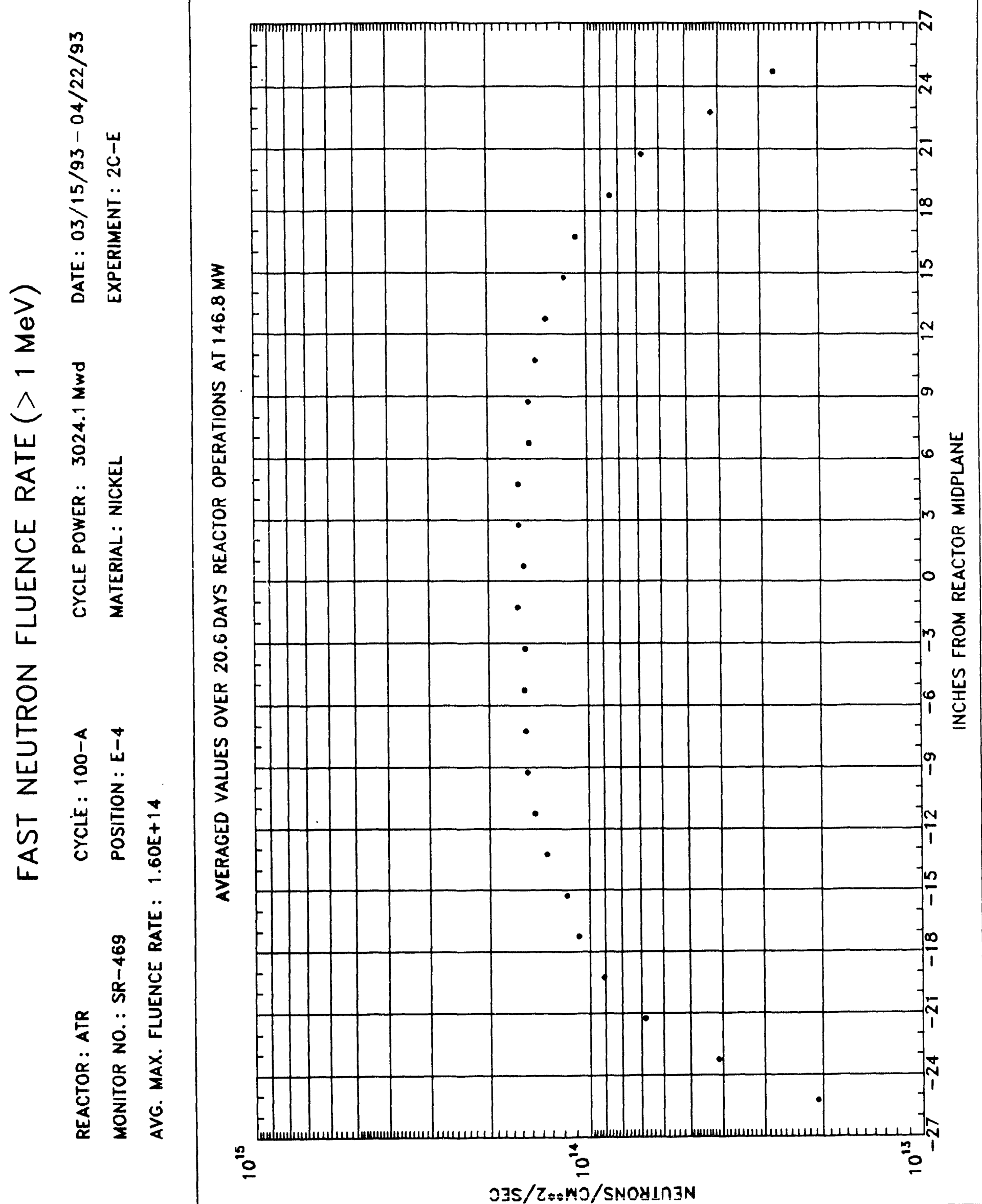

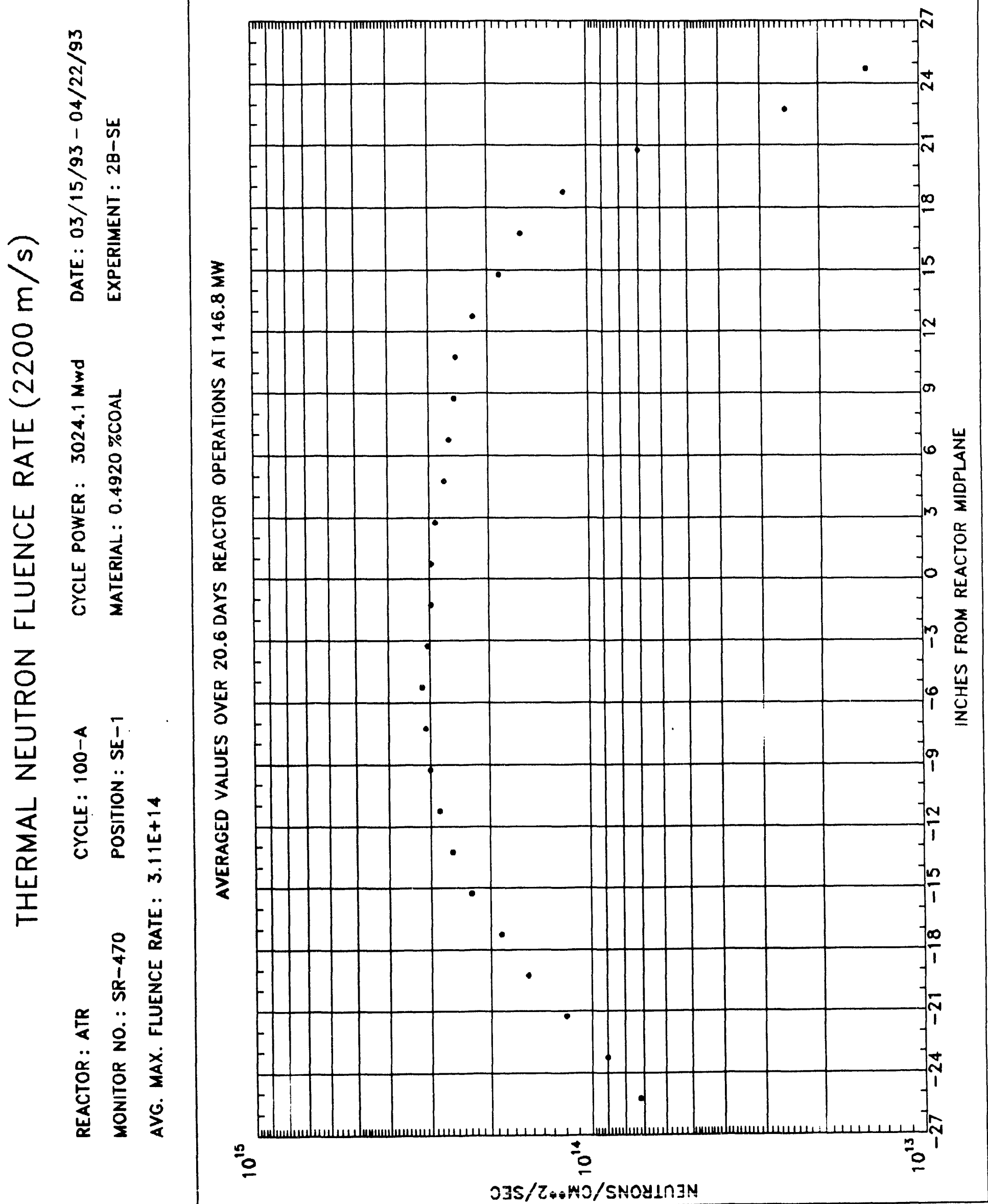


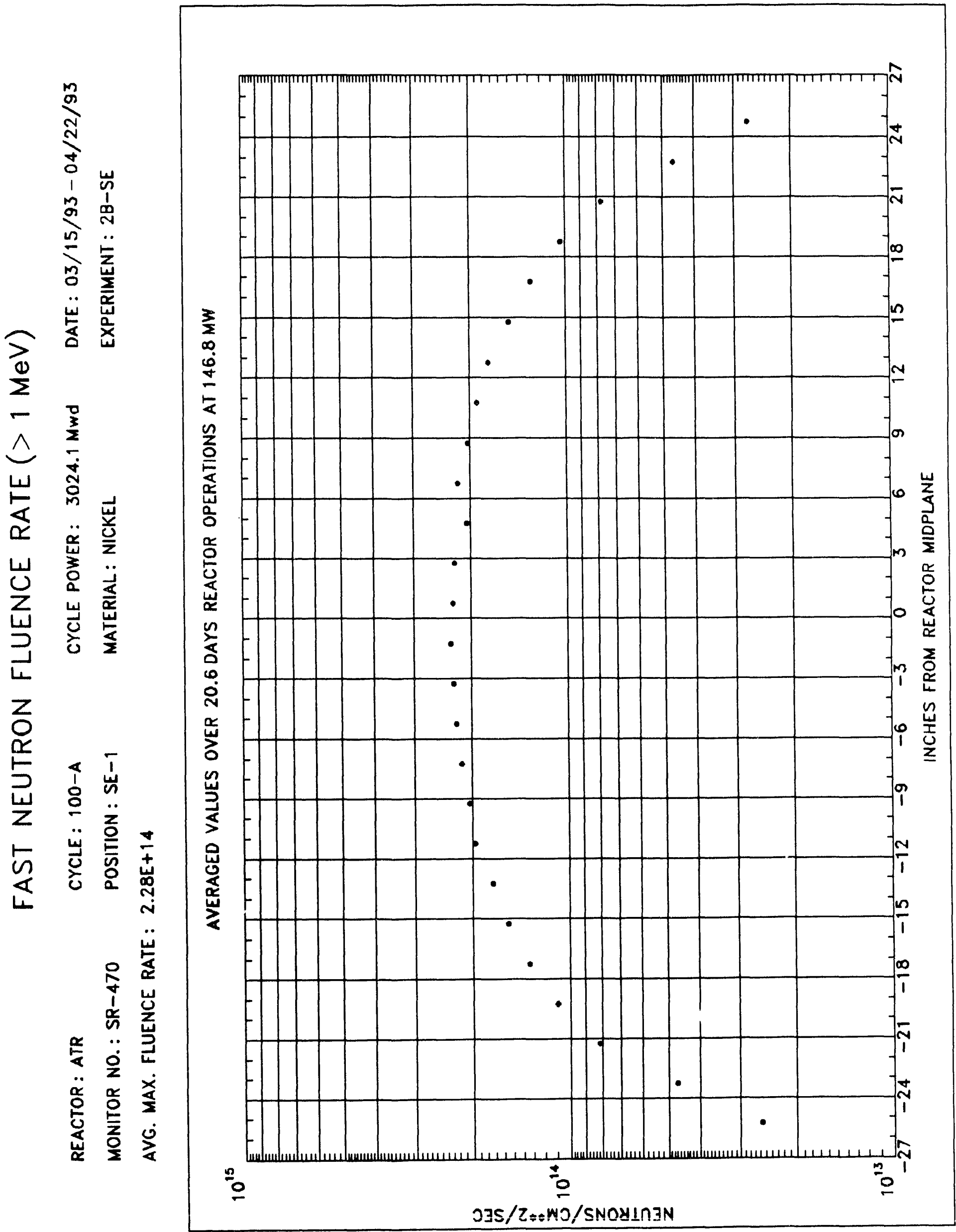



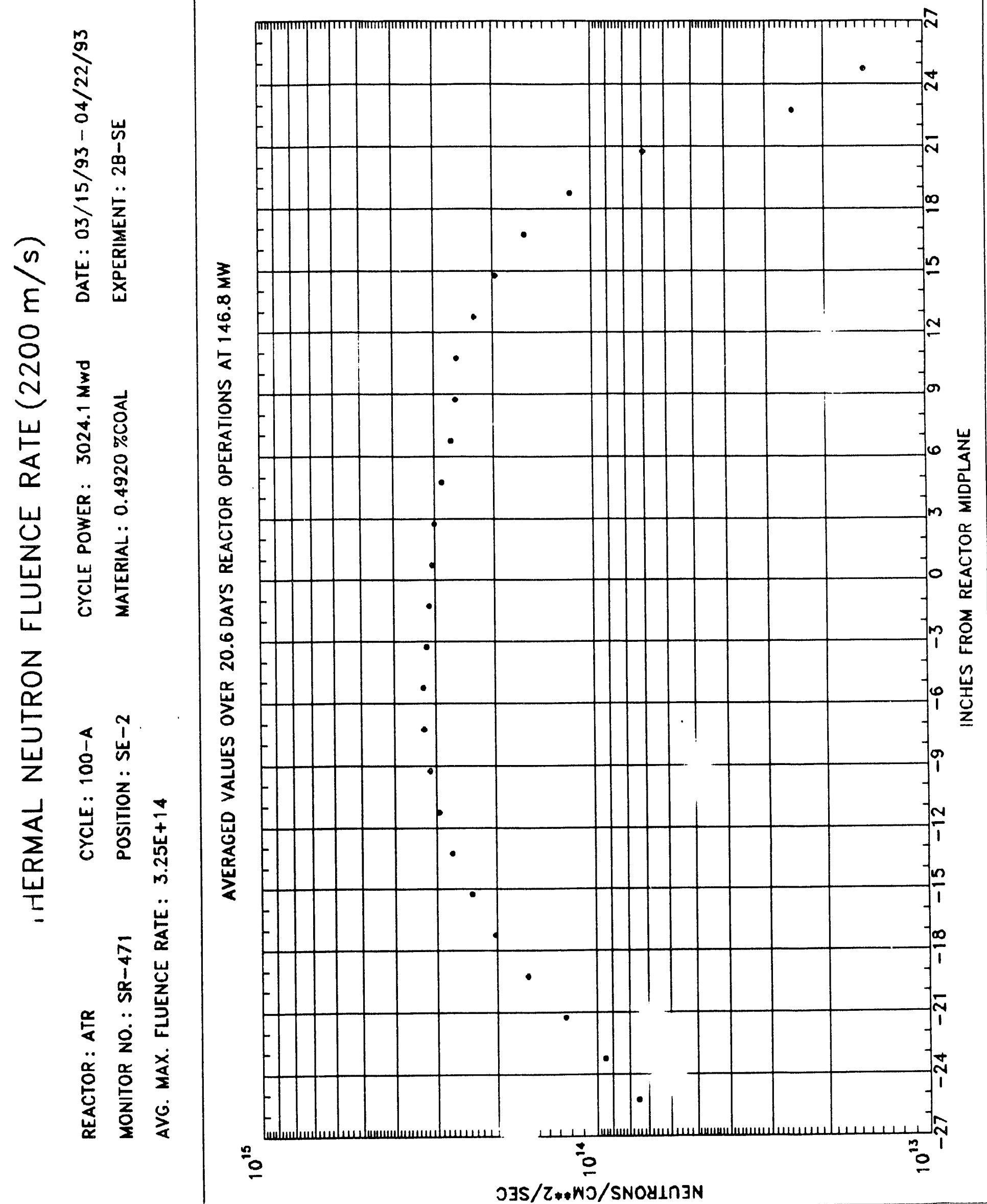


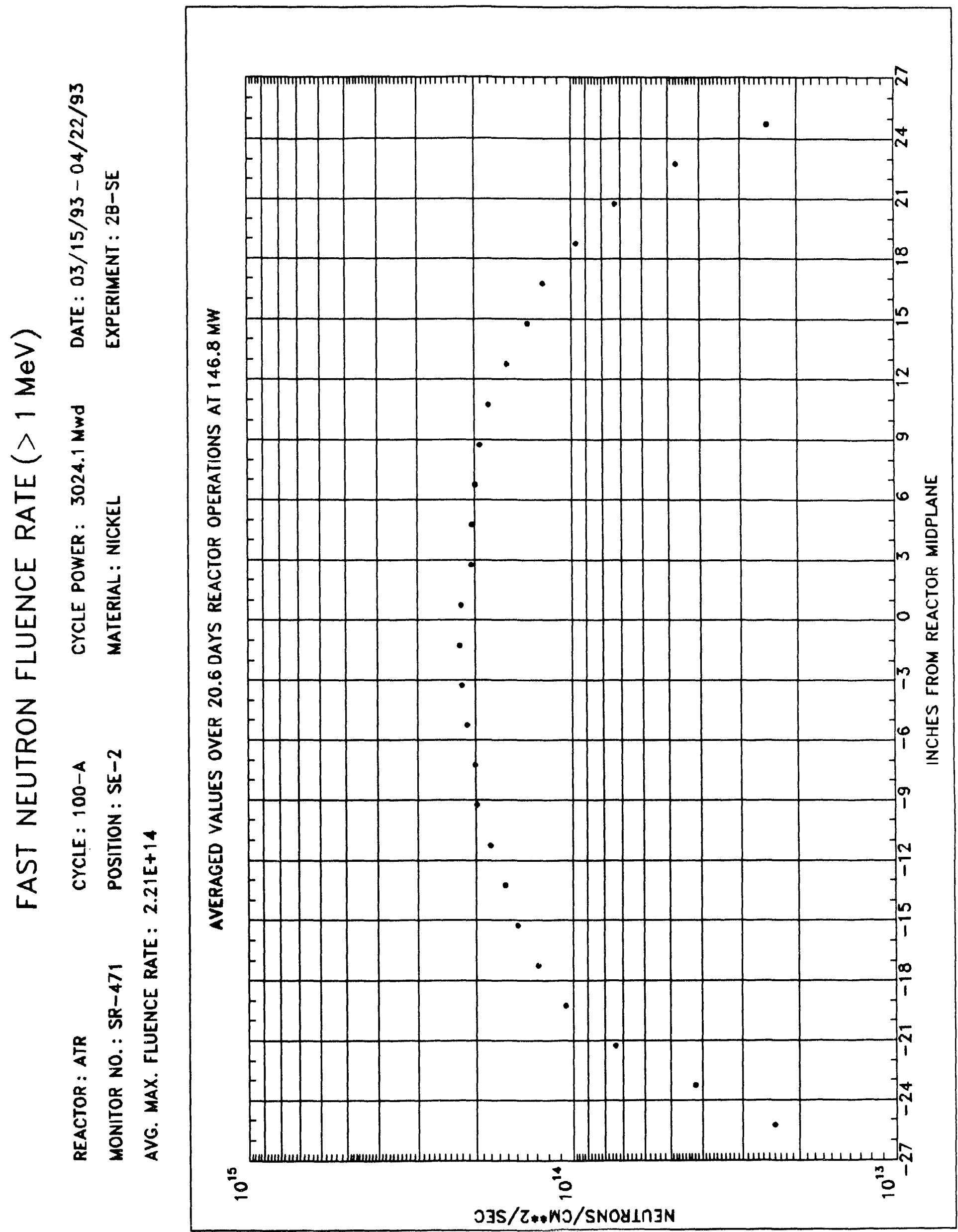



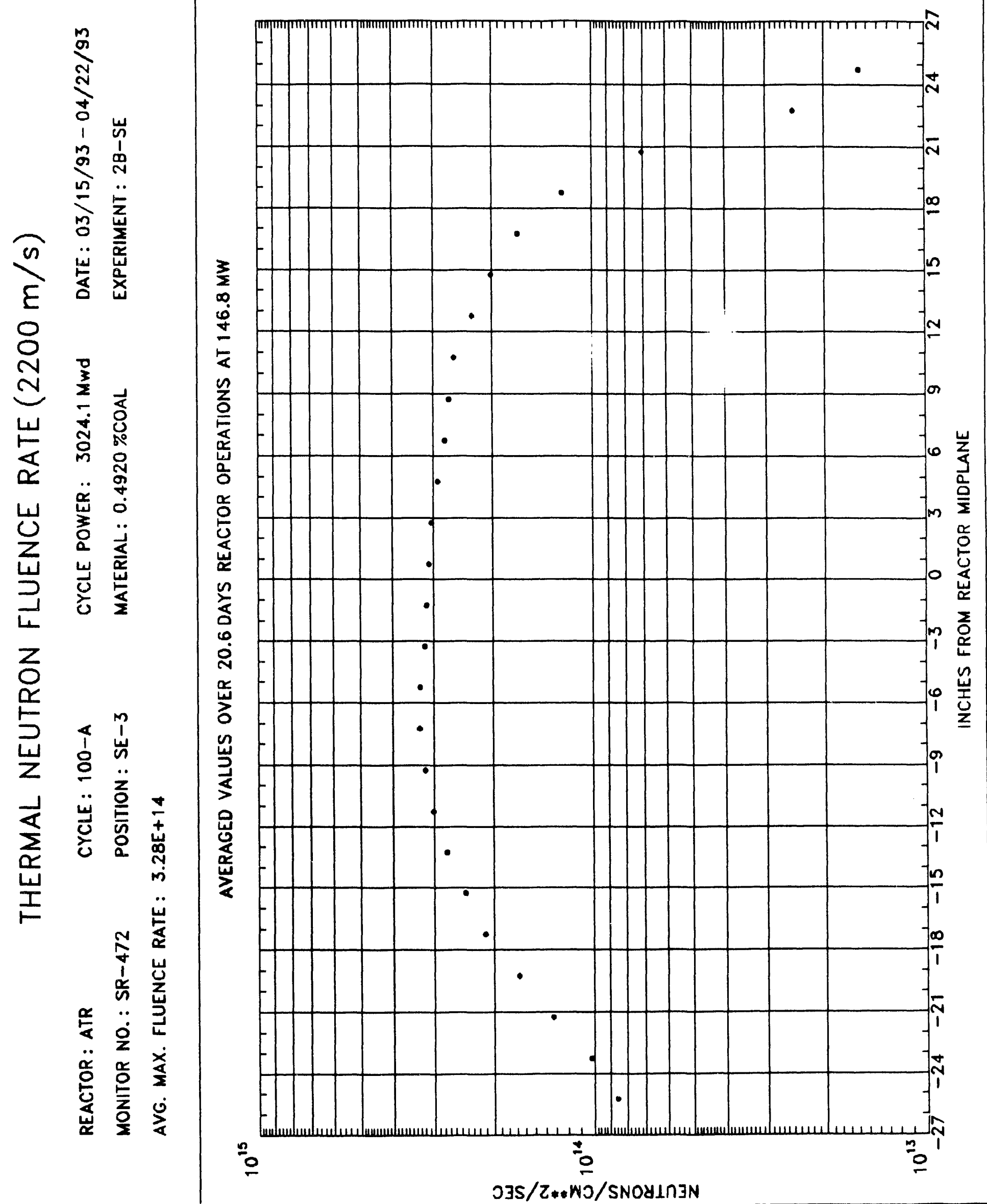


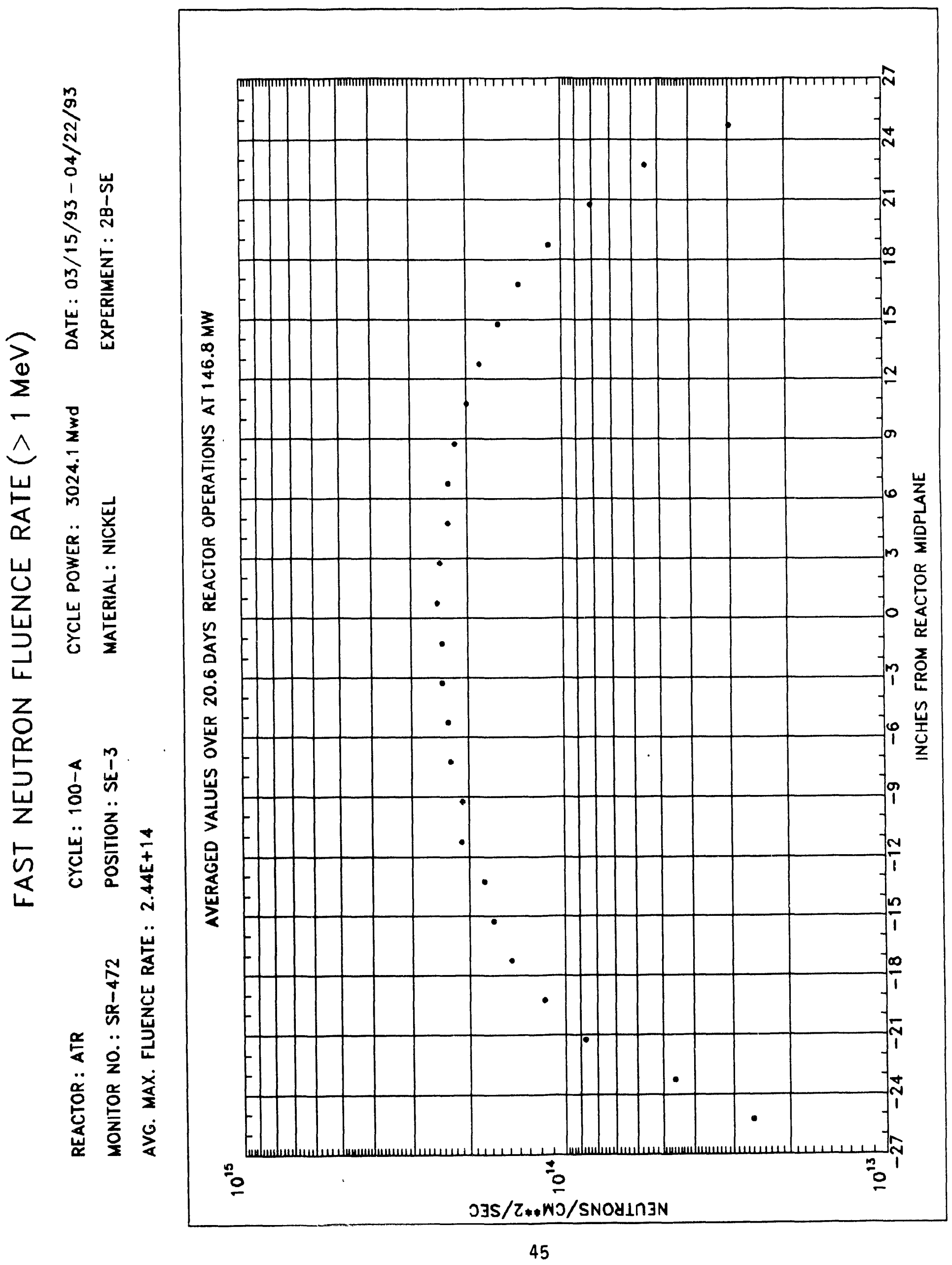



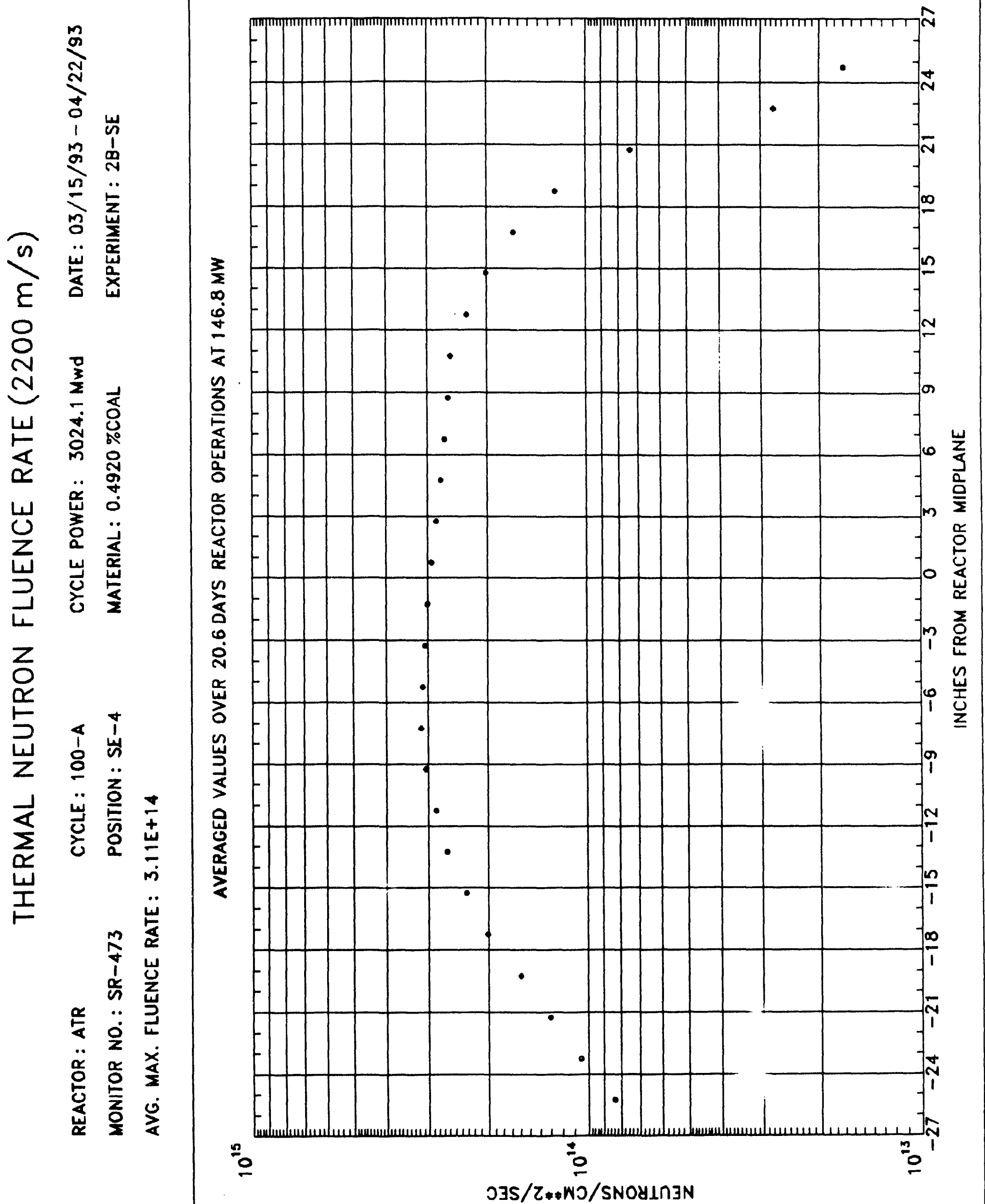

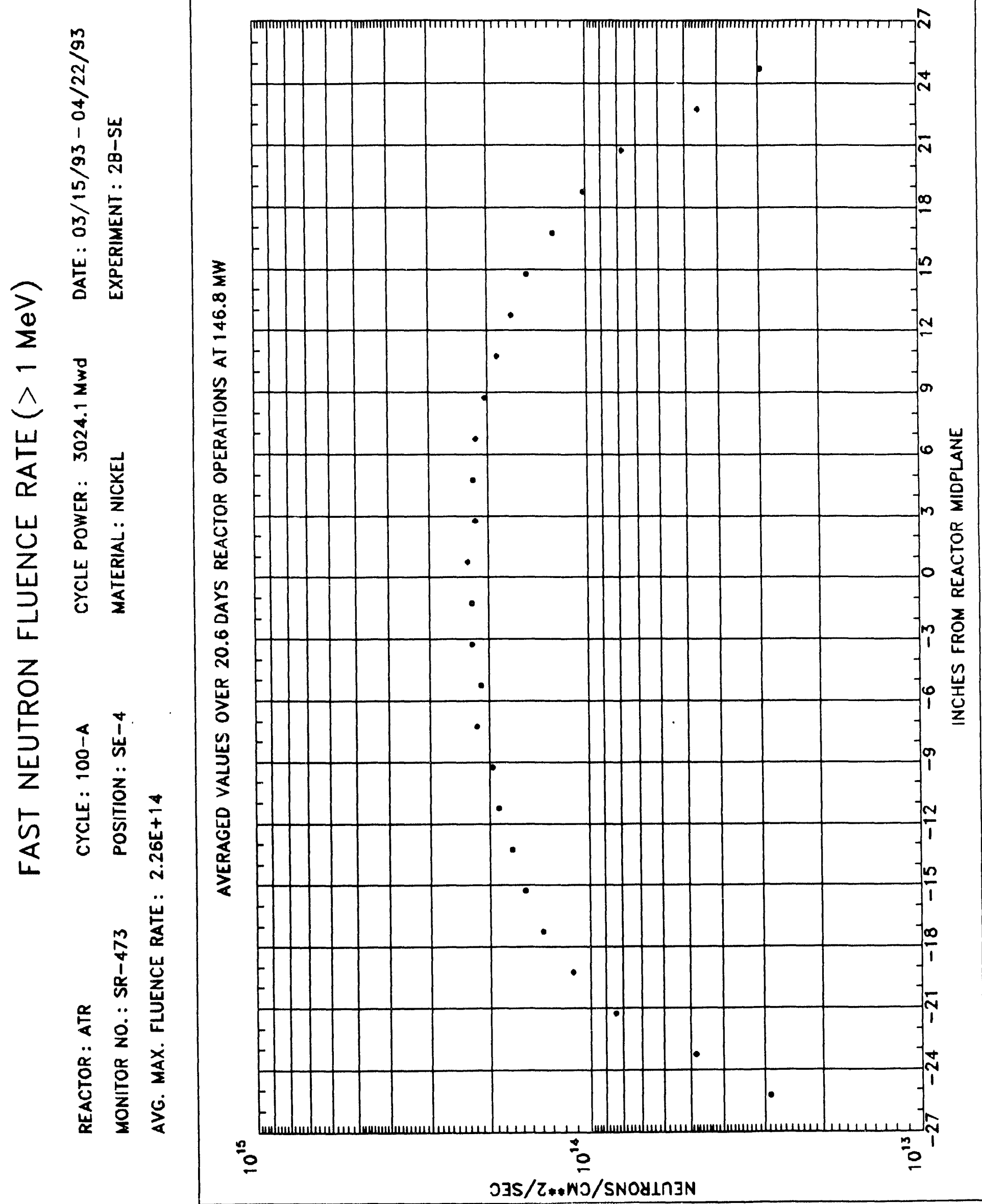


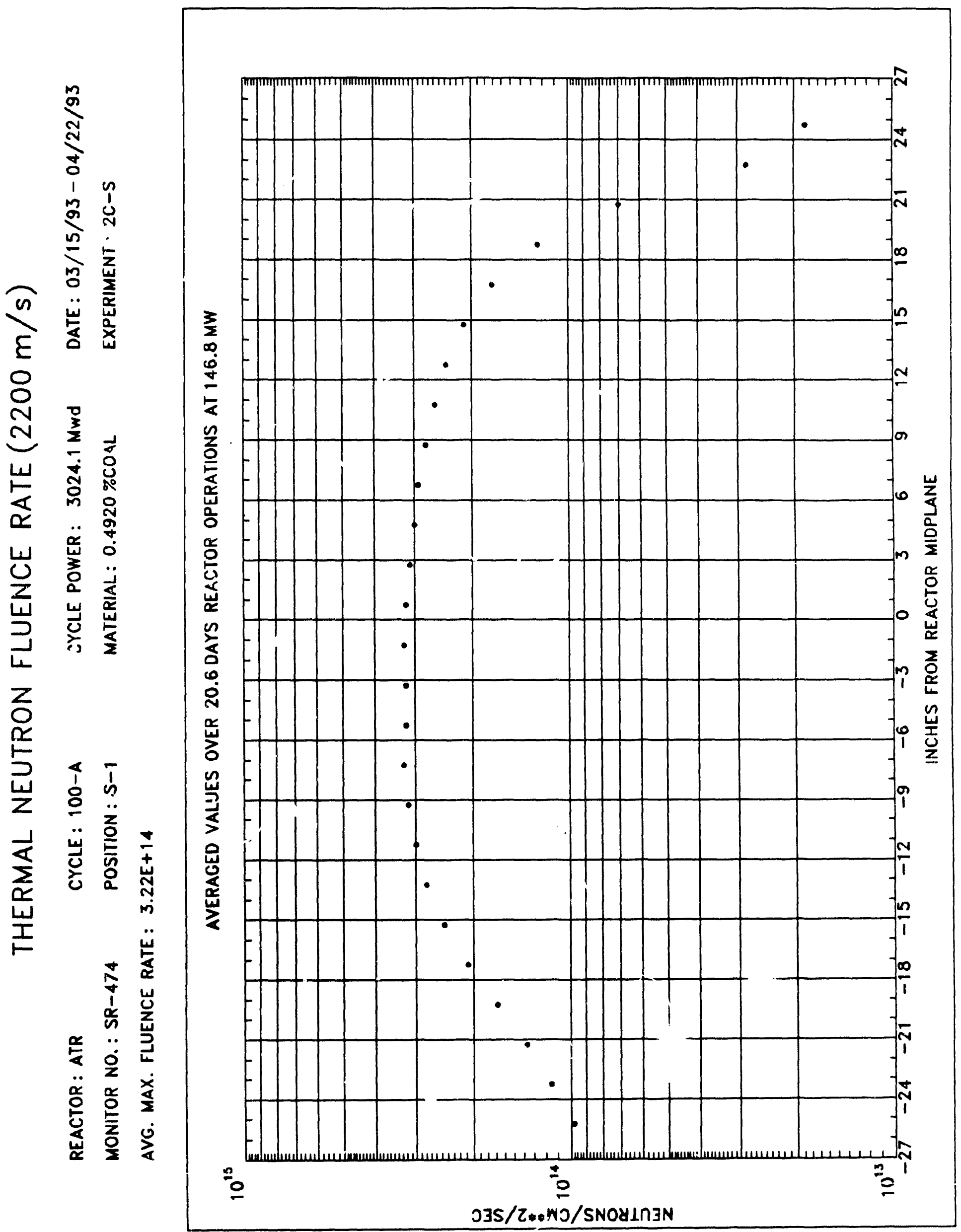



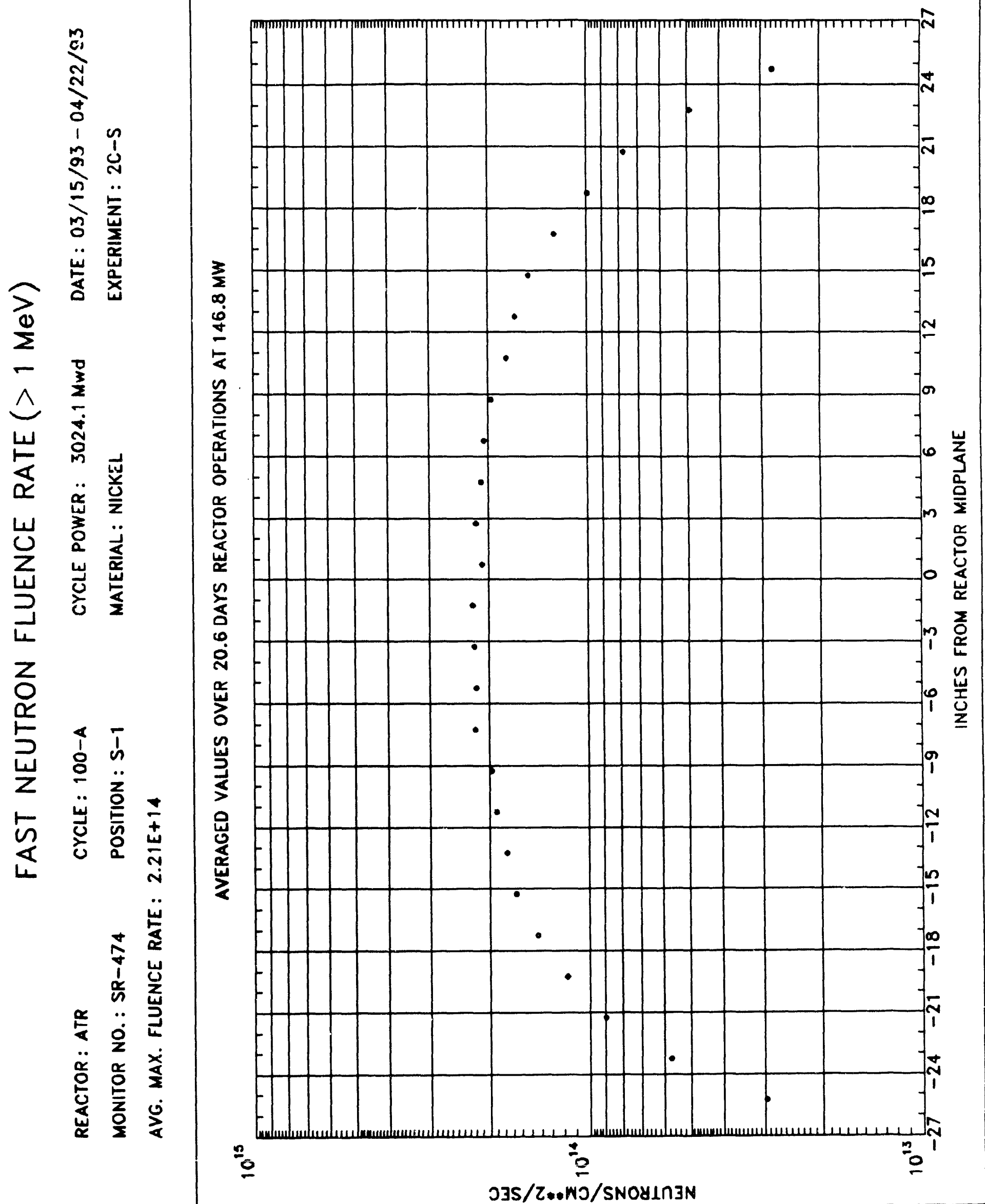

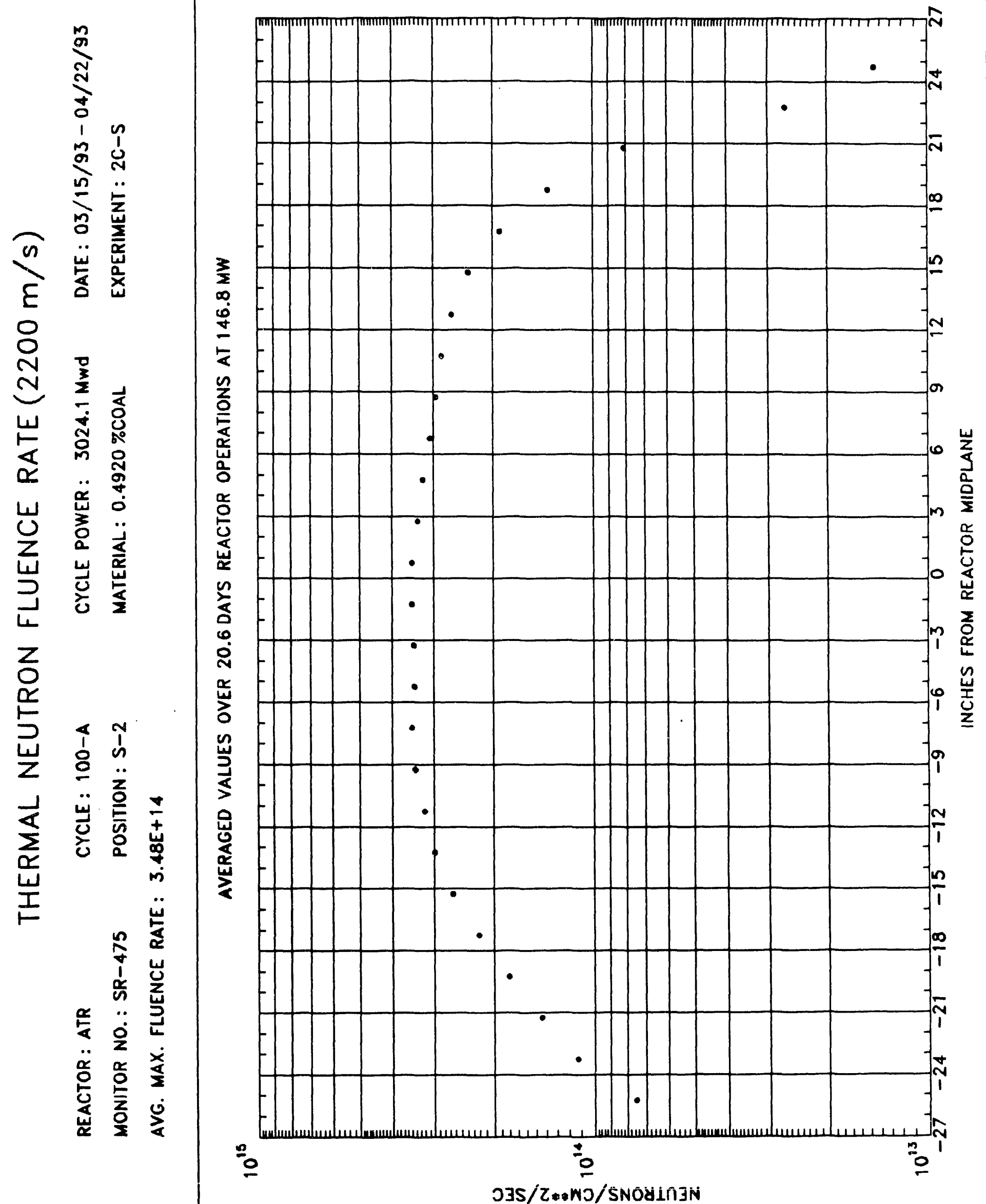

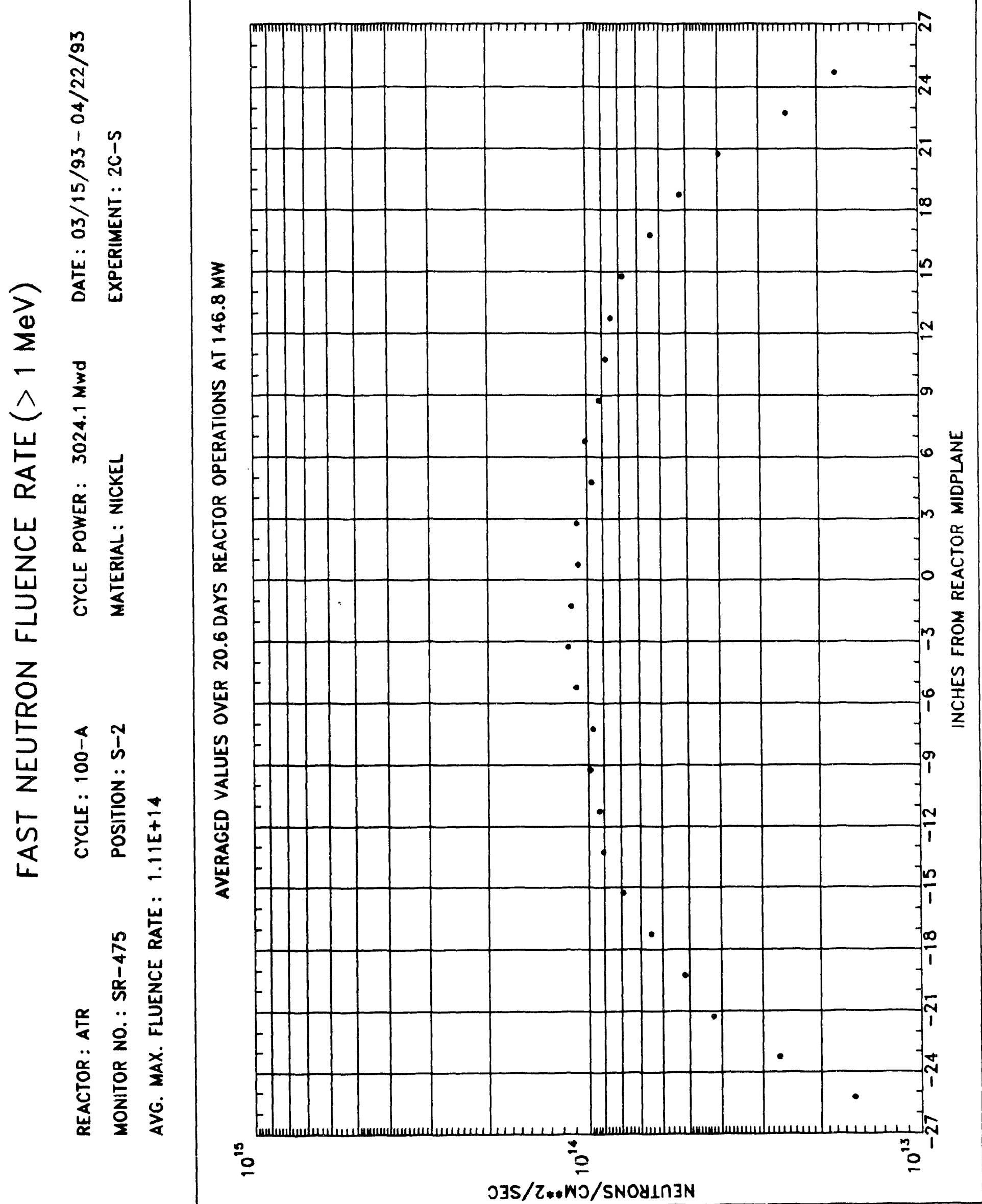


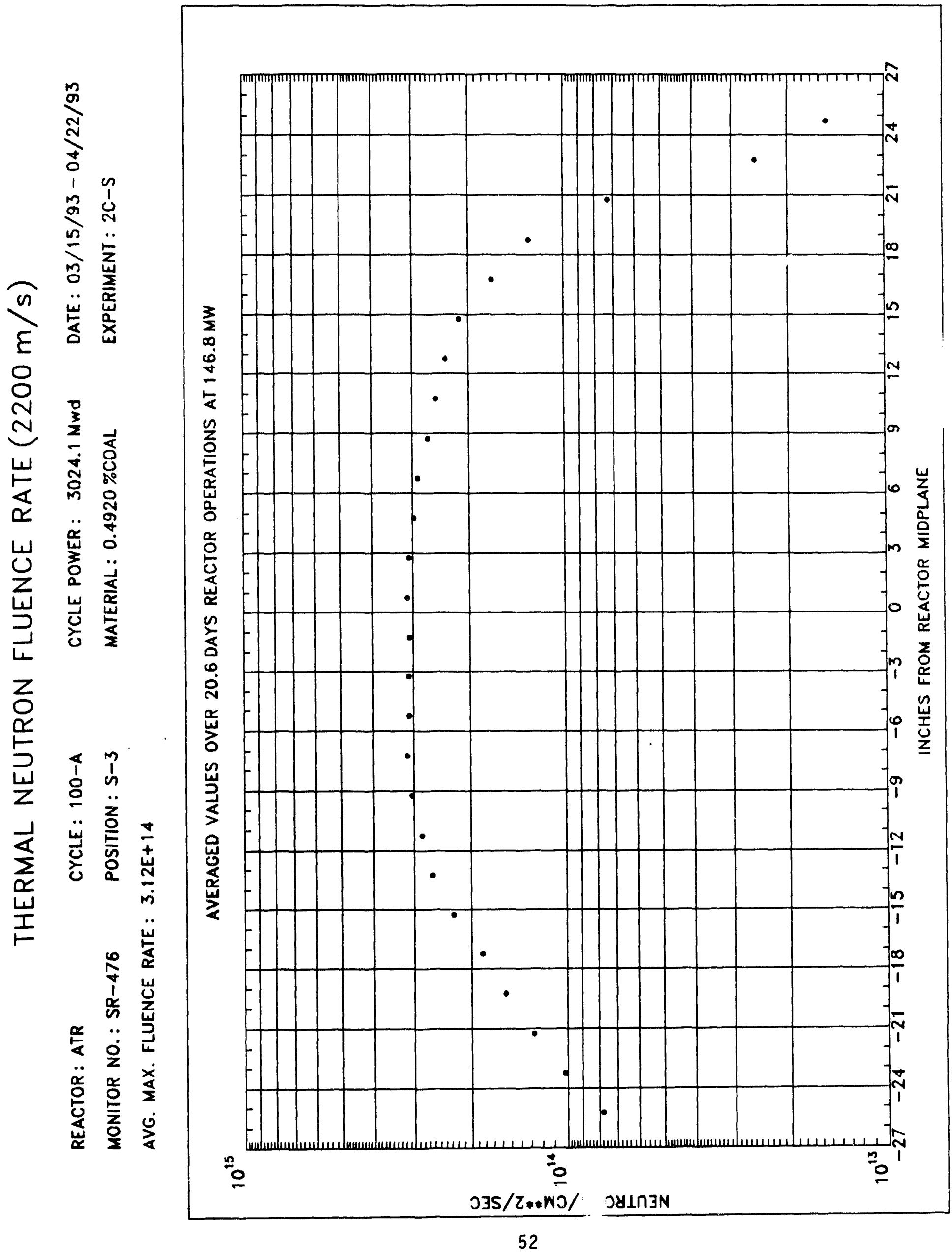




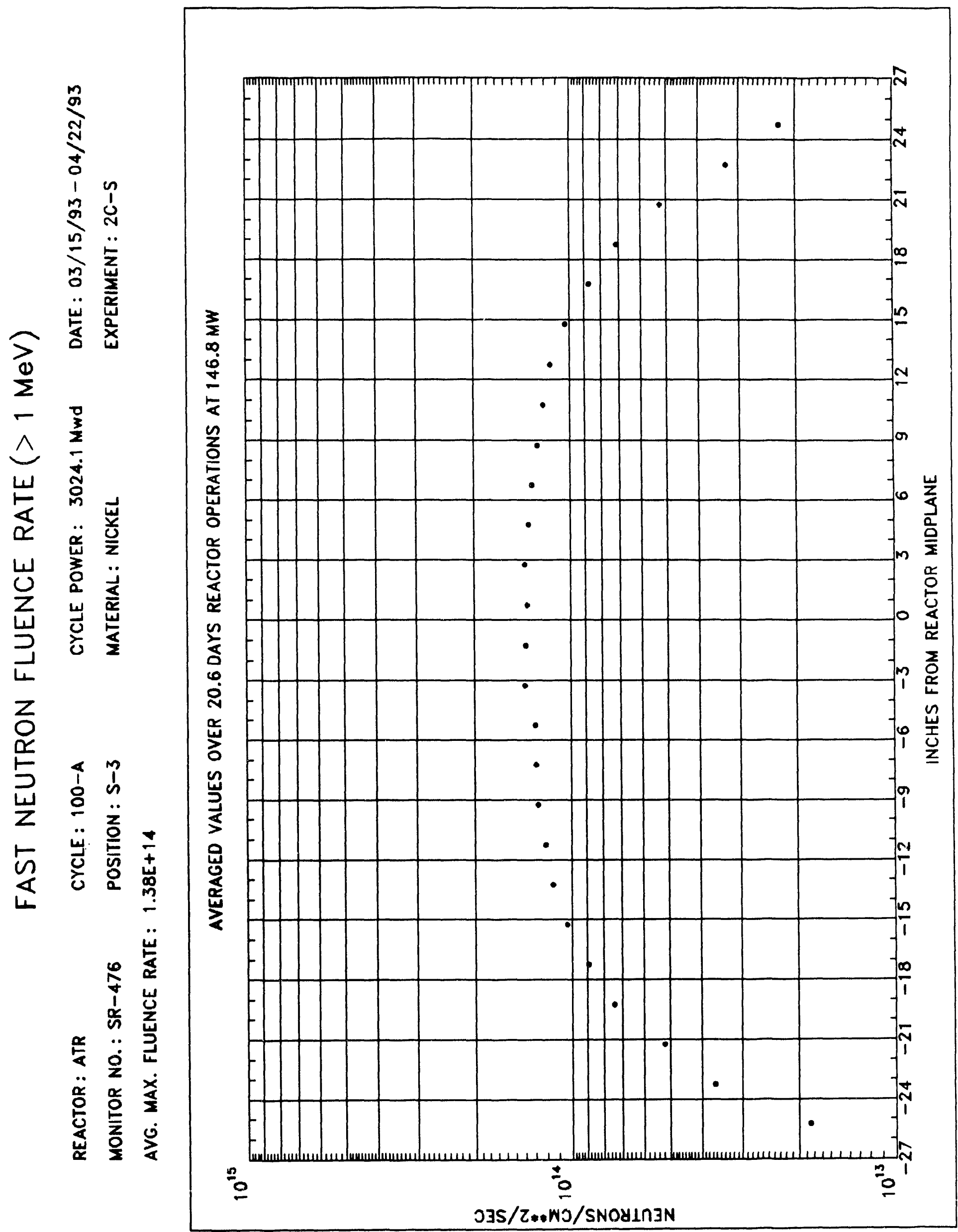




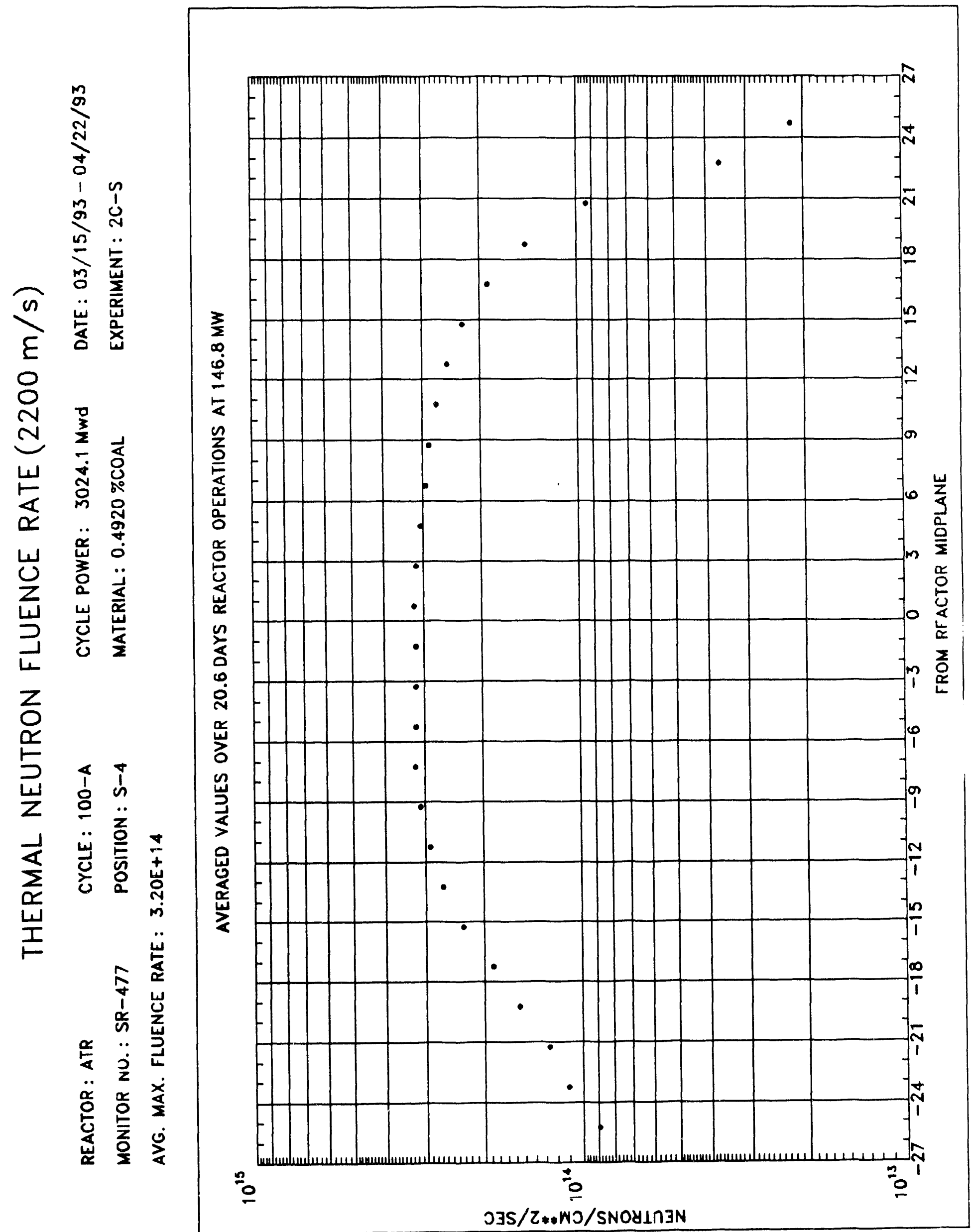



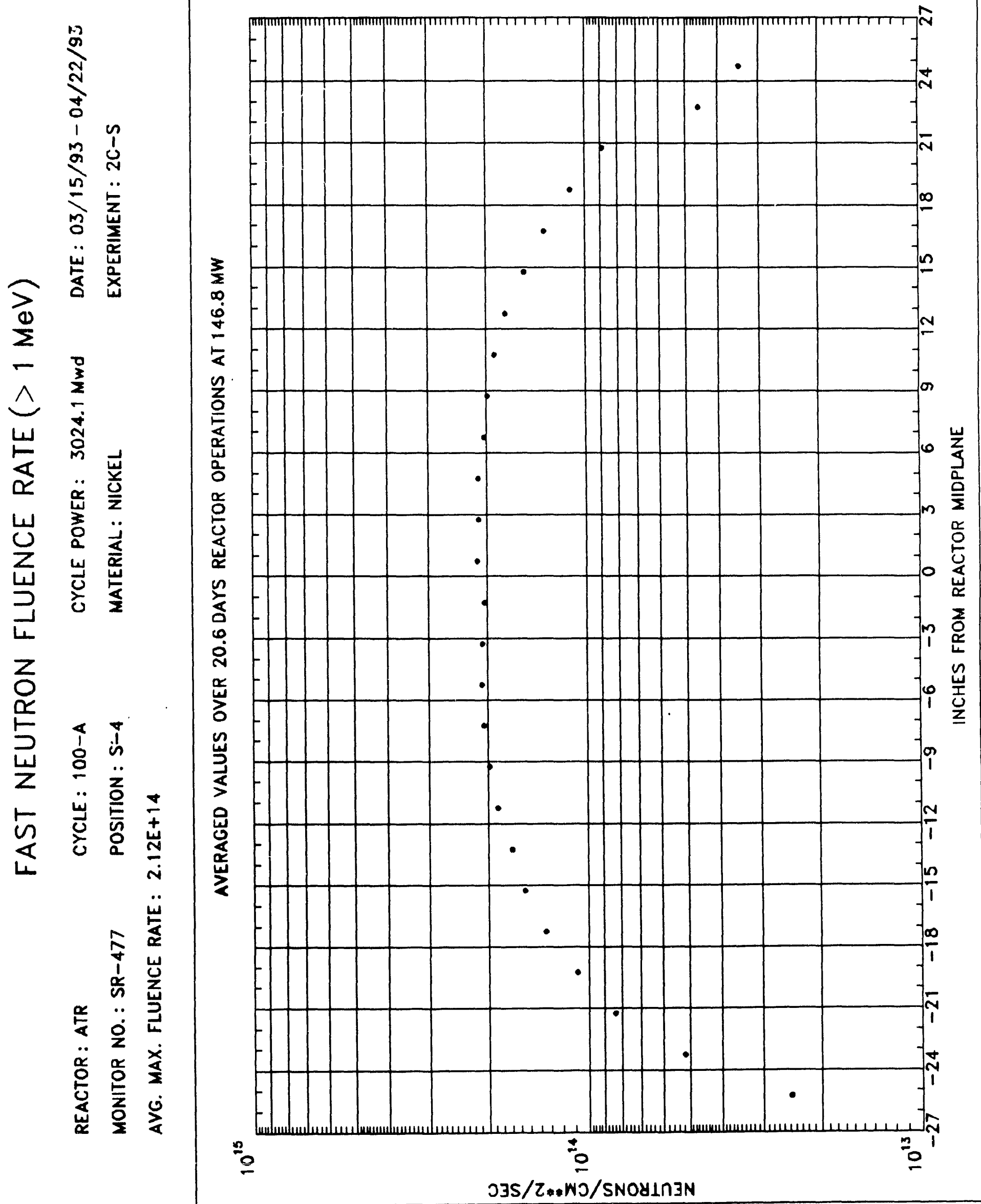


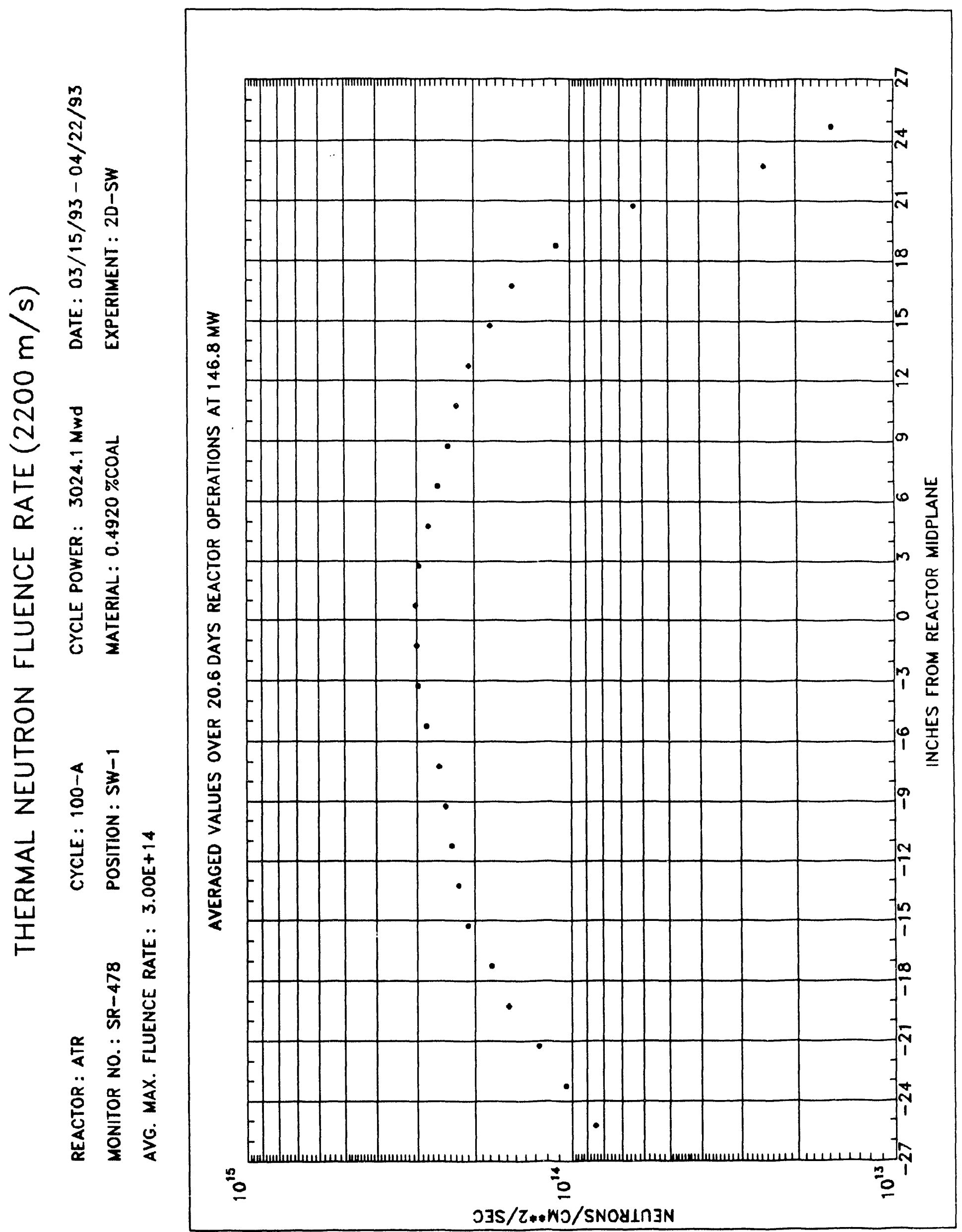




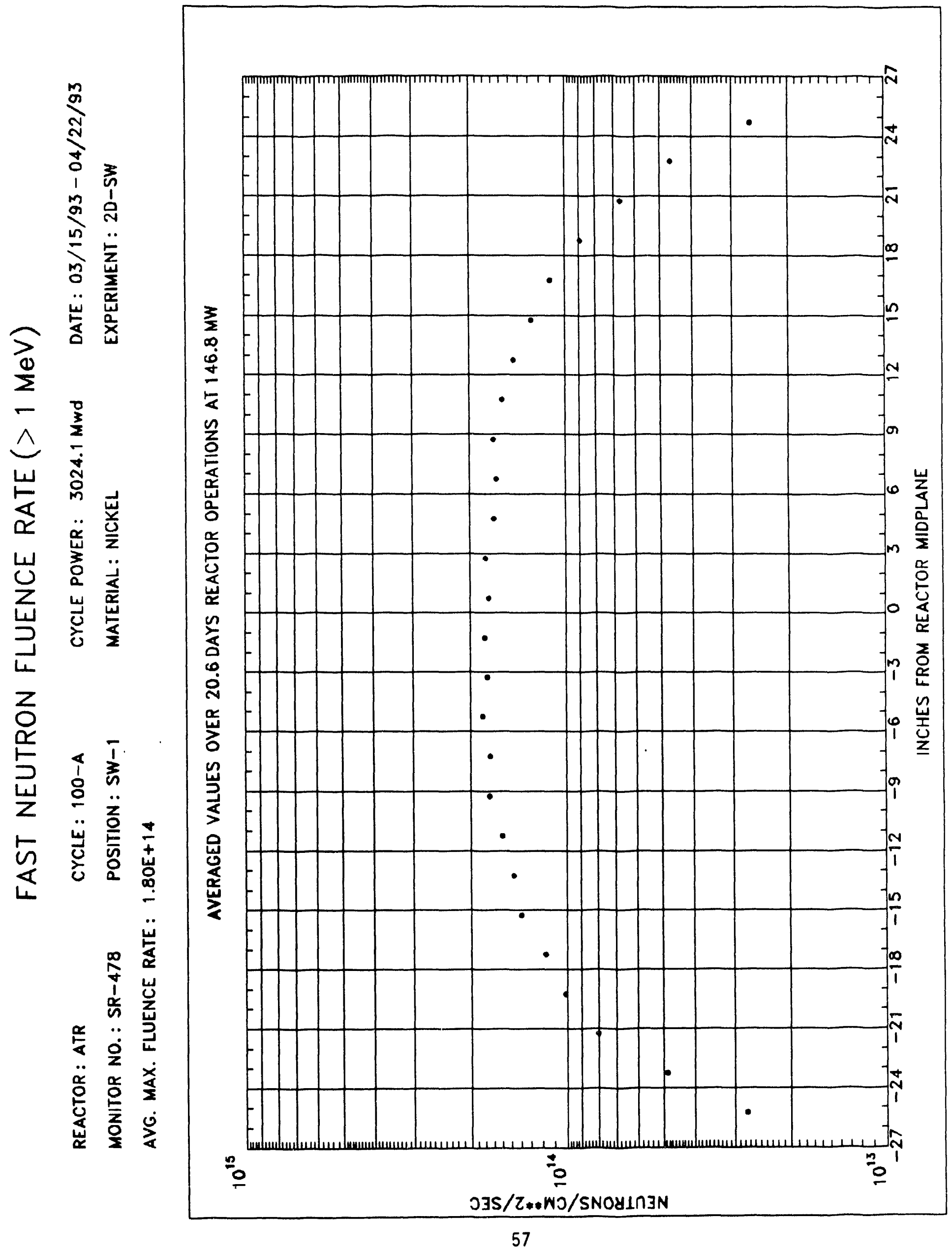



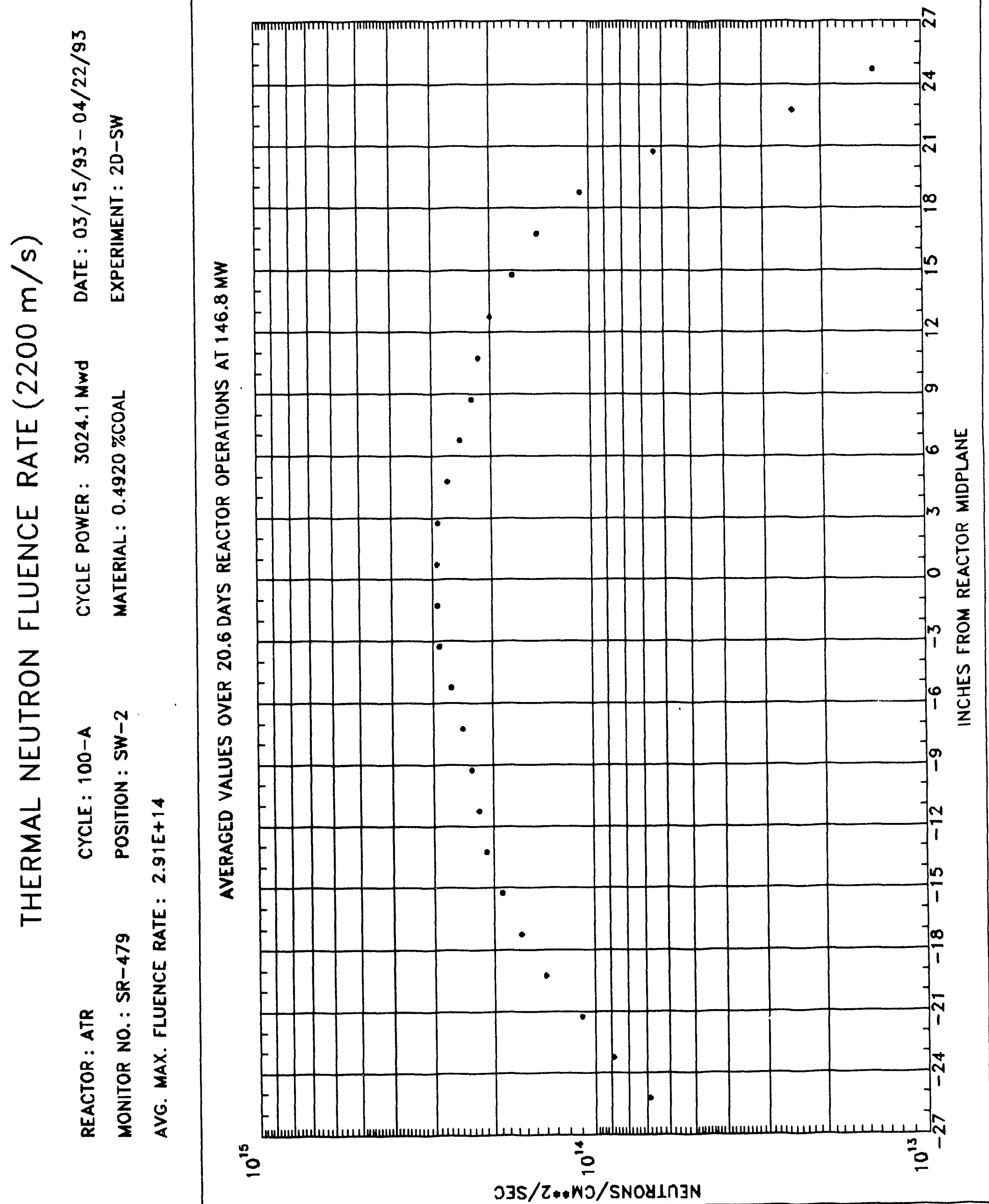


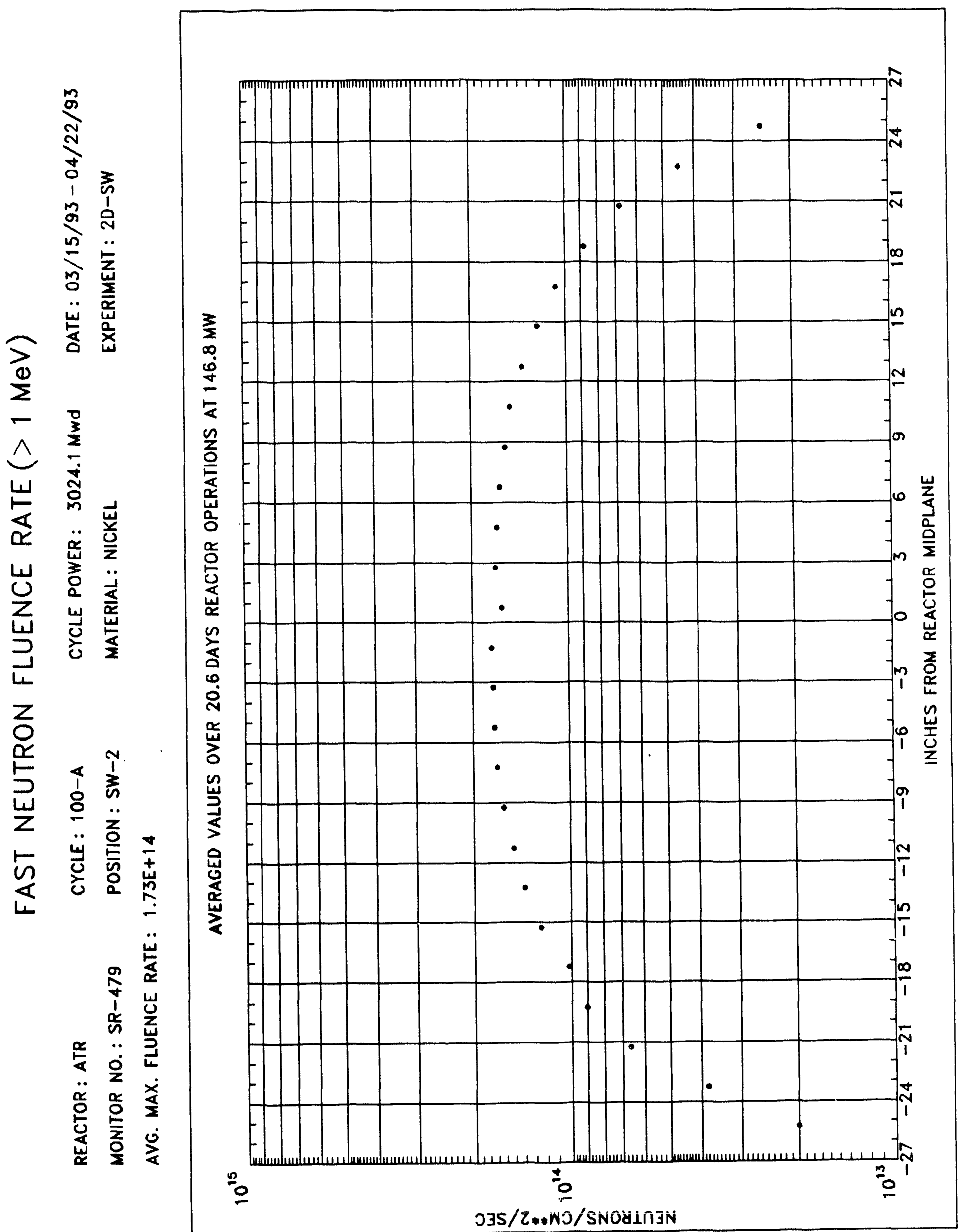




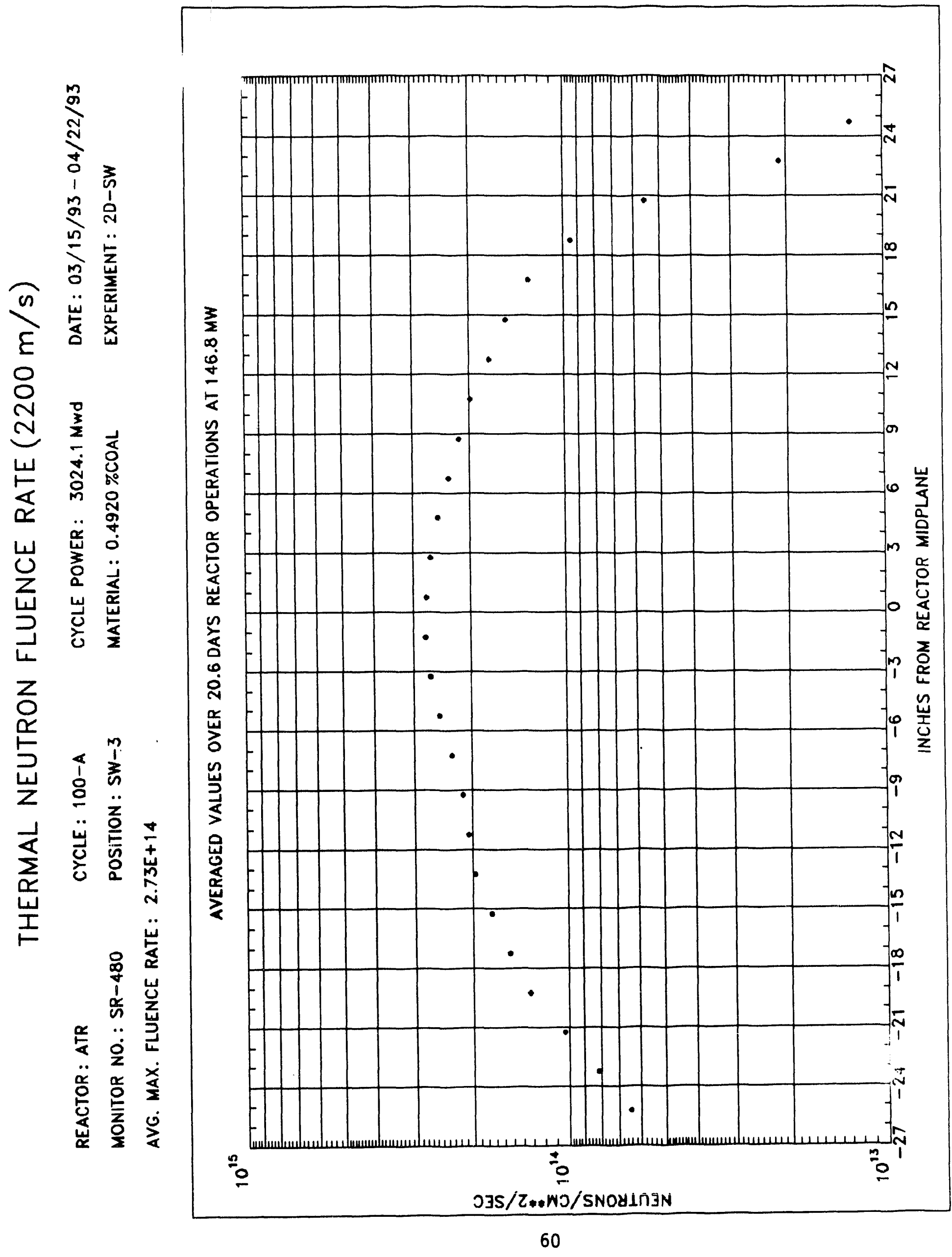



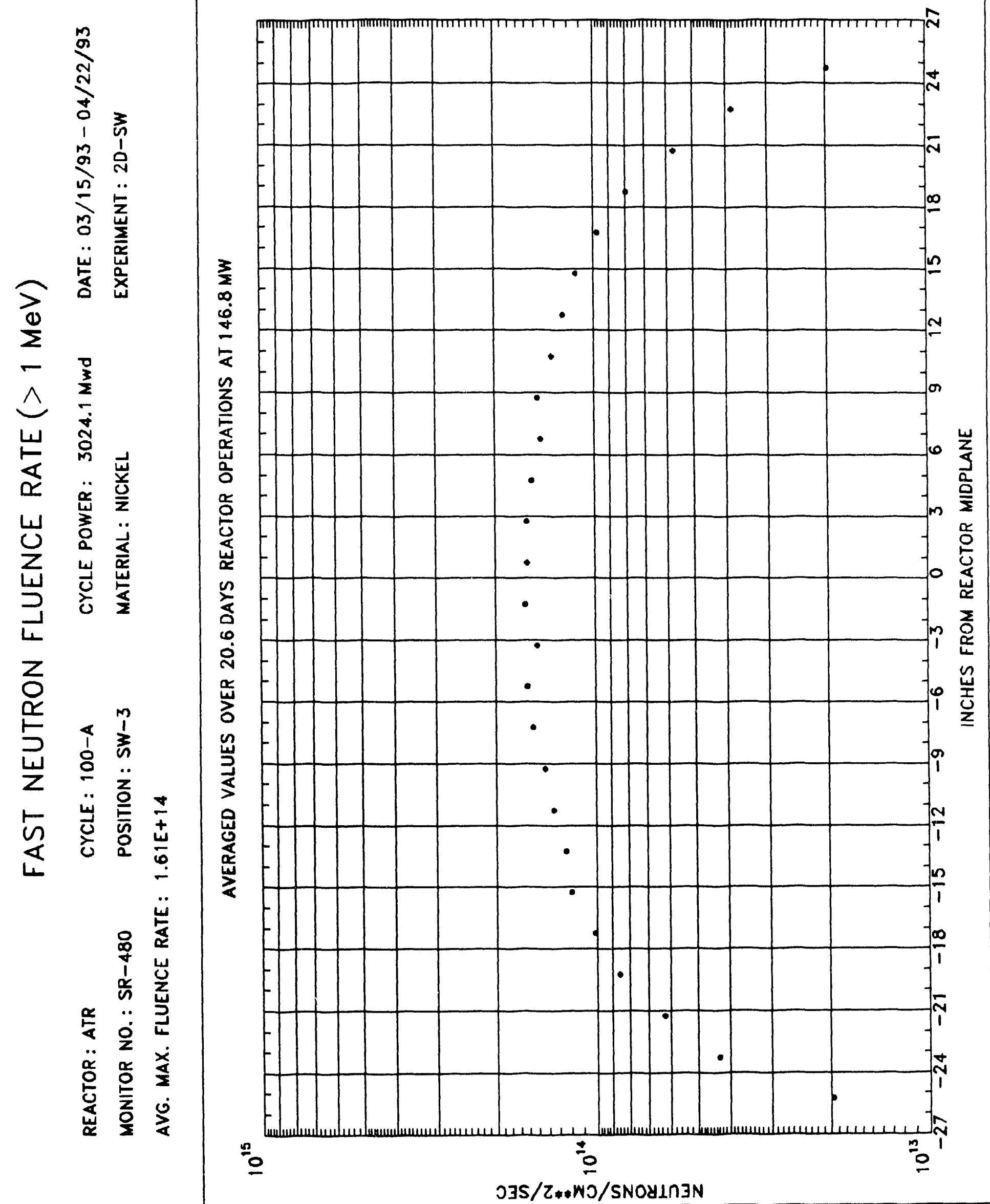

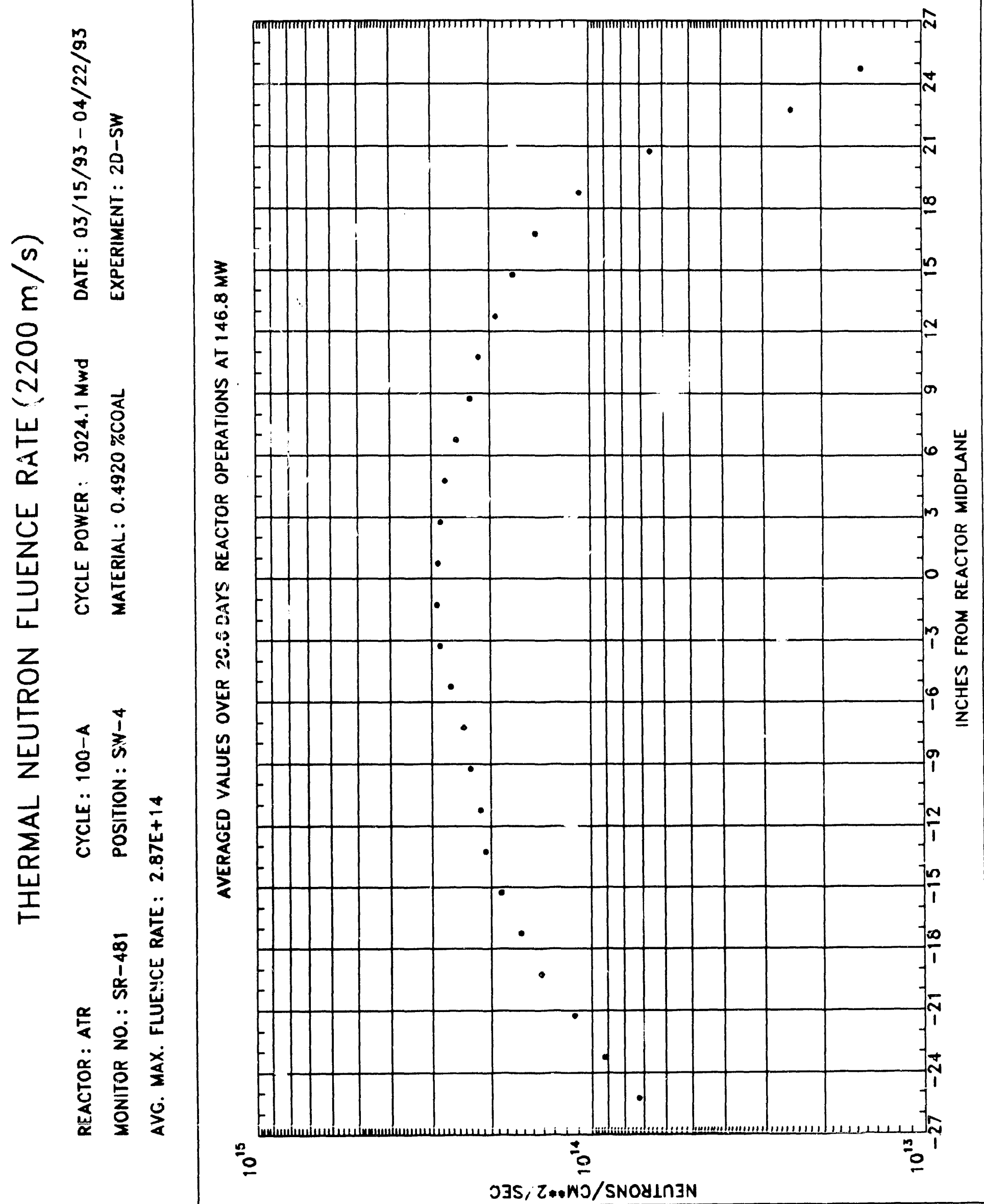


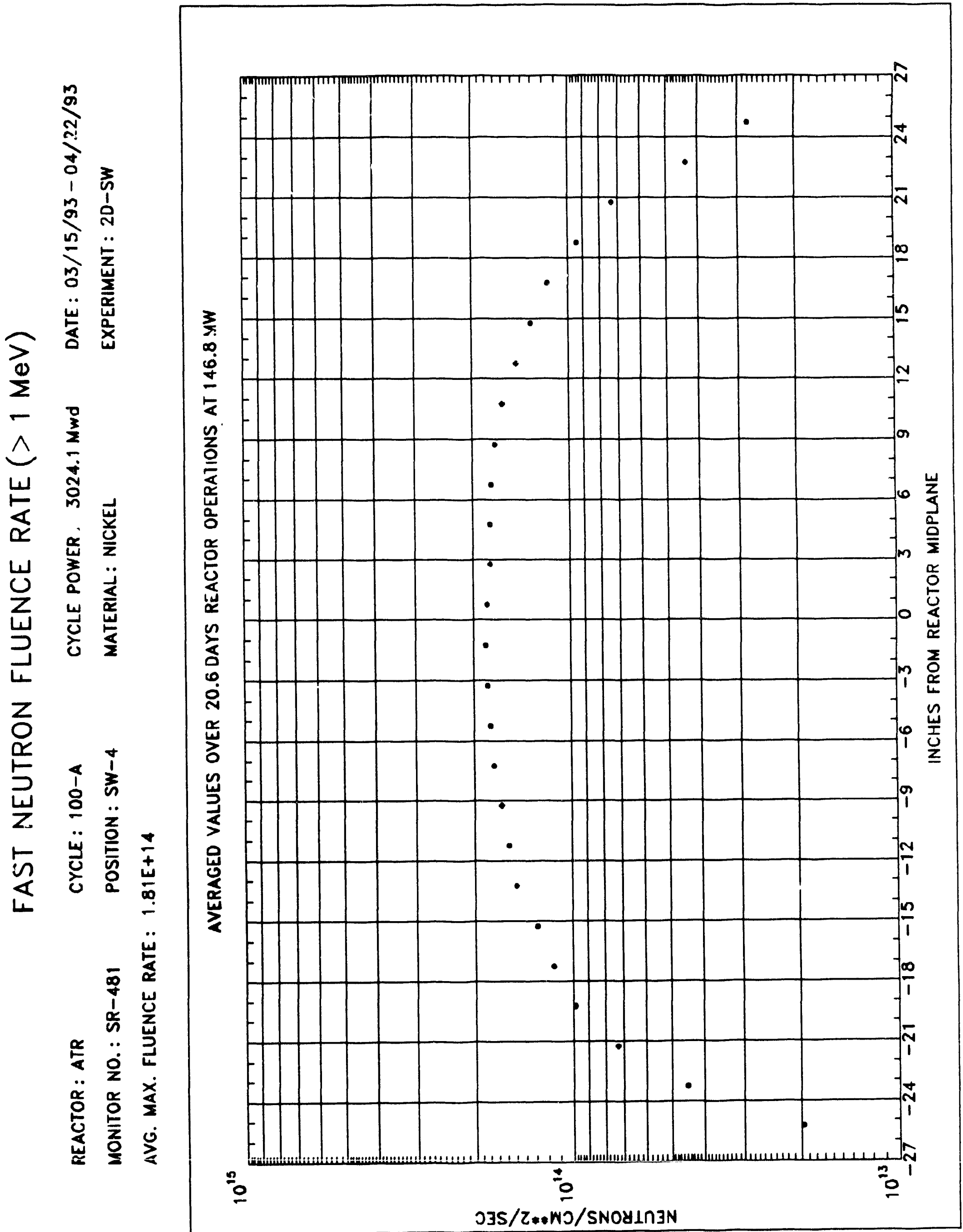




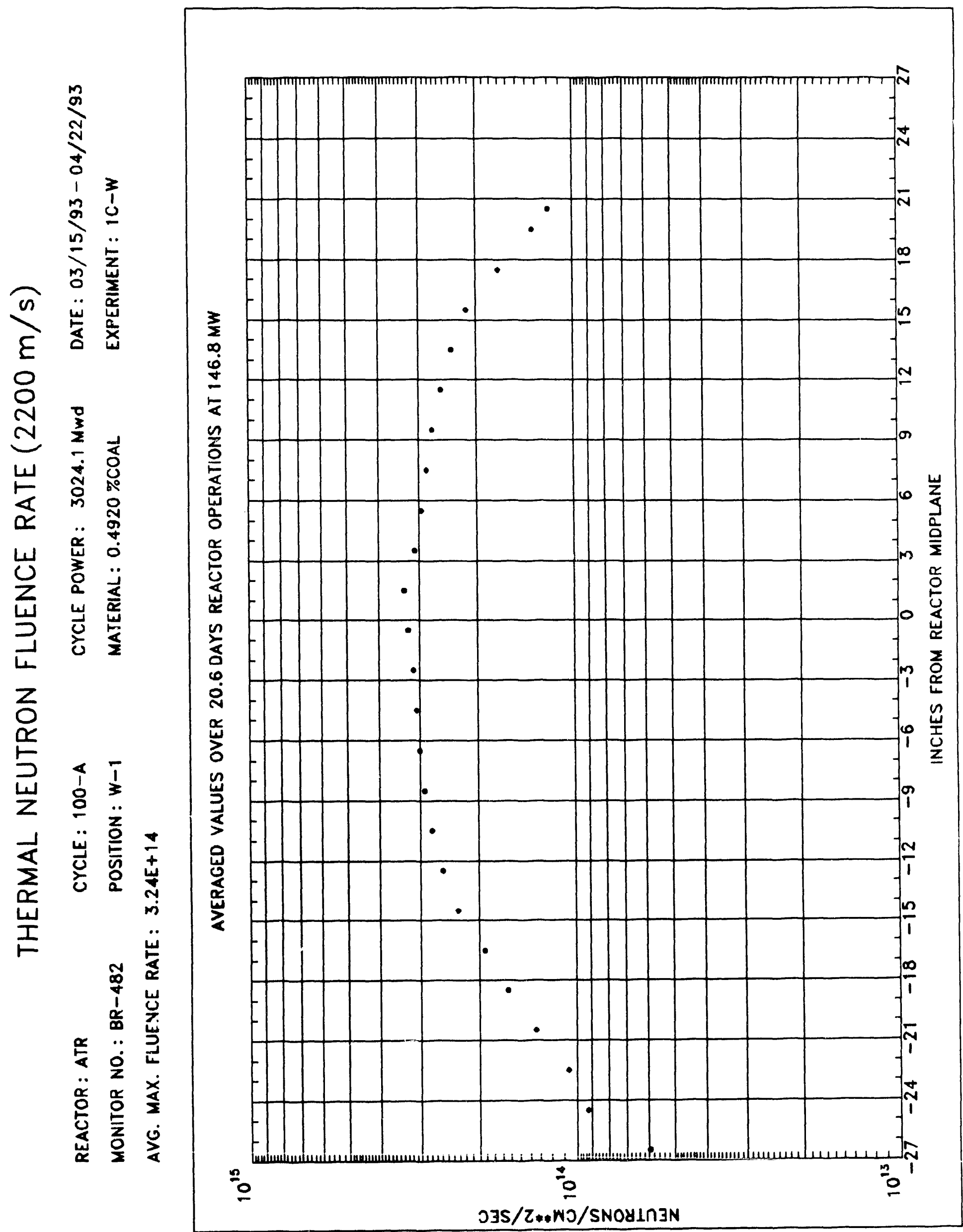




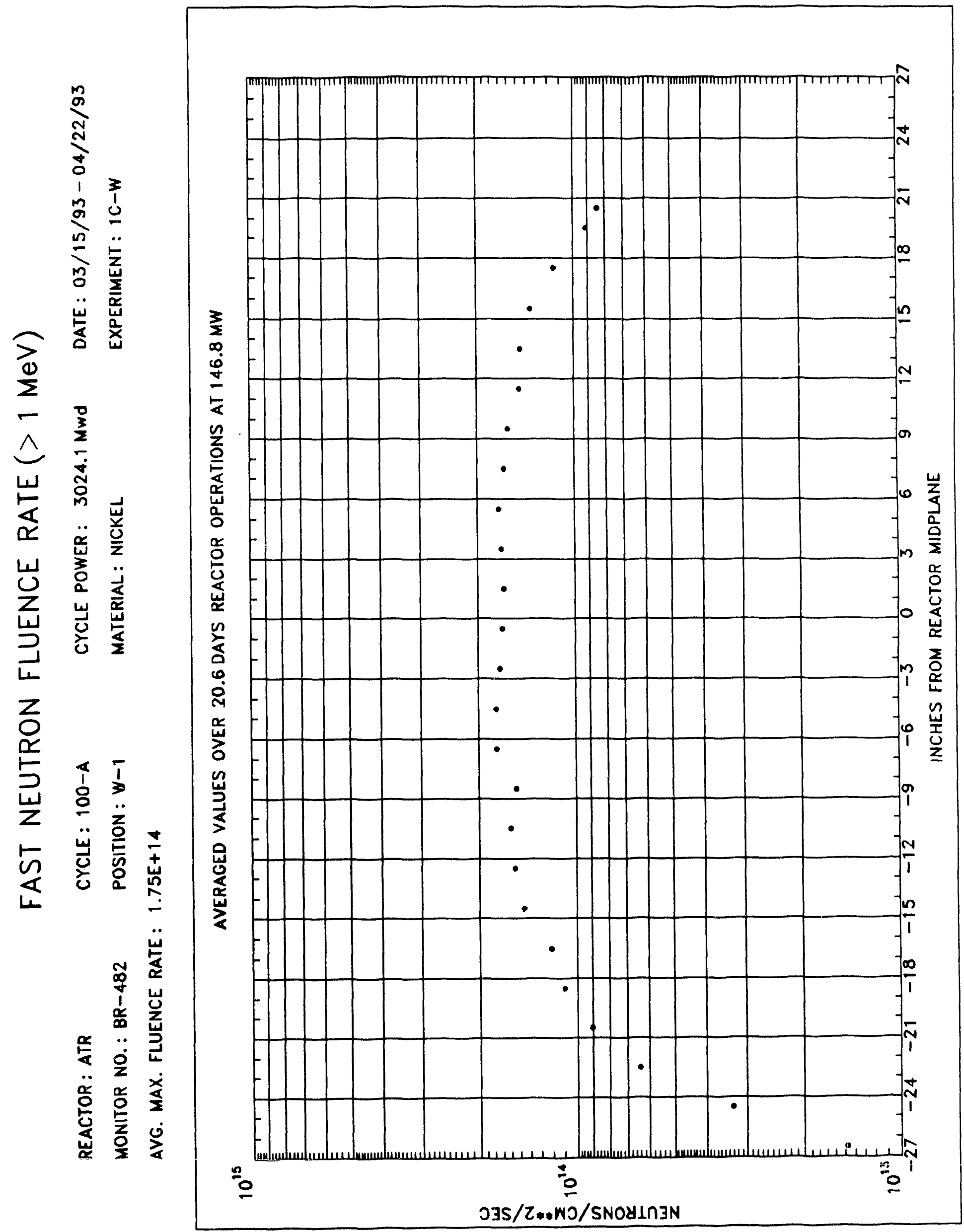



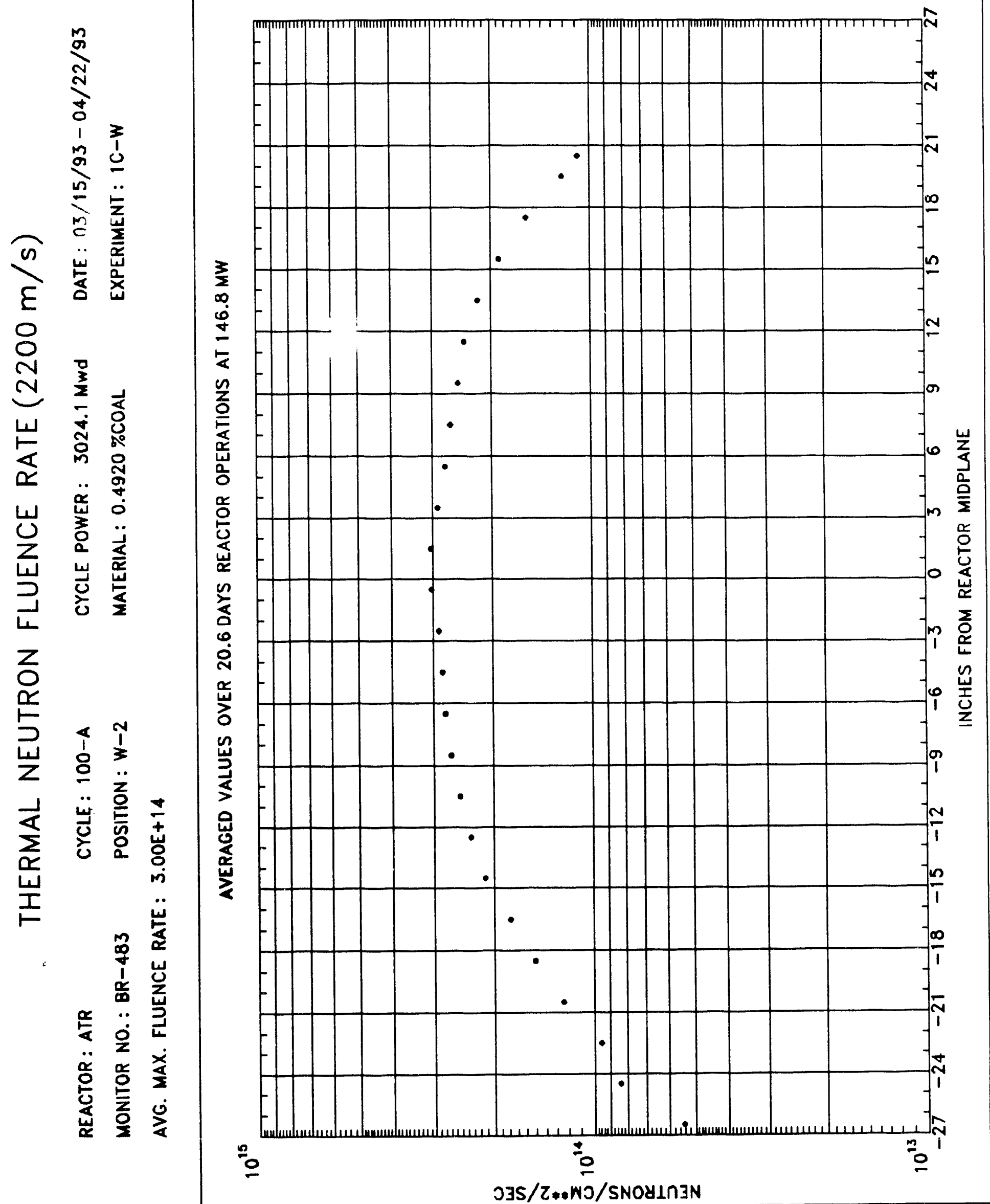


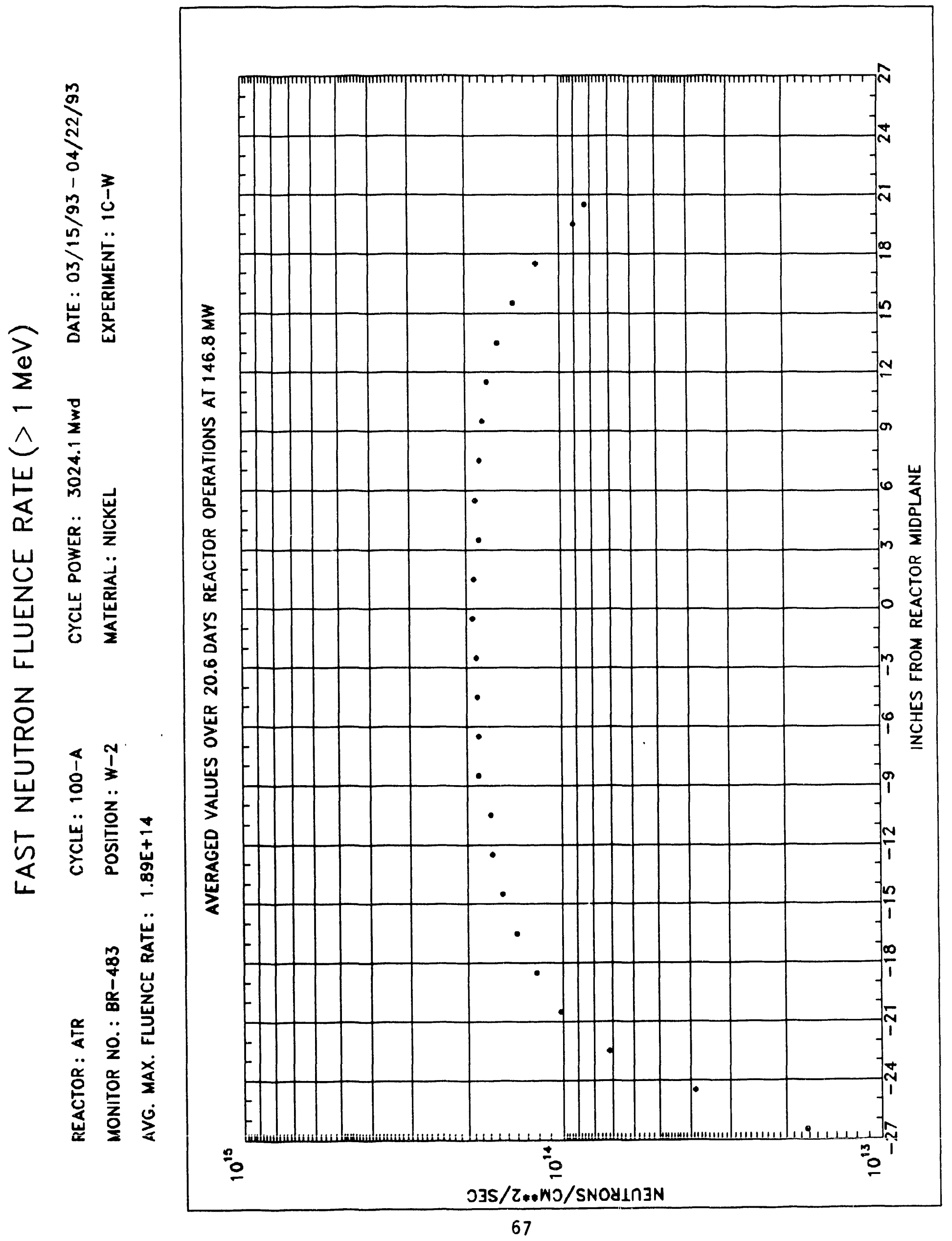



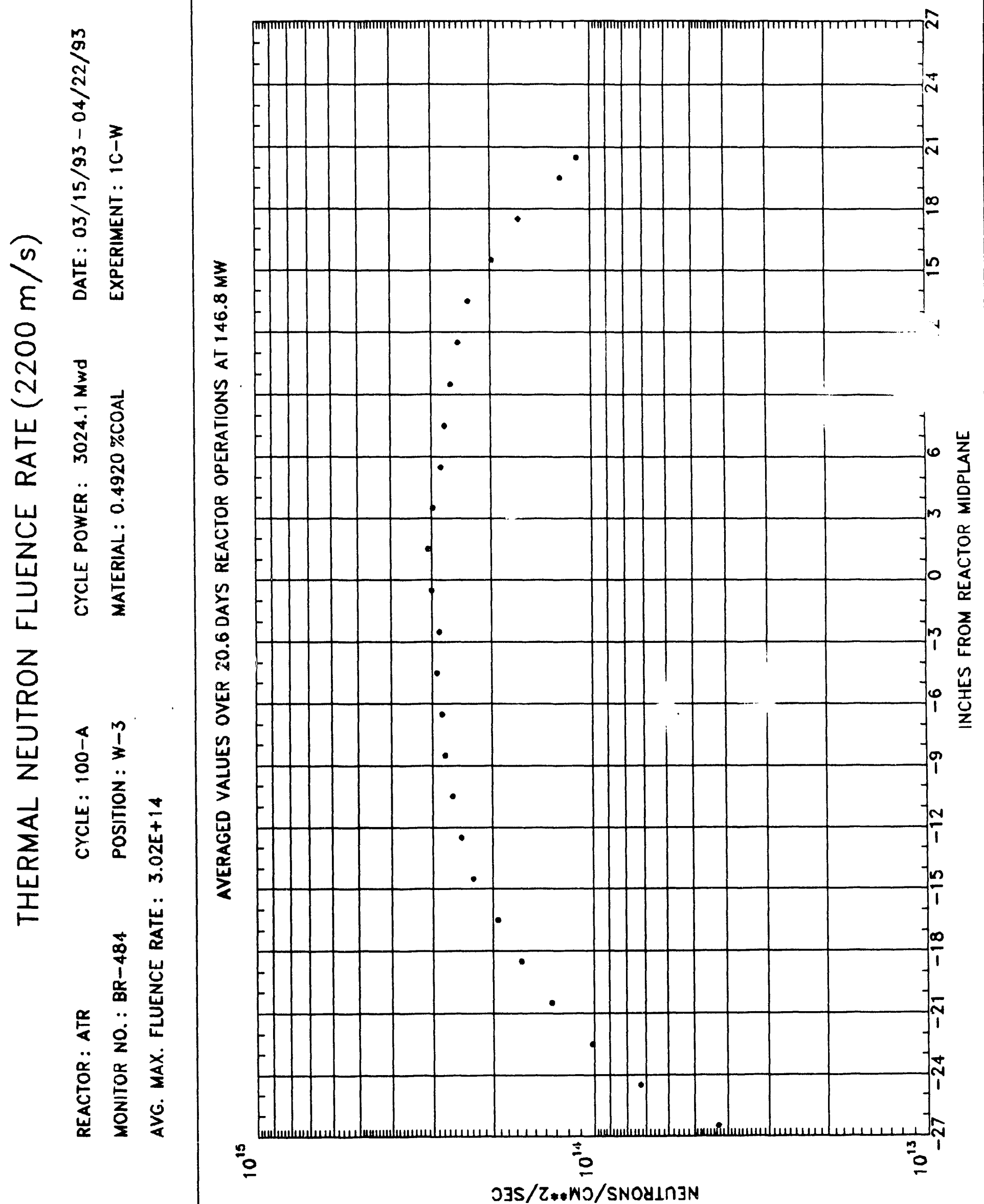

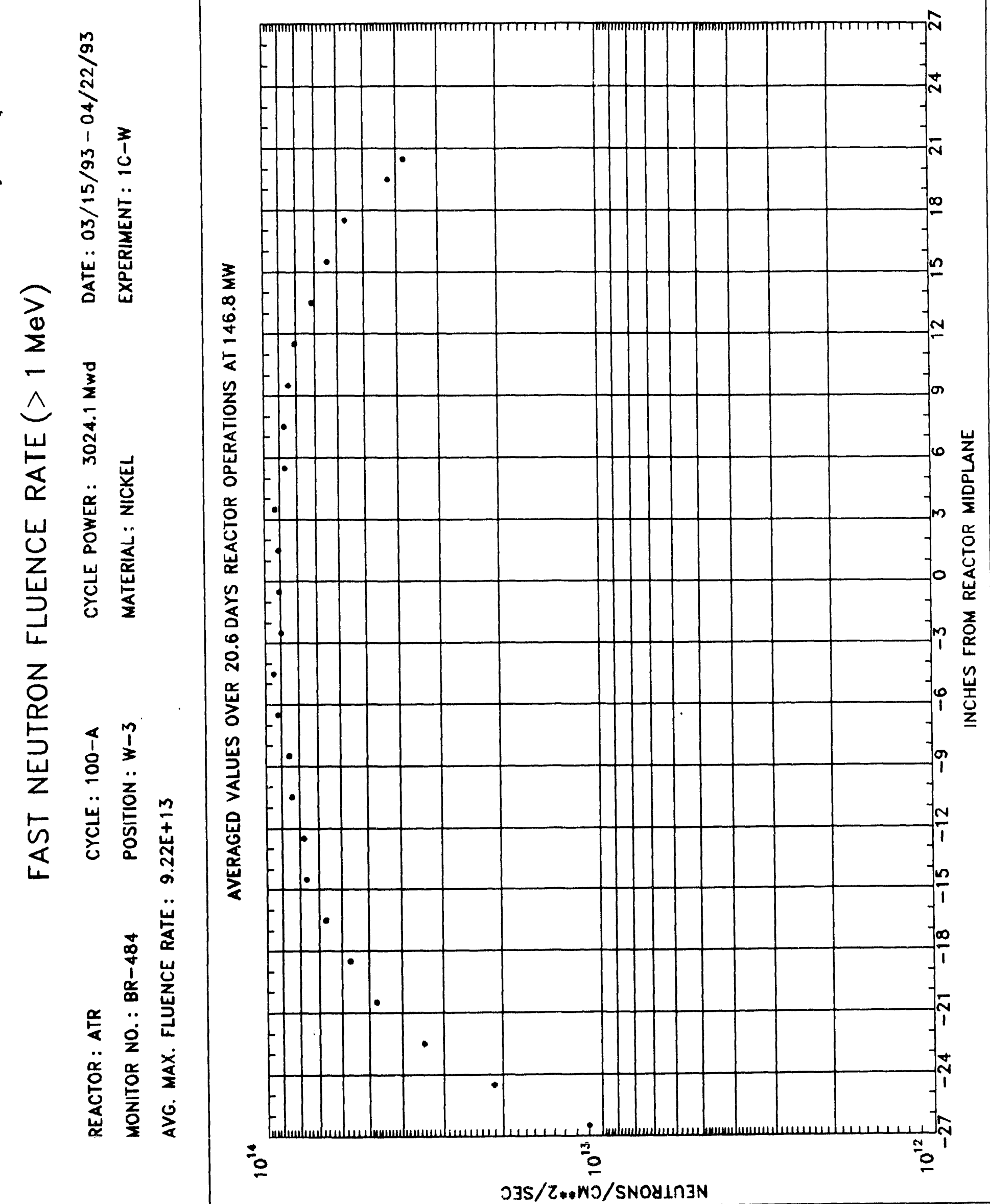


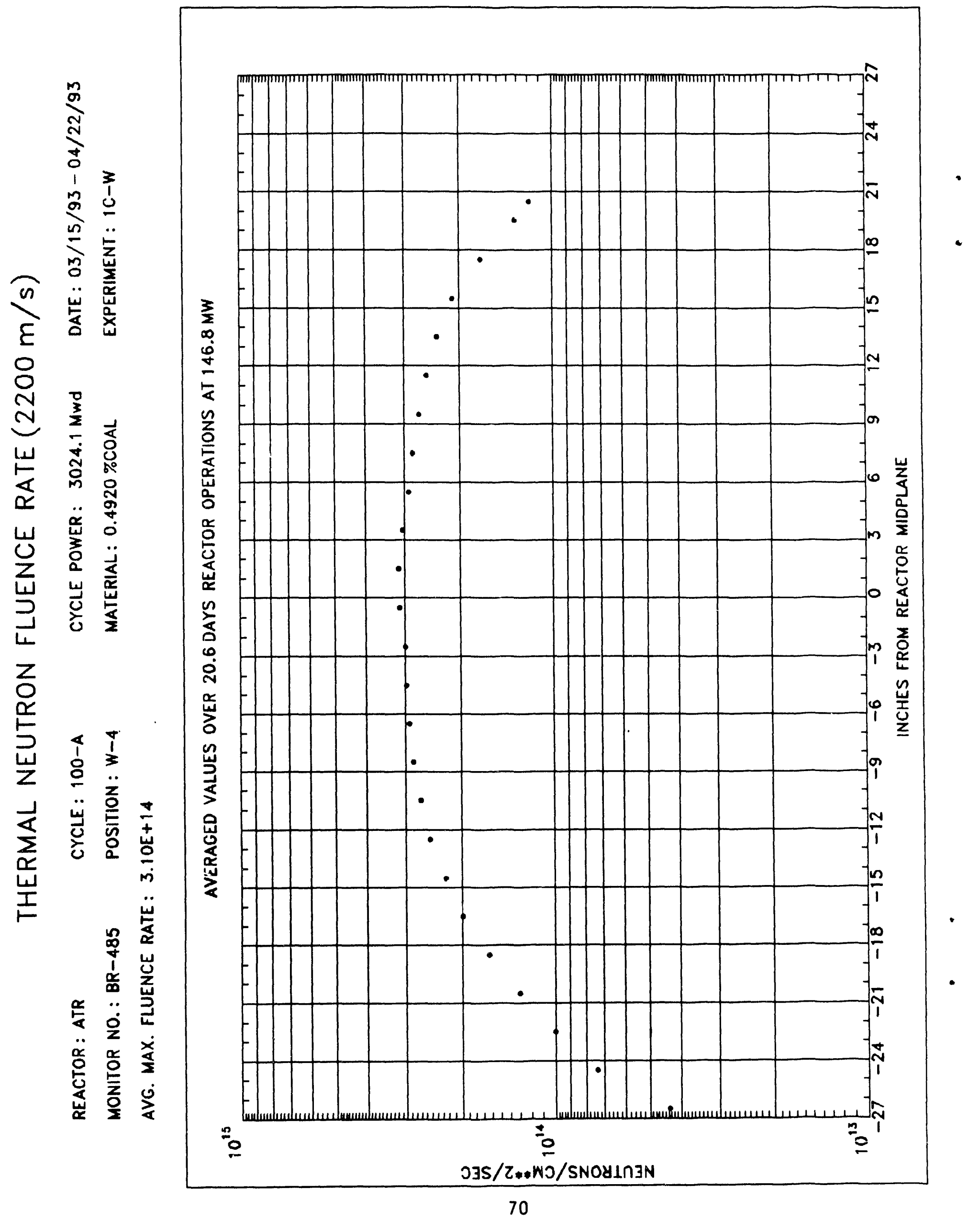




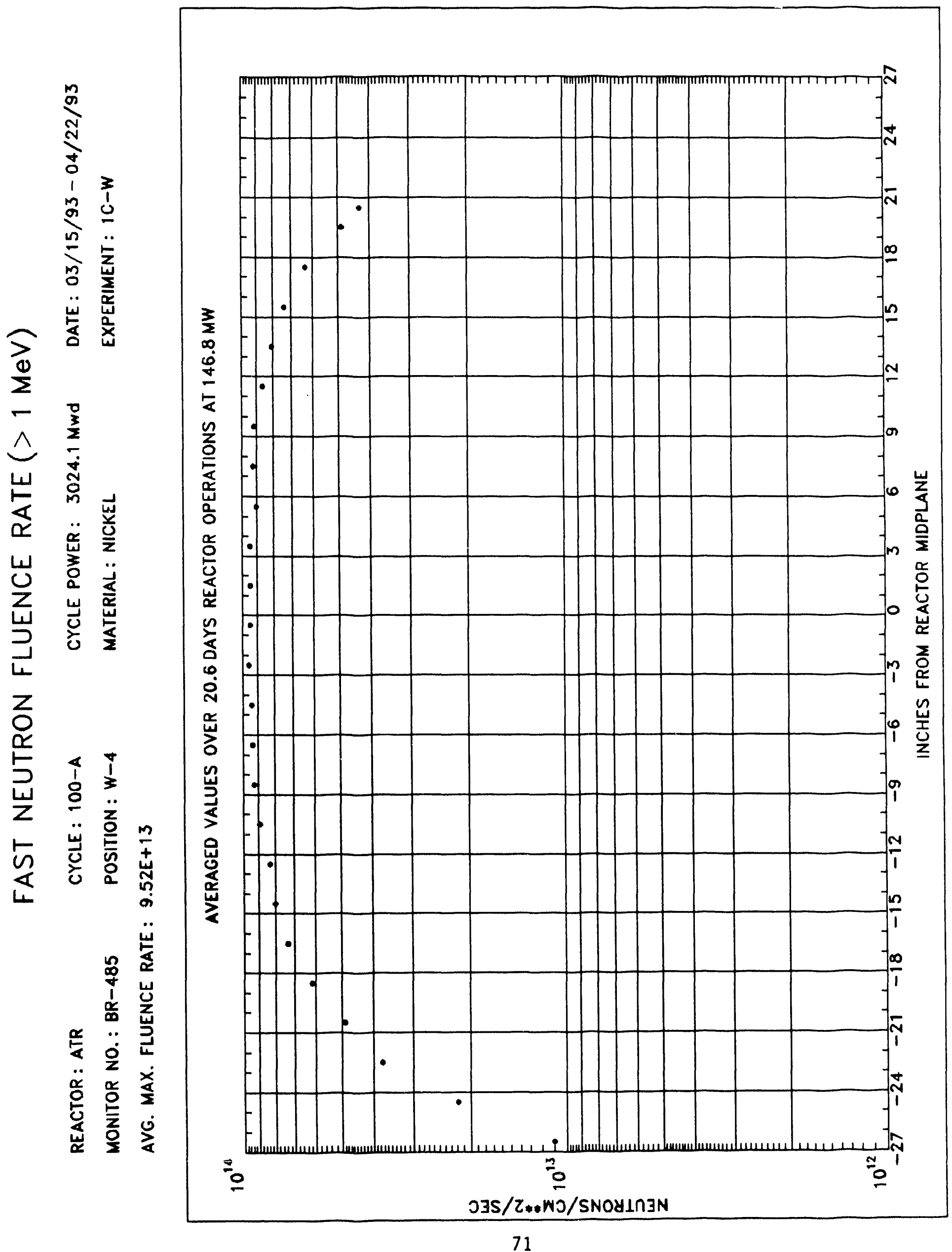



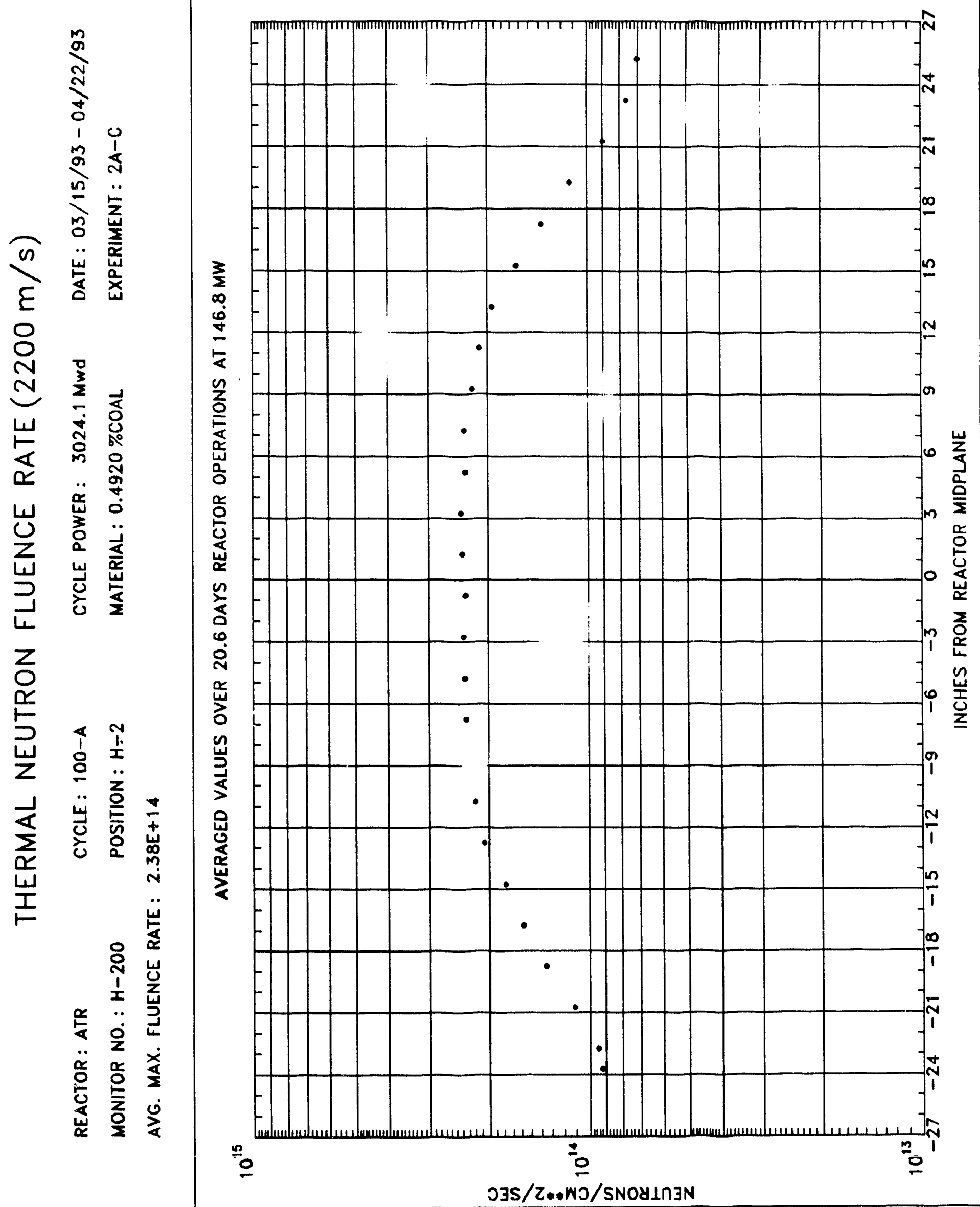


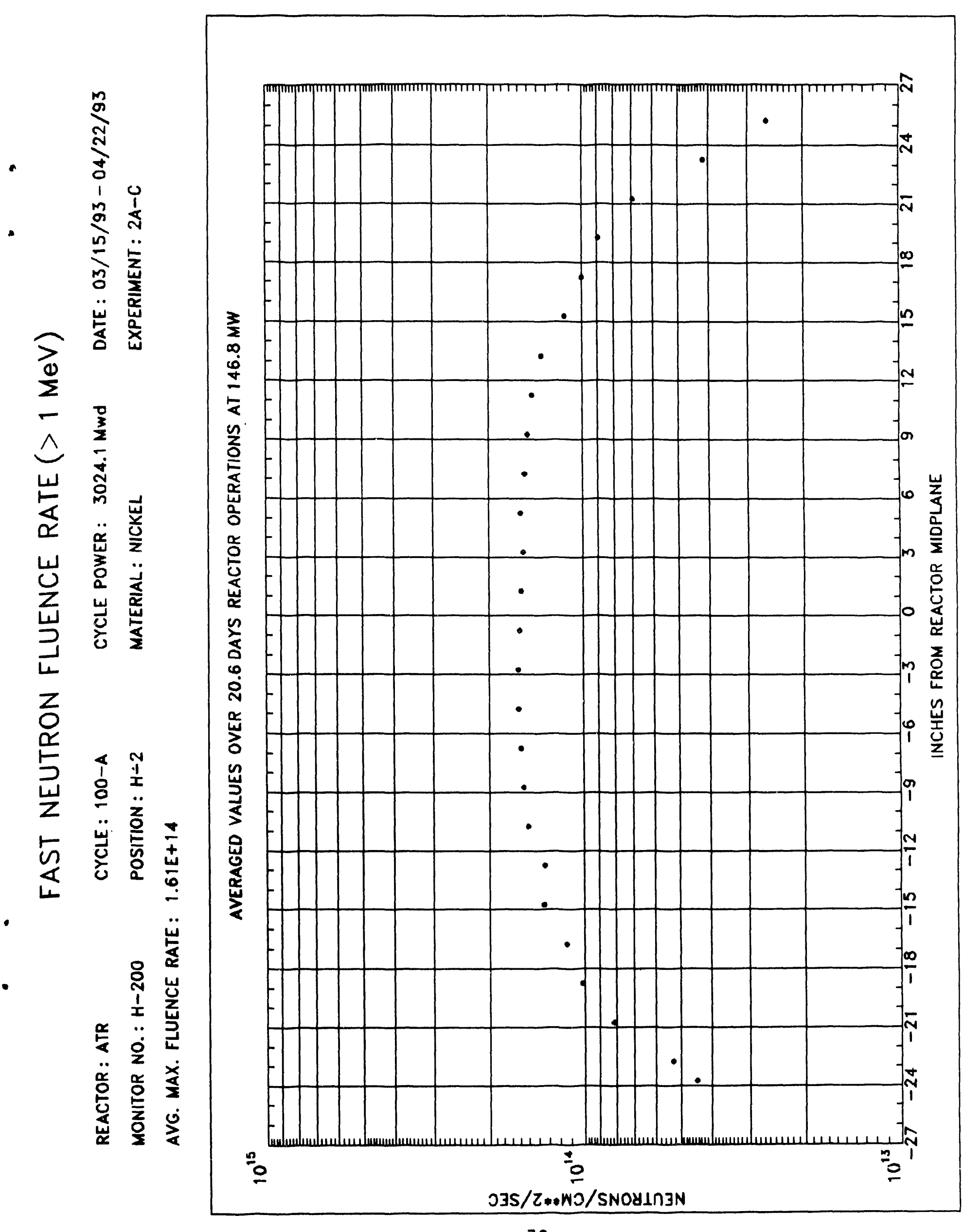



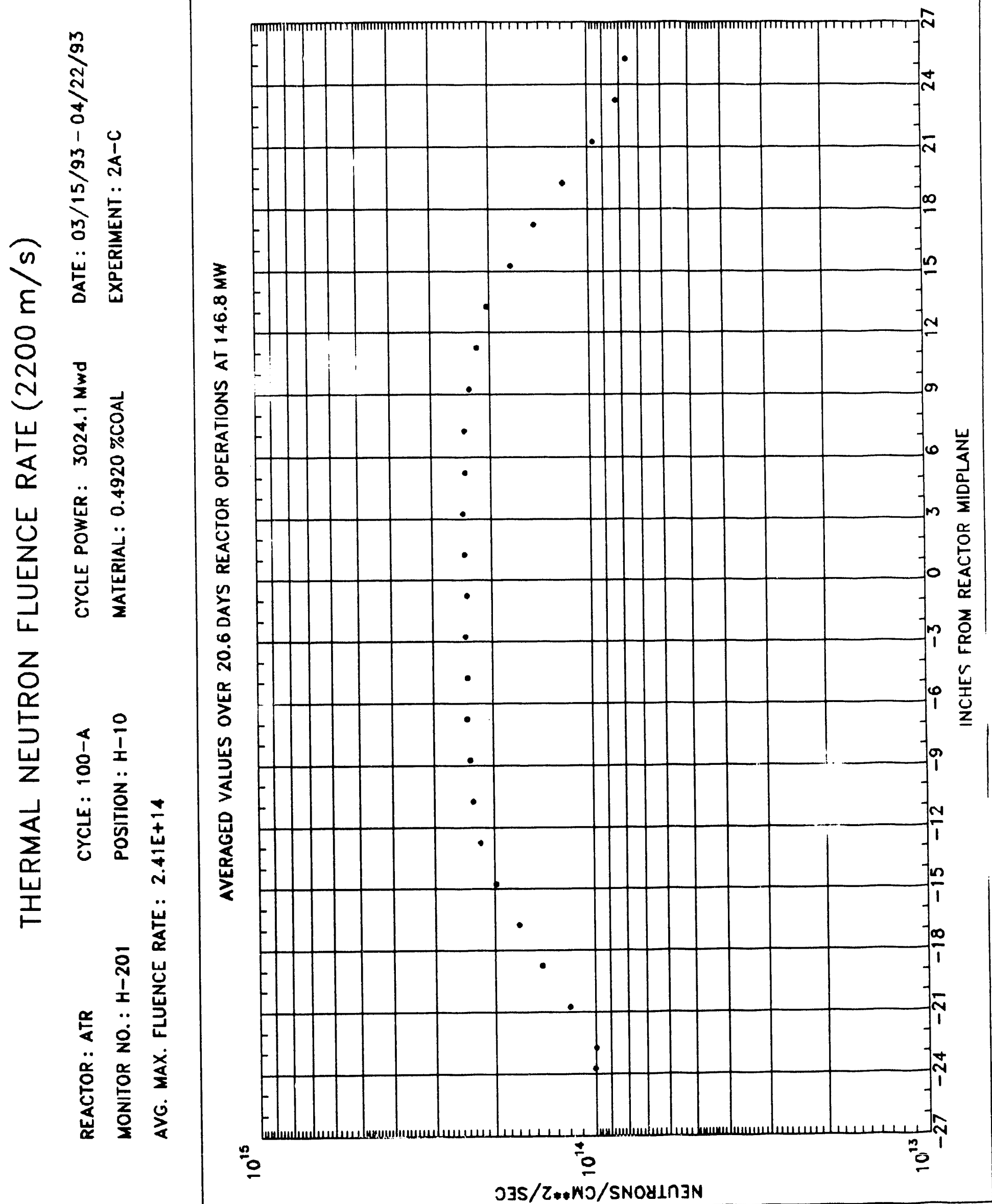


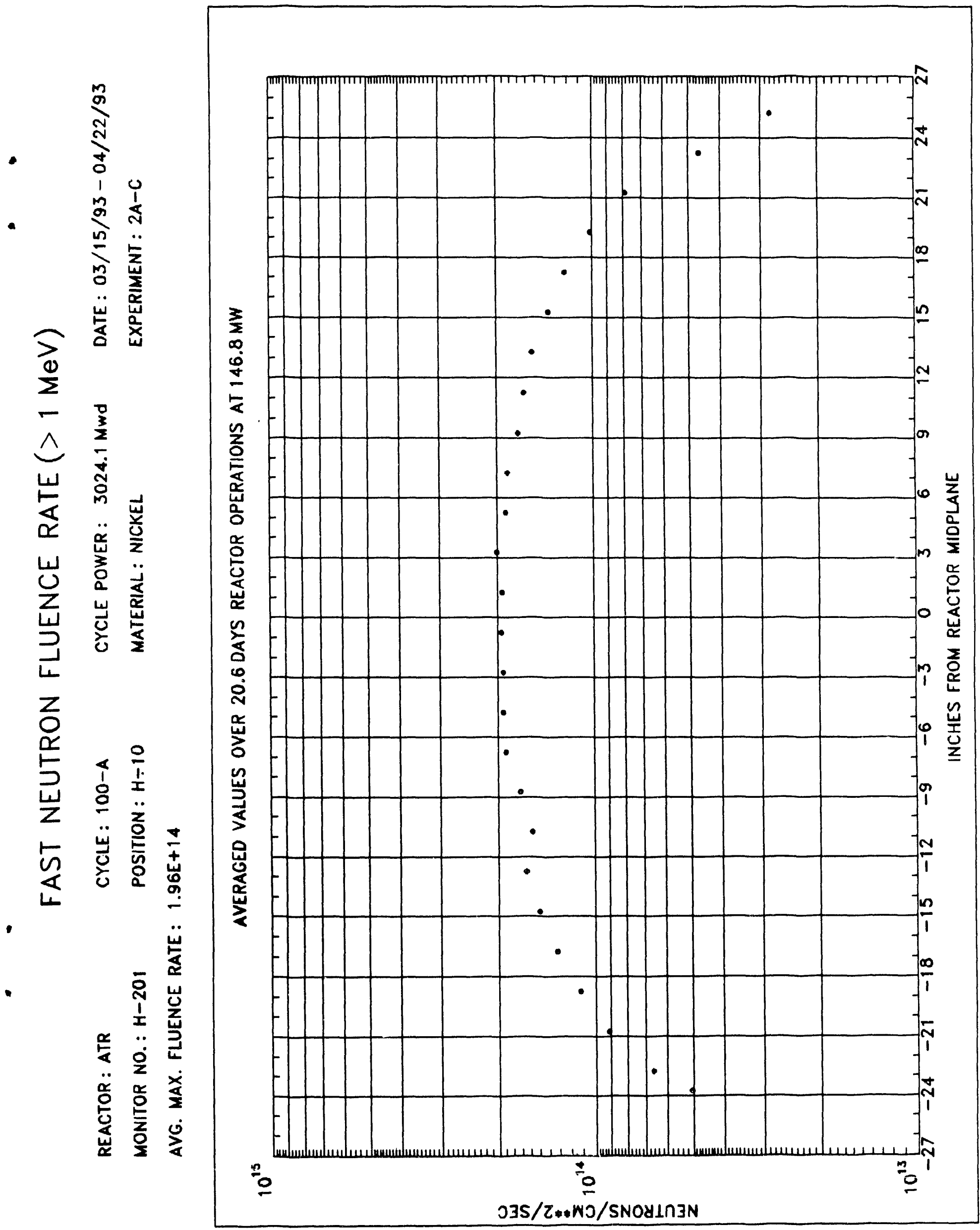



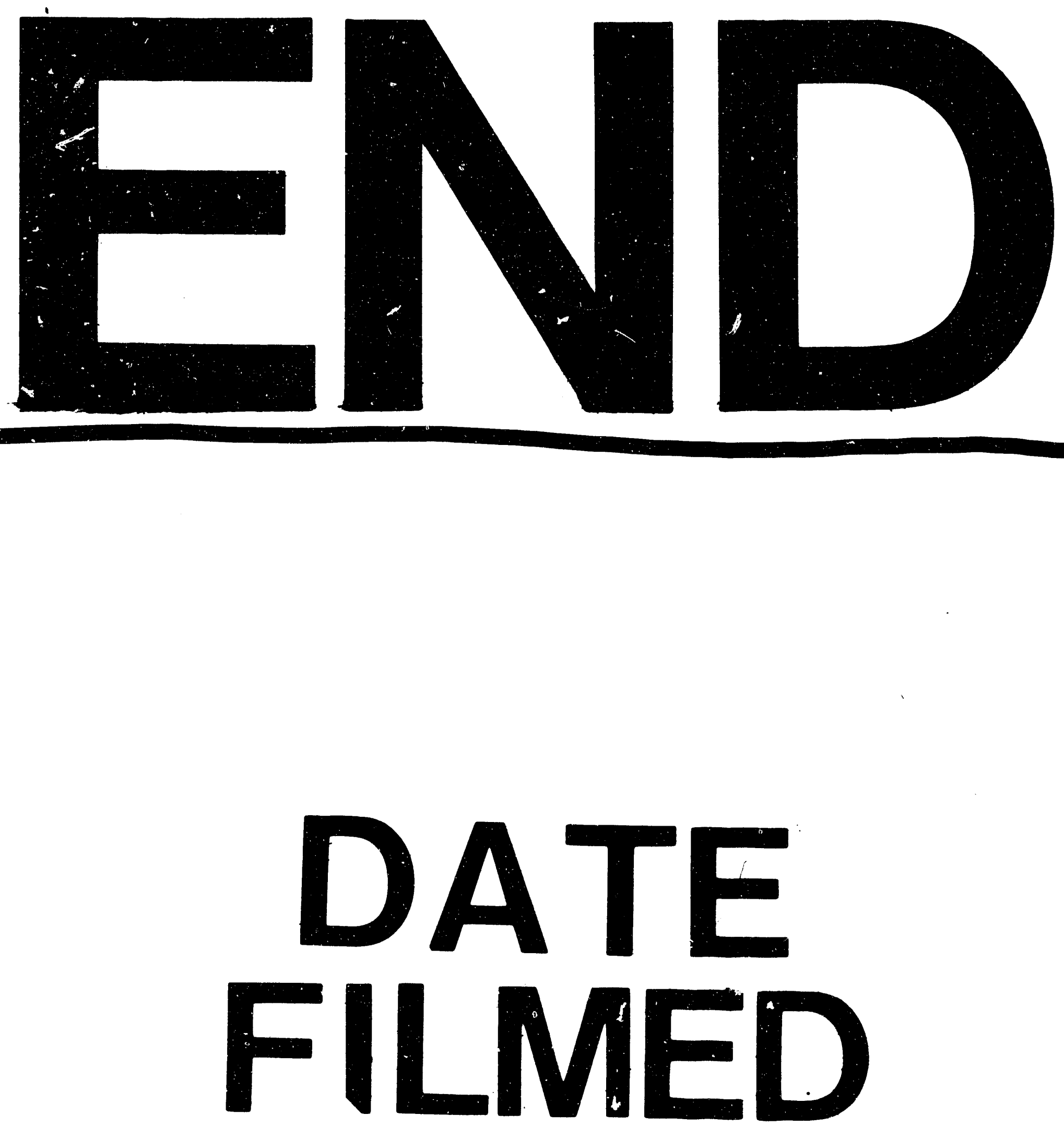

$=$
$=$
$=$
$=$
$=$
$\equiv$

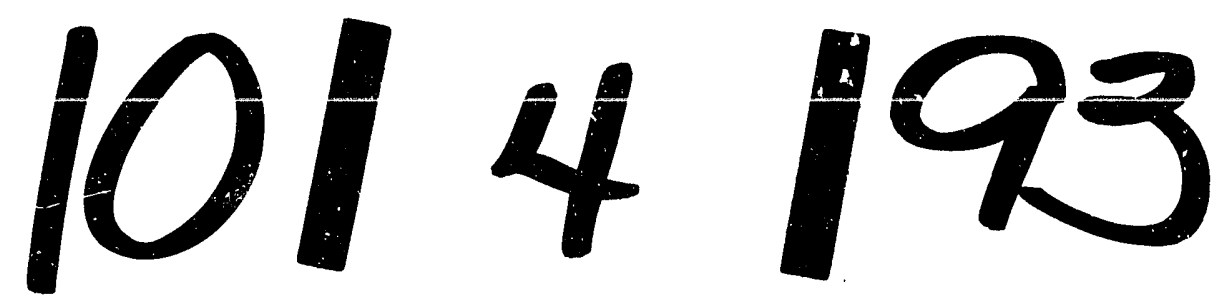


